\title{
Localized DNA Computation
}

\author{
by \\ Hieu Trung Bui \\ Department of Computer Science \\ Duke University \\ Date: \\ Approved: \\ John H. Reif, Supervisor \\ Alvin R. LeBeck \\ Raluca Gordan
}

Thomas H. LaBean

Dissertation submitted in partial fulfillment of the requirements for the degree of Doctor of Philosophy in the Department of Computer Science in the Graduate School of Duke University 


\section{ABSTRACT \\ Localized DNA Computation}

by

Hieu Trung Bui

Department of Computer Science

Duke University

Date:

Approved:

John H. Reif, Supervisor

Alvin R. LeBeck

Raluca Gordan

Thomas H. LaBean

An abstract of a dissertation submitted in partial fulfillment of the requirements for the degree of Doctor of Philosophy in the Department of Computer Science in the Graduate School of Duke University 
Copyright $@ 2017$ by Hieu Trung Bui

All rights reversed except the rights granted by the Creative Commons Attribution-Noncommercial License 


\section{Abstract}

Recently, solution-based systems for DNA computation have demonstrated the enormous potential of DNA nanosystems to do computation at the molecular-scale. These use DNA strands to encode values and use DNA hybridization reactions to perform computations. But most of these prior DNA computation systems relied on the diffusion of DNA strands to transport values during computations. During diffusion, DNA molecules randomly collide and interact in a three-dimensional fluidic space. At low concentrations and temperatures, diffusion can be quite slow and could impede the kinetics of these systems whereas at higher concentrations and temperature, unintended spurious interactions during diffusion can hinder the computations. Hence, increasing the concentration of DNA strands to speed up DNA hybridization reactions has the unfortunate side effect of increasing leaks, which are undesired hybridization reactions in the absence of input strands. Also, diffusion-based systems possess global states encoded via concentration of various species and hence exhibit only limited parallel ability.

To address these challenges, this dissertation describes a novel design for DNA computation called a localized hybridization network, where diffusion of 
DNA strands does not occur. Instead all of the DNA strands are localized by attaching them to an addressable substrate such as DNA nanotrack and DNA origami. This localization increases the relative concentration of the reacting DNA strands thereby speeding up the kinetics. This dissertation demonstrated a localized hybridization network that executed a chain reaction of five DNA hybridizations which executes faster than non-localized DNA reactions.

Another advantage of this approach is that each copy of the localized hybridization network operates independently of each other, allowing for a high level of parallelism. Localized hybridization networks also allow one to reuse the same DNA sequence to perform different actions at distinct location on the addressable substrate, increasing the scalability of such systems by exploiting the limited sequence space. An advantage of localized hybridization computational circuit is sharper switching behavior as information is encoded over the state of a single molecule. This also eliminates the need for thresholding as computation is performed locally eliminating the need for a global consensus.

There are many applications for localized hybridization networks. These include counting the number of disease marker molecules in a patient, detecting various cancer DNA sequences, and detecting and distinguishing bacteria by 
their distinguishing DNA. The results from localized DNA hybridization reactions may also be of practical use in performing surface computation on cellular membranes for disease detection and prevention. 


\section{Dedication}

To Melissa and Abigail. 


\section{Contents}

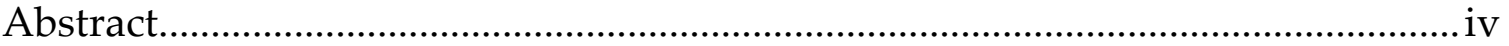

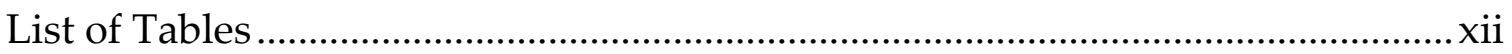

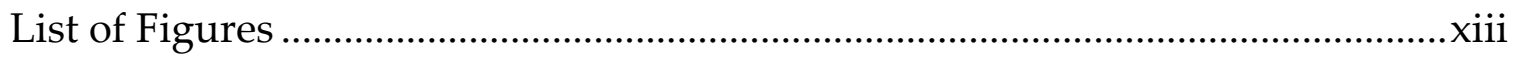

Acknowledgments ...............................................................................................

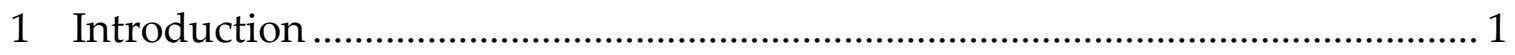

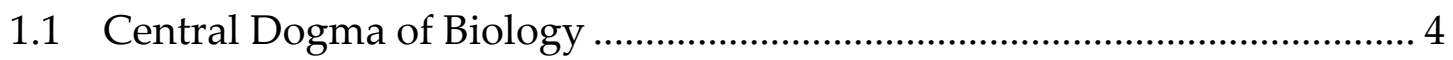

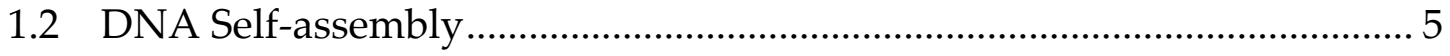

1.3 Structural DNA Nanotechnology .......................................................... 7

1.3.1 DNA Crossover Motifs......................................................................... 8

1.3.2 DNA Tiles and DNA Origami.......................................................... 9

1.4 Dynamic DNA Nanotechnology …………............................................... 11

1.4.1 DNA Hybridization Reactions ............................................................ 11

1.4.2 DNA Strand Displacement ................................................................ 12

1.4.3 Toehold and Toehold-Exchange Strand Displacements ...................... 12

1.4.4 DNA Gates: Duplex, Seesaw, Hairpin ……………................................. 15

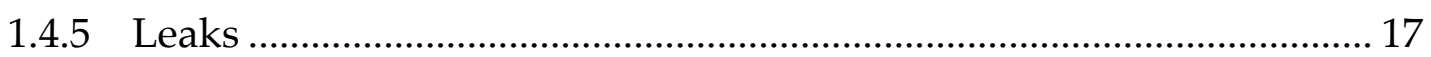

1.4.6 Mismatches, Spacers, Clamps and G-C contents ................................. 18

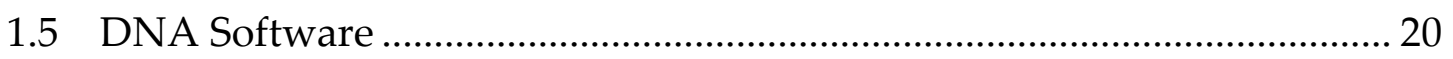

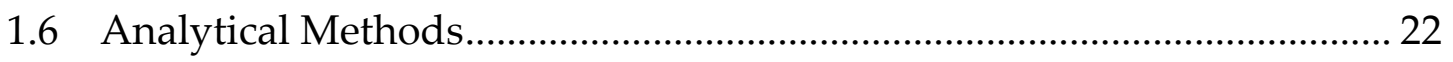




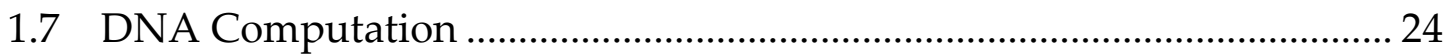

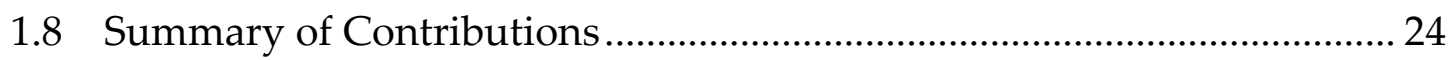

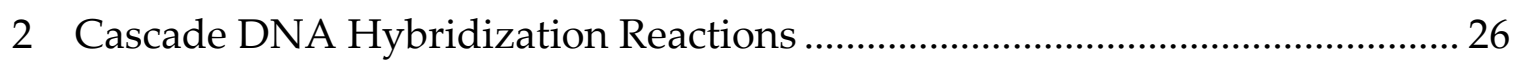

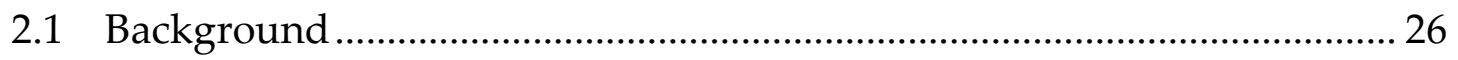

2.2 Mechanism of Cascade Chain Reaction ................................................... 28

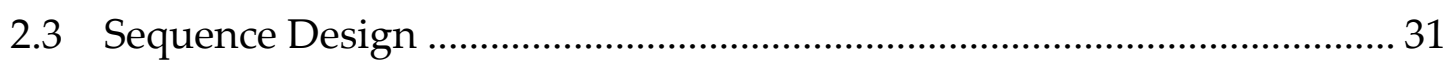

2.4 Chemical Reaction Networks for LCR .......................................................... 34

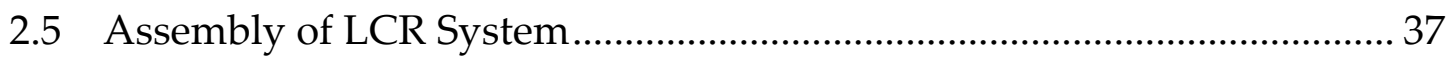

2.6 Thermal Equilibrium Characterization of LCR ......................................... 40

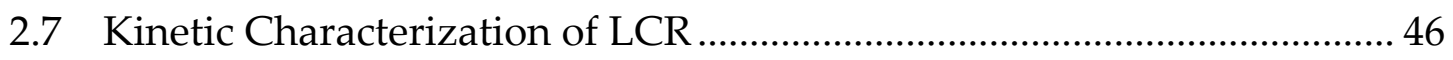

2.8 Model Validation and Synthesis Yield …………....................................... 51

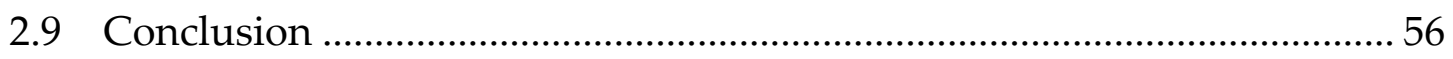

3 Localized DNA Hybridization Chain Reactions on DNA Nanostructures..... 57

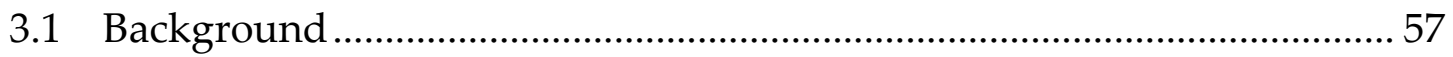

3.2 Localized DNA Hybridization Reactions.................................................... 58

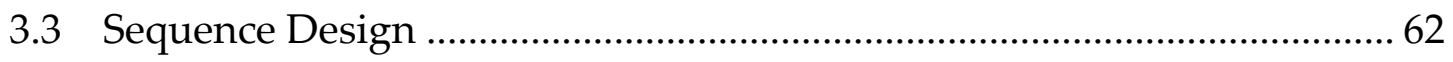

3.4 Mechanism of Localized DNA Hybridization Reactions............................ 64

3.5 Gel Electrophoresis Method and Ensemble Fluorescence Spectroscopy 65

3.6 Thermal Equilibrium Characterization......................................................... 67

3.7 Real Time Kinetic Characterization......................................................... 72 
3.8 Speed-up from tethering DNA hairpins to the surface

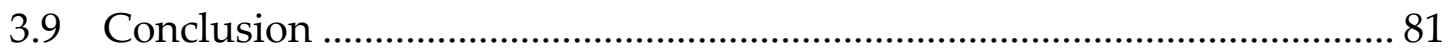

4 Localized DNA Hybridization Chain Reactions on Large DNA

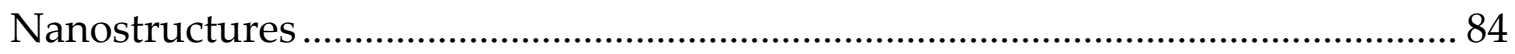

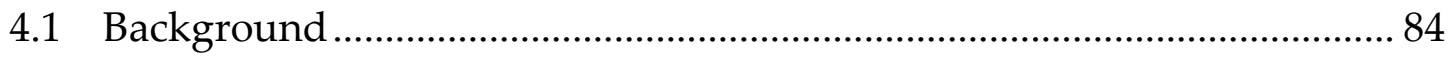

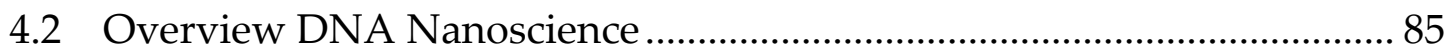

4.3 Design and mechanism of localized DNA hybridization chain reaction

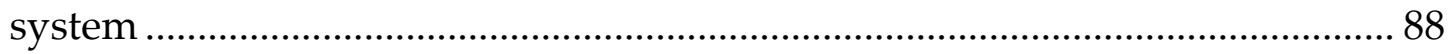

4.4 Assembly of DNA Origami Rectangle........................................................ 91

4.5 Atomic Force Microscopy Imaging Technique............................................ 93

4.6 Total Internal Reflection Fluorescence Microscopy ……………………..... 94

4.7 Ensemble Fluorescence Spectroscopy …………..........................................96

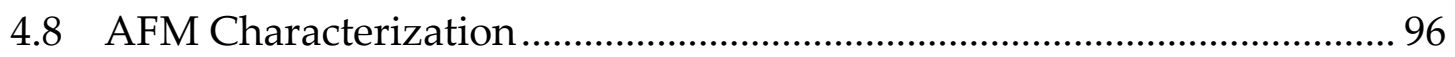

4.9 TIRFM Characterization............................................................................. 98

4.10 Ensemble Fluorescence Characterization .................................................. 106

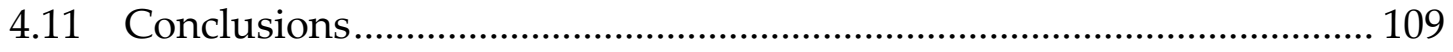

5 Localized DNA Computation for Constructing Molecular-Scale Synthetic

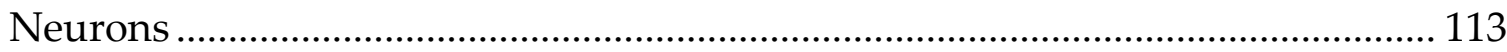

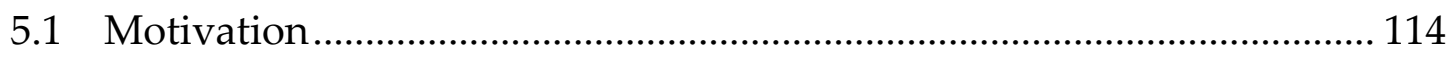

5.1.1 Prior Work in DNA Structural Nanoscience: DNA Nanostructures 115

5.1.2 Molecular Scale Imaging of DNA Nanostructures .............................. 116

5.1.3 Prior Work in Excitable Medium and Synthetic Neurons .................. 126 
5.2.1 Error Correction Strategies ................................................................... 131

5.2.2 Reversible Strategies............................................................................ 132

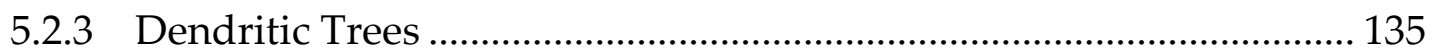

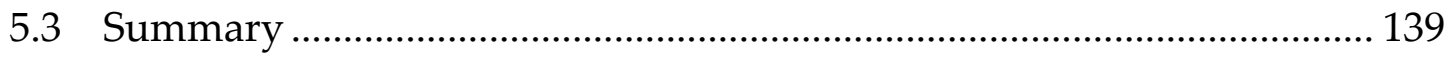

Appendix A - Scripts and DNA Sequences ........................................................... 140

Appendix B - Determination of Streptavidin-Biotin Binding Efficiency ……...... 153

Appendix C - Sequence Design for Constructing Redundancy and Reversible

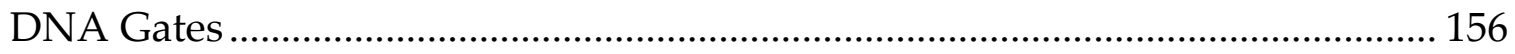

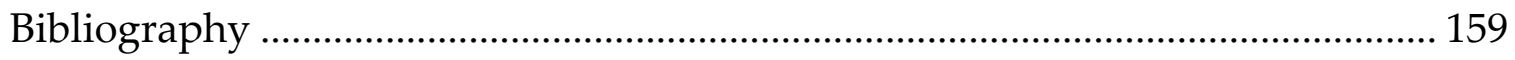

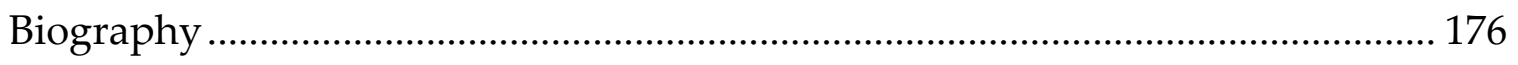




\section{List of Tables}

Table 2.1: DNA oligomers for constructing linear cascade DNA hybridization chain reactions. Note: toehold domains (colored green), stem domains (colored blue), clamp domains (colored orange), spacer domains (colored black). In hairpin sequences, stems are underlined. For kinetic studies, RC complex is displaced via the highlighted domain on hairpin $\mathrm{H}_{9}$.

Table 3.1: DNA oligomers to assemble linear cascade DNA hybridization chain reactions on the long DNA track. In hairpin sequences, stems are underlined. For kinetic studies, the reporter complex (RC) is displaced hairpin $\mathrm{H}_{6}$.

Table 4.1: DNA sequences for tethering DNA origami to the glass's surface via biotin-streptavidin conjugation.

Table 4.2: DNA oligomers for constructing the proposed system. For kinetic studies, RC duplex is displayed by hairpin $\mathrm{H} 6$. 111

Table A.1: A list of staple strands to form DNA origami rectangle. 145 


\section{List of Figures}

Figure 1.1: (A) Primary nucleotides for forming RNA and DNA. (B) WatsonCrick base pairing. (C) DNA double helix (B-form) with the periodicity of $10.5 \mathrm{bp}$ per turn and with the diameter of 20 angstroms..................................................... 6

Figure 1.2: Bottom-up approach to design structural DNA nanoscale objects and shapes. Beginning with the DNA double helix, DNA nanostructures can be constructed using different DNA crossover motifs.................................................... 8

Figure 1.3: Toehold-mediated DNA strand displacement mechanism. The toehold domain is colored green. The forward rate constant for $5 \mathrm{nt}$ (or longer) toehold domain is roughly from $10^{5}$ to $10^{6} \mathrm{M}^{-1} \mathrm{~S}^{-1}$. The free energy of the duplex is computed using NUPACK.

Figure 1.4: Toehold exchange DNA strand displacement mechanism. The toehold domain is colored green. The number of base pairing does not change.

Figure 1.5: Identification between DNA duplex and DNA hairpin molecules. For constructing non-equilibrium DNA systems, DNA molecules are programmed with either external toehold domain (colored green) or internal toehold domain (colored blue)

Figure 1.6: Introducing mismatch and spacer to control DNA hybridization reactions. Mismatch and spacer modifications are colored red. 19

Figure 2.1: (A) Components of the linear cascade DNA hybridization chain reaction system. (B) Autonomous reaction of controlled self-assembly. Hairpins 1 to 9 are stable in the absence of initiator I. The initiator binds at the sticky end of $\mathrm{H}_{1}$ and undergoes an unbiased strand displacement interaction to open the hairpin. The newly exposed sequestered sticky end of $\mathrm{H}_{1}$ binds to the external sticky end of $\mathrm{H}_{2}$ and opens the hairpin to expose a sequestered sticky end on $\mathrm{H}_{2}$. This cascade reaction continues until $\mathrm{H}_{9}$ is opened. The designed product is a linear chain formed by staggered hybridization of nine hairpins. 30 
Figure 2.2: Simulation results of various linear cascade reaction lengths. The length depends on the number of hairpins involved in each reaction. Linear cascade reactions with different rate constants $5 \times 10^{4} \mathrm{M}^{-1} \mathrm{~s}^{-1}(\mathrm{~A}), 10^{5} \mathrm{M}^{-1} \mathrm{~s}^{-1}(\mathrm{~B}), 5 \mathrm{x}$ $10^{5} \mathrm{M}^{-1} \mathrm{~s}^{-1}(\mathrm{C})$, and $1.3 \times 10^{6} \mathrm{M}^{-1} \mathrm{~s}^{-1}(\mathrm{D})$. Simulation is performed based on the assumption that all hairpin-hairpin pairs follow the second order (bimolecular) chemical reaction. Simulation conditions are specified as the following: $5 \mathrm{nM}$ concentration of hairpins, $6.5 \mathrm{nM}$ concentration of reporter complex, $10 \mathrm{nM}$ concentration of initiator, and the rate constant of displacing the reporter complex is common across all simulation experiments. 37

Figure 2.3: Native PAGE analysis of LCR: All samples were incubated at room temperature for at least 15 hours. (A) Effect of initiator concentration on LCR. All samples were prepared at $500 \mathrm{nM}$ and $20 \mu \mathrm{L}$ of sample was loaded into each lane. Lane 1: DNA marker with 20-bp increments $(20-300 \mathrm{bp})$; Lane 2: $0 \mu \mathrm{M}$ of initiator and $0.5 \mu \mathrm{M}$ mixture of $\mathrm{H} 1$ to $\mathrm{H} 9$ without overnight incubation. Lanes 3 to 8: six different concentrations of initiator $(0,0.5,1,2,4,8 \mu \mathrm{M})$ in a $0.5 \mu \mathrm{M}$ mixture of $\mathrm{H}_{1}$ to $\mathrm{H}_{9}$. (B) Step-by-step of the assembly of cascade chain reaction. The initiator was added $1 \mathrm{x}$. All samples were prepared at $100 \mathrm{nM}$ and $50 \mu \mathrm{L}$ of sample was loaded into each lane. Subsequent higher gel bands correspond to longer linear duplexes being formed. The largest linear duplex is resulted from the assembly of linear cascade DNA hybridization of 9 distinct hairpins. Lane 1: $\mathrm{H}_{1}+$ target DNA initiator; Lane 2: $\mathrm{H}_{1}+\mathrm{H}_{2}+$ target DNA initiator; Lane 3: $\mathrm{H}_{1}+\mathrm{H}_{2}$ $+\mathrm{H}_{3}+$ target DNA initiator; Lane $4: \mathrm{H}_{1}+\mathrm{H}_{2}+\mathrm{H}_{3}+\mathrm{H}_{4}+$ target DNA initiator; Lane 5: $\mathrm{H}_{1}+\mathrm{H}_{2}+\mathrm{H}_{3}+\mathrm{H}_{4}+\mathrm{H}_{5}+$ target DNA initiator; Lane 6: $\mathrm{H}_{1}+\mathrm{H}_{2}+\mathrm{H}_{3}+\mathrm{H}_{4}+\mathrm{H}_{5}+$ $\mathrm{H}_{6}+$ target DNA initiator; Lane 7: $\mathrm{H}_{1}+\mathrm{H}_{2}+\mathrm{H}_{3}+\mathrm{H}_{4}+\mathrm{H}_{5}+\mathrm{H}_{6}+\mathrm{H}_{7}+$ target DNA initiator; Lane 8: $\mathrm{H}_{1}+\mathrm{H}_{2}+\mathrm{H}_{3}+\mathrm{H}_{4}+\mathrm{H}_{5}+\mathrm{H}_{6}+\mathrm{H}_{7}+\mathrm{H}_{8}+$ target DNA initiator; Lane 9: $\mathrm{H}_{1}+\mathrm{H}_{2}+\mathrm{H}_{3}+\mathrm{H}_{4}+\mathrm{H}_{5}+\mathrm{H}_{6}+\mathrm{H}_{7}+\mathrm{H}_{8}+\mathrm{H}_{9}+$ target DNA initiator; Lane 10: DNA marker with 20-bp increments (20-300 bp).

Figure 2.4: Native PAGE gel of different linear cascade reactions involving 2 hairpins, 4 hairpins, and 6 hairpins with and without addition of target DNA initiator. (Gel A) Lane 1: DNA marker with 10-bp increments (10 - $150 \mathrm{bp);} \mathrm{Lane}$ 2: mixture of $\mathrm{H}_{8}$ and $\mathrm{H}_{9}$; Lane 3: annealing of $\mathrm{H}_{8}$ and $\mathrm{H}_{9}$; Lane $4: \mathrm{H}_{8}+$ target DNA initiator; Lane 5: $\mathrm{H}_{8}+\mathrm{H}_{9}+$ target DNA initiator. (Gel B) Lane 1: DNA marker with 20-bp increments (20 - $300 \mathrm{bp}$ ); Lane 2: mixture of $\mathrm{H}_{6}$ and $\mathrm{H}_{7}$; Lane 3: mixture of $\mathrm{H}_{6}, \mathrm{H}_{7}$, and $\mathrm{H}_{8}$; Lane 4: mixture of $\mathrm{H}_{6}, \mathrm{H}_{7}, \mathrm{H}_{8}$, and $\mathrm{H}_{9}$; Lane 5: $\mathrm{H}_{6}+$ 
target DNA initiator; Lane 6: $\mathrm{H}_{6}+\mathrm{H}_{7}+$ target DNA initiator; Lane 7: $\mathrm{H}_{6}+\mathrm{H}_{7}+\mathrm{H}_{8}$ + target DNA initiator; Lane 8: $\mathrm{H}_{6}+\mathrm{H}_{7}+\mathrm{H}_{8}+\mathrm{H}_{9}+$ target DNA initiator. (Gel C) Lane 1: DNA marker with 20-bp increments (20-300 bp); Lane 2: mixture of $\mathrm{H}_{4}$ and $\mathrm{H}_{5}$; Lane 3: mixture of $\mathrm{H}_{4}, \mathrm{H}_{5}$, and $\mathrm{H}_{6}$; Lane 4: mixture of $\mathrm{H}_{4}, \mathrm{H}_{5}, \mathrm{H}_{6}$, and $\mathrm{H}_{7}$; Lane 5: mixture of $\mathrm{H}_{4}, \mathrm{H}_{5}, \mathrm{H}_{6}, \mathrm{H}_{7}$, and $\mathrm{H}_{8}$; Lane 6: mixture of $\mathrm{H}_{4}, \mathrm{H}_{5}, \mathrm{H}_{6}, \mathrm{H}_{7}, \mathrm{H}_{8}$, and $\mathrm{H}_{9}$; Lane 7: $\mathrm{H}_{4}+$ target DNA initiator; Lane 8: $\mathrm{H}_{4}+\mathrm{H}_{5}+$ target DNA initiator; Lane 9: $\mathrm{H}_{4}+\mathrm{H}_{5}+\mathrm{H}_{6}+\mathrm{H}_{7}+$ target DNA initiator; Lane 10: $\mathrm{H}_{4}+\mathrm{H}_{5}+\mathrm{H}_{6}+\mathrm{H}_{7}+\mathrm{H}_{8}+$ target DNA initiator; Lane 11: $\mathrm{H}_{4}+\mathrm{H}_{5}+\mathrm{H}_{6}+\mathrm{H}_{7}+\mathrm{H}_{8}+\mathrm{H}_{9}+$ target DNA initiator.

Figure 2.5: Kinetic characterization of the linear cascade DNA hybridization chain reactions. All samples were prepared at $5 \mathrm{nM}$ of hairpin with $6.5 \mathrm{nM}$ of reporter complex. (A) Schematic of LCR system before and after interacting with the reporter complex. (B) Effect of initiator concentration on the rate of linear cascade reaction. Hairpins do not hybridize in the absence of initiator (9 hairpins $+0 \mathrm{x}$ I, black). The linear cascade reaction occurs in the presence of $1 \mathrm{x}$ initiator concentration (9 hairpins $+1 x \mathrm{I}$, red). The linear cascade reaction occurs at the same rate in the presence of excess initiator (9 hairpins + 8x I, green). (C) Effect of hairpin concentration on the rate of crosstalk and leak reactions: $5 \mathrm{nM}$ (black curve), $25 \mathrm{nM}$ (red curve), and $50 \mathrm{nM}$ (green curve) without the presence of initiator.

Figure 2.6: (A) Effect of the number of hairpins on the rate of linear cascade reaction: 1 hairpin (black curve), 2 hairpins (red curve), 4 hairpins (green curve), 6 hairpins (blue curve), 9 hairpins (cyan curve). Note: the dotted horizontal line indicates the half-life of the linear cascade reaction (the time required for the reaction to reach $50 \%$ completion). Inset shows the real-time kinetic of all linear cascade reactions at equilibrium. (B) Empirical projection of the half-time completion as a function of hairpins. Note: each circle is an experimental result corresponding to the number of hairpins participating in the linear cascade reaction; a blue line is a fitted curve from those circles. 51

Figure 2.7: Fitting the experimental results using the proposed model. Scatter plots are experimental data; Line curves are fitted data using the least squares method. 53 
Figure 2.8: Estimation of yield via maximum fluorescence intensity readout. All experimental data were collected at $5 \mathrm{nM}$ concentration. Assume that the maximum fluorescence intensity at thermal equilibrium is correlated with the yield of hairpins participating in the linear cascade reaction. Black curve is the fluorescence emission of initiating the last hairpin and resulting in displacing the reporter complex (schematic B). Cyan curve is the fluorescence emission of initiating the first hairpin and resulting in displacing the reporter complex after the linear cascade reaction (schematic $\mathrm{C}$ ). The maximum fluorescence at thermal equilibrium of the linear cascade consisting of a single hairpin is 72 a.u. The maximum fluorescence at thermal equilibrium of the linear cascade consisting of 9 distinct hairpins is 57 a.u. To the first order approximation, the yield of 9 hairpins is approximated $79 \%$ 55

Figure 3.1: Depiction of localized DNA hybridization chain reactions on DNA nanotrack. The presence of the surface enables the reaction to proceed faster.... 58

Figure 3.2: (A) Components of localized DNA hybridization chain reaction system: six metastable DNA hairpins anchored to a long DNA track. Prior to the addition of the initiator, DNA hairpins do not hybridize. (B) Triggered selfassembly of localized DNA hybridization chain reaction mechanism for the first and last steps. (C) Fluorescence reporting mechanism to observe the kinetics of localized DNA hybridization reaction. 61

Figure 3.3: Intermediate states of the localized DNA hybridization chain reaction system. After initiation (top-left), the reaction reaches the completion when all hairpins are hybridized (top-right).

Figure 3.4: (Left panel) 10\% native PAGE gel analysis of HCR: All samples were incubated at room temperature for 200 minutes. All samples were prepared at $500 \mathrm{nM}$ and $34 \mu \mathrm{L}$ of sample was loaded into each lane. All hairpins do not carry the anchor domains. Lane 1 contains DNA marker with 20-bp increments (20$300 \mathrm{bp}$ ). Lanes 2 to 7 contain mixture of different numbers of hairpins in the absence of the initiator. Lanes 8 to 13 contain the same corresponding mixture of hairpins in the presence of the initiator. (Middle and right panels) 3\% agarose gel analysis of localized DNA hybridization chain reaction (LCR). All hairpins are conjugated with the anchor domains to self-assemble to the long DNA track. All 
samples were quickly annealed for 10 minutes and incubated with the initiator for 200 minutes at room temperature. All samples were prepared at $500 \mathrm{nM}$ and $10 \mu \mathrm{L}$ of sample was loaded into each lane. (Middle panel) Step-by-step LCR $500 \mathrm{nM}$ of hairpins were mixed with $2 \mathrm{x}$ LT in the presence of $2 \mathrm{x}$ initiators (I). (Right panel) Effect of initiator concentration to LCR - $500 \mathrm{nM}$ of hairpins were mixed with $2 x$ LT in the presence of the initiator. Note that the marker maroon boxes indicate major bands of the desired DNA structures. .70

Figure 3.5: Kinetic characterization of triggered self-assembly hybridization chain reactions. (A) Localized DNA hybridization chain reactions with and without the presence of the long DNA track in 2 times excess initiators. Note: inset shows the fluorescence responses of the same systems but in the absence of the initiator. (B) Effect of localized DNA hybridization chain reaction rate as a function of initiator concentration. (C) Control kinetic analysis of reactions as a function of hairpins in the absence of long DNA track.

Figure 3.6: Reproducible fluorescence experimental results of the two systems which are then used to quantify the speed-up from tethering DNA hairpins on the long DNA track: (A) six DNA hairpins (no anchor domain, no track) in the presence of 2x initiators, (B) six DNA hairpins bound to the long DNA track in the presence of $2 x$ initiators. Note: Dashed lines indicate half-time completion the time required for the reaction to reach $50 \%$ completion. (C) Model to describe cascade reactions with and without the locality effect. Note: AND symbol indicates bimolecular reaction; TRIANGLE symbol indicates unimolecular reaction.

Figure 4.1: Summary of localized DNA hybridization chain reaction. (A) DNA origami rectangle with modified staple strands (green circles). Metastable DNA hairpins tethering to DNA origami rectangle's surface via modified staple strands through the anchor domains. (B) Mechanism of DNA hybridization reactions on DNA origami surface.

91

Figure 4.2: DNA origami rectangle design. Staple strands are colored red. Scaffold strand is colored black. Modified staple strands for tethering DNA hairpins are colored green. Biotin-labeled staple strands are colored purple. Modified staple strand for attaching marker sequence is colored blue. .93 
Figure 4.3: AFM characterization of DNA origami rectangle labeled with DNA hairpins at thermal equilibrium. Six metastable DNA hairpins self-assembled on DNA origami rectangle (A), after adding the initiator (B), and after adding the biotin-labeled output to detect the reaction completion (C). Additional data are shown in Figure 4.4 with bigger survey areas. 98

Figure 4.4: AFM characterization of DNA origami rectangle labeled with DNA hairpins at thermal equilibrium at $2 \mu \mathrm{m}$ scan size. Six metastable DNA hairpins self-assembled on DNA origami rectangle (A), after adding the initiator (B), and after adding the biotin-labeled output to detect the reaction completion (C)..... 98

Figure 4.5: Total internal reflection fluorescence characterization (TIRF). TIRF farfield microscopy image of solution containing DNA hairpins on origami before initiation (A), during reaction (B) and after completion (C). Left column is the fluorescence emission of the dye-labeled strand after being excited with a designated excitation wavelength. Right column is the schematic of the dyelabeled strand after being excited with a designated excitation wavelength. All the scale bars are $1 \mu \mathrm{m}$. Additional data are shown in Figure 4.6 with larger survey areas. 100

Figure 4.6: Kinetics of localized cascade DNA hybridization reactions overtime via TIRFM. Left column: fluorescence emission of the dye-labeled marker strand(A, D, G, J). Middle column: fluorescence emission of the dye-labeled initiator strand (B, E, H, K). Right column: fluorescence emission of the dyelabeled output strand (C, F, I, L). Scale bars are $1 \mu \mathrm{m}$........................................... 101

Figure 4.7: Visualizing localized DNA hybridization chain reaction via TIRF. Fluorescence emission of detecting the reaction completion overtime (10 (A), 70 (B), and 200 (C) minutes). Number of detected spots increased as thermal equilibrium was reached (D). All scale bars are $1 \mu \mathrm{m}$ and these images are superresolved with uncertainty between 10 and $20 \mathrm{~nm}$. Refer to Figure 4.8 for additional data. 104

Figure 4.8: Super-resolved data after exciting the dye-labeled output strand while monitoring the emission overtime: (A) 10 minutes, (B) 70 minutes, and (C) 200 minutes. Scale bars are $5 \mu \mathrm{m}$............................................................................. 106 
Figure 4.9: Ensemble fluorescence characterization. (A) Schematic of localized cascade DNA hybridization chain reaction on the surface of DNA origami rectangle before and after the completion. (B) Kinetic characterization of localized cascade DNA hybridization chain reaction on DNA origami rectangle (green curve). Kinetic characterization of cascade DNA hybridization chain reaction without DNA origami rectangle (purple curve). Each solution was kept at $5 \mathrm{nM}$ concentration and the initiator was added in $2 x$ excess. (C) Effect of localized cascade DNA hybridization chain reaction rate as a function of initiator concentration. 107

Figure 5.1: Using redundancy (via branching) to increase chain reaction success. Each gate is encoded with 2 identical outputs. After initiation, the gate $G_{i}$ produces two identical outputs. Either of the outputs can trigger the next gate $\mathrm{G}_{\mathrm{i}+1}$.

Figure 5.2: Reversible using toehold-mediate DNA hairpins. By controlling the DNA hybridization base pairing, the system is designed to revert back to the original state.

Figure 5.3: An architecture to construct error correction and branching tree. Using redundancy and branching to increase chain reaction success and to reset chain reaction. After initiation, the gate $\left(\mathrm{G}_{\mathrm{i}}\right)$ can hybridize to either the adjacent downstream gates $\left(\mathrm{G}_{\mathrm{i}+1}\right)$. After forward reactions, a set of destabilizing gates is introduced to delaminate the gates from the prior reactions. A complement set of removal hairpins is introduced to pull back DNA strands and allows gates to reconfigure back to the initial state. A detail of designing DNA sequences for the above strategies is presented in Appendix C. 134

Figure 5.4: Using redundancy and resetting gates to construct dendritic trees. Actual implemented gates are indicated in dashed rectangle boxes. (A) A single branch of two linear chains, (B) A dendritic binary tree, and (C) Two dendritic trees T1 and T2 with the common root allowing for multiple inputs and multiple outputs, and (D) The cyclic patterning of activation signals is initiated once and is governed by the localized reactions. Note: each node is represented by a desired gate. 137 
Figure B.1: DNA origami rectangles labeled with biotinylated oligomers: (A) 5 nM DNA rectangle sample without streptavidin, (B) 5 nM DNA rectangle sample with $443 \mathrm{nM}$ streptavidin, (C) $5 \mathrm{nM}$ DNA rectangle sample with $121 \mathrm{nM}$ streptavidin, (D) $5 \mathrm{nM}$ DNA rectangle sample with $55 \mathrm{nM}$ streptavidin, (E) $5 \mathrm{nM}$ DNA rectangle sample with $15 \mathrm{nM}$ streptavidin. 154

Figure B.2: Binding yield between biotin and streptavidin on the surface of DNA origami rectangle.

Figure B.3: Binding efficiency between biotin and streptavidin molecules. Maximum available spots for binding (A) 3 molecules and (B) 1 molecule. ...... 155

Figure C.1: Programming reversible DNA hybridization reactions using DNA hairpins. The design consists of a pair of DNA hairpin gates that can go forward and reverse. In particular, the presence of the initiator triggers the forward reaction and the presence of the destabilizing and removing hairpins trigger the reverse reaction. Note: the length of each domain can be found in Script C.1... 158 


\section{Acknowledgments}

This dissertation could not have been completed without the help and support from many individuals. First I would like to thank my advisor, John Reif, for his guidance and support throughout this process. I'm extremely grateful to have an opportunity to work with him. Second I would like to thank my committee members: Alvy LeBeck, Thomas LaBean, and Raluca Gordan for their commitments and supports. I would like to thank Robert Duvall, Susan Rodger, and Owen Astrachan for inspiring me in teaching with countless hours of grading exams during weekends and making teaching fun. I would like to thank my research lab members, in particular, Sudhanshu Garg, Reem Mokhtar, and Tianqi Song for being there for me throughout this journey. We shared a lot of fond memories that were filled with both social activities and intellectual discussion. Other Reif's group members for making group meetings interesting and engaging: Shalin Shah, Abeer Eshra, Xin Song, Dan Fu. Past members Mingxin Lu, Vincent Miao, Amy Yang, Mike Zhu, Tong Niu, Jeff Du, and Brian Petkov.

My friends and colleagues at Duke: Rachael Al-Saadon, Mauricio PiloPais, Anne Watson, Craig LaBoda, Marcel Frenkel, John MacMaster, Abhishek 
Dubey, and Risi Thonangi. Cohorts and friends from CS department: Ben Stoddard, Nadi Bozkurt, Ali Razeen, Ergys Ristani, Mayuresh Kunjir, Animesh Srivastava, Xi He, Ios Kotsogiannis, Jiangwei Pan, Branka Lakic, Austin Alexander, Yuzhang Han, Garrett Andersen, Pablo Gainza and Yezhou Huang. Thanks to Marilyn Butler for everything you have done to make my graduate school going smoothly and effectively. Thanks to Camelia Eaves for your tremendous help in organizing FNANO conference annually. Thanks to Pam Spencer for your help since the first day I came to Duke. Thanks to Melanie Eberhart for assisting in making purchases seem to be an easy job. Thanks to Joe Shamlin and Jeff Wright for proving excellent IT supports office and laboratory equipment upgrades.

Thanks to my family (mom, dad, Hien Bui, Hoa Bui, Hoang Bui, Hau Bui, and Hang Bui) for letting me make my own decision and always be there for me. Thanks to my wife, Trang Le, for your support, companionship, and love with our two beautiful daughters: Melissa and Abigail. 


\section{Introduction}

Living organisms are an example of self-replicating systems that can evolve. In order to understand the assembly of these systems, we need to be able to create artificial systems with similar capabilities. Inspired from biomolecular motors in living cells, many ingenious synthetic molecular motors have been created. Given the complexity of the biological organisms, these synthetic molecular motors are still not capable of carrying out evolution in vitro. To assist in developing these synthetic molecular motors for useful applications, DNA computation and other forms of molecular computation have been used. In particular, the molecular and supramolecular interactions can be mimicked through applying fast computational algorithms.

Recently, solution-based systems for DNA computation $^{1}$ have demonstrated the enormous potential of DNA nanosystems to do computation at the molecular-scale. ${ }^{2}$ These use DNA strands to encode values and use DNA hybridization reactions to perform computations ${ }^{3}$. But most of these prior DNA computation systems relied on the diffusion of DNA strands to transport values during computations. During diffusion, DNA molecules randomly collide and interact in a three-dimensional fluidic space. At low concentrations and 
temperatures, diffusion can be quite slow and could impede the kinetics of these systems. At higher concentrations and temperature, unintended spurious interactions occur and hinder the computations. Hence, increasing the concentration of DNA strands to speed up DNA hybridization reactions has the unfortunate side effect of increasing leaks, which are undesired hybridization reactions in the absence of input strands ${ }^{4}$. Also, diffusion-based systems possess global states encoded via concentration of various species and hence exhibit only limited parallel capability.

To address these challenges, this research described in this dissertation makes use of a novel design for DNA computation called a localized hybridization network ${ }^{5}$, where diffusion of DNA strands does not occur. In contrast, all prior attempts to do localized DNA computation have required some diffusion of DNA strands during intermediate stages of the computations. Instead all the DNA strands are localized by attaching them to an addressable substrate such as DNA nanotrack and DNA origami. This localization increases the relative concentration of the reacting DNA strands thereby speeding up the kinetics. This study demonstrated a localized hybridization network that 
executed a chain reaction of five DNA hybridizations which executes faster than non-localized DNA reactions ${ }^{6}$.

Another advantage of this technique is that each copy of the localized hybridization network operates independently of each other, allowing for a high level of parallelism. Localized hybridization networks also allow one to reuse the same DNA sequence to perform different actions at distinct location on the addressable substrate, increasing the scalability of such systems by exploiting the limited sequence space. An advantage of localized hybridization computational circuit is sharper switching behavior as information is encoded over state of a single molecule. This also eliminates the need for thresholding as computation is performed locally eliminating the need for a global consensus ${ }^{4}$.

There are many applications for localized hybridization networks. These include counting the number of disease marker molecules in a patient, detecting various cancer DNA sequences, and detecting and distinguishing bacteria by their distinguishing $\mathrm{DNA}^{7-9}$. The results from localized DNA hybridization reactions may also be of practical use in performing surface computation on cellular membranes for disease detection and prevention. 


\subsection{Central Dogma of Biology}

Nucleic acids, proteins, lipids, and carbohydrates are the main building blocks of biological systems. Nucleic acids consist of two distinct types which are ribose nucleic acids (RNA) and deoxyribose nucleic acids (DNA). In eukaryote cells, DNA molecules reside inside the nucleus and unravel during the transcription process to make RNA molecules. On the other hand, RNA molecules are responsible for transporting the transcribed DNA information beyond the nucleus. The translation process, making proteins from RNA, takes place outside the nucleus but within the lipid cell membranes. In contrast, prokaryote cells lack a nucleus and all of the reproduction processes (i.e. the transcription and translation) occurs in a similar way as of those in eukaryote cells. Understanding the biological systems broadly requires multidisciplinary expertise. In an attempt to further our understanding of the biological systems, this dissertation attempts to focus and investigate the property and behavior of DNA hybridization reactions on two-dimensional surfaces. Other biological building blocks are concurrently being explored by other researchers and are not the focus of this dissertation. For a complete overview, Francis Crick reported a detailed explanation of multiple transfer pathways with the most currently understanding of the biological systems ${ }^{10}$. 


\subsection{DNA Self-assembly}

RNA, DNA, peptides, proteins, and enzymes are the foundation of biological diversity. Beyond conventional properties of DNA, scientists have been using DNA for constructing nanoscale structures, smart materials, and circuits. The ability to precisely control DNA hybridization reactions in vitro opens up opportunities to explore plethora applications in bioscience fields as well as in engineering disciplines. DNA is often referred to as "the blueprint of life" because it contains all the information needed to create and sustain life. Most naturally occurring DNA exists in the double helix form with the diameter of roughly 20 angstroms and the periodicity of 10.5 base pairs (bp) per turn (equivalent to $3.4 \mathrm{~nm}$ ). In biological environment, DNA often interacts with enzymes to produce functional molecules such as RNA, peptides, and proteins. Unlike natural DNA, synthetic DNA or oligonucleotide or oligomer can be synthesized and exist in either single-stranded DNA (ssDNA) or doublestranded DNA (dsDNA). A DNA hybridization occurs when adenine (A) nucleotide binds with thymine $(\mathrm{T})$ nucleotide, and guanine $(\mathrm{G})$ nucleotide binds with cytosine (C) nucleotide. These binding events are often called the WatsonCrick base pairing. Other unconventional binding events could occur such as Hoogsteen base pairing or Wobble base pairing. Throughout this document, 
DNA sequences are designed based on the following assumptions: 1) DNA strands strictly follow Watson-Crick base pairs, 2) the duplex or double helix is strictly B-form with $10.5 \mathrm{bp}$ per turn and 2-nm diameter, and 3) unconventional binding events do not take place. In addition, the DNA systems are constrained mostly with DNA with no involvement of RNA, amino acids, peptides, or proteins.

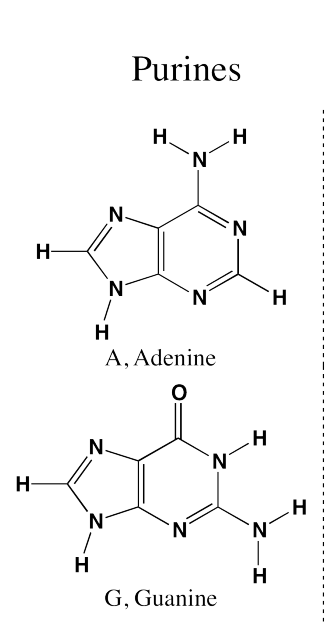

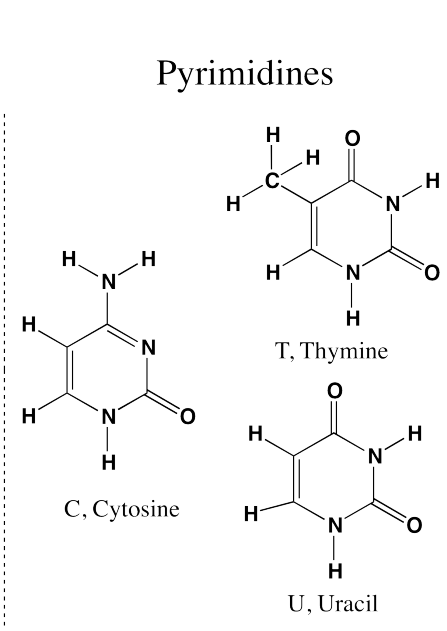

(A)

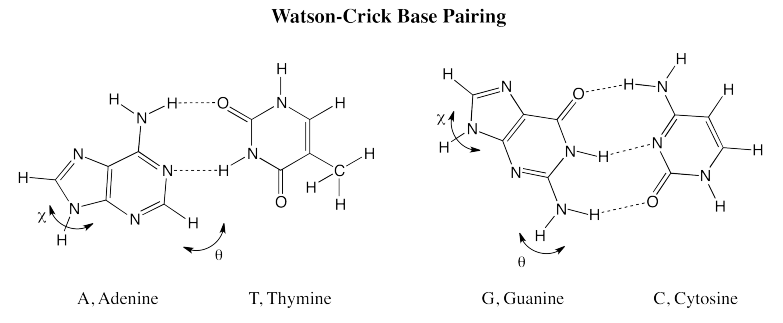

(B)

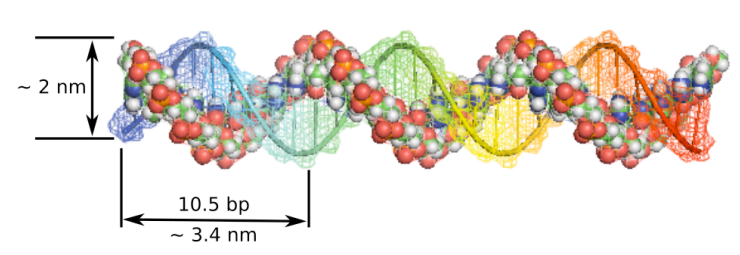

(C)

Figure 1.1: (A) Primary nucleotides for forming RNA and DNA. (B) WatsonCrick base pairing. (C) DNA double helix (B-form) with the periodicity of $10.5 \mathrm{bp}$ per turn and with the diameter of 20 angstroms.

Conventional DNA Parameters: Assuming B-form DNA double helix throughout this document, thus it has 10.5 base pairs per turn. One base pair length is 
equivalent to $0.34 \mathrm{~nm}$ and each base pair is twisted from the previous one by $34^{\circ}$. Due to the double helix formation, the geometry of the base pair gives rise to the two uneven grooves (i.e. minor and major). Other DNA forms exist such as Aform and Z-form with different helical turns as well as other characteristics.

\subsection{Structural DNA Nanotechnology}

Utilizing Watson-Crick DNA hybridization, any arbitrary nanoscale objects can be designed, synthesized, and constructed using techniques offering from the field of structural DNA nanotechnology ${ }^{11-13}$. The first example of structural DNA objects was demonstrated by Ned Seeman ${ }^{14}$ when he successfully created DNA lattices from hybridizing pre-programmed DNA sequences together. DNA origami ${ }^{15}$, DNA brick ${ }^{16}$ and ssDNA tile ${ }^{17}$ techniques were later introduced and provided additional capabilities to create $2 \mathrm{D}$ and 3D DNA nanostructures. Although most shapes and objects can be created, the final nanostructures are often lack of functionalities. To create functional DNA nanostructures, researchers often organized other molecular components ${ }^{18-21}$ to those structures. To understand the mechanism of forming DNA nanostructures, the following subsections will briefly introduce a few key concepts. 

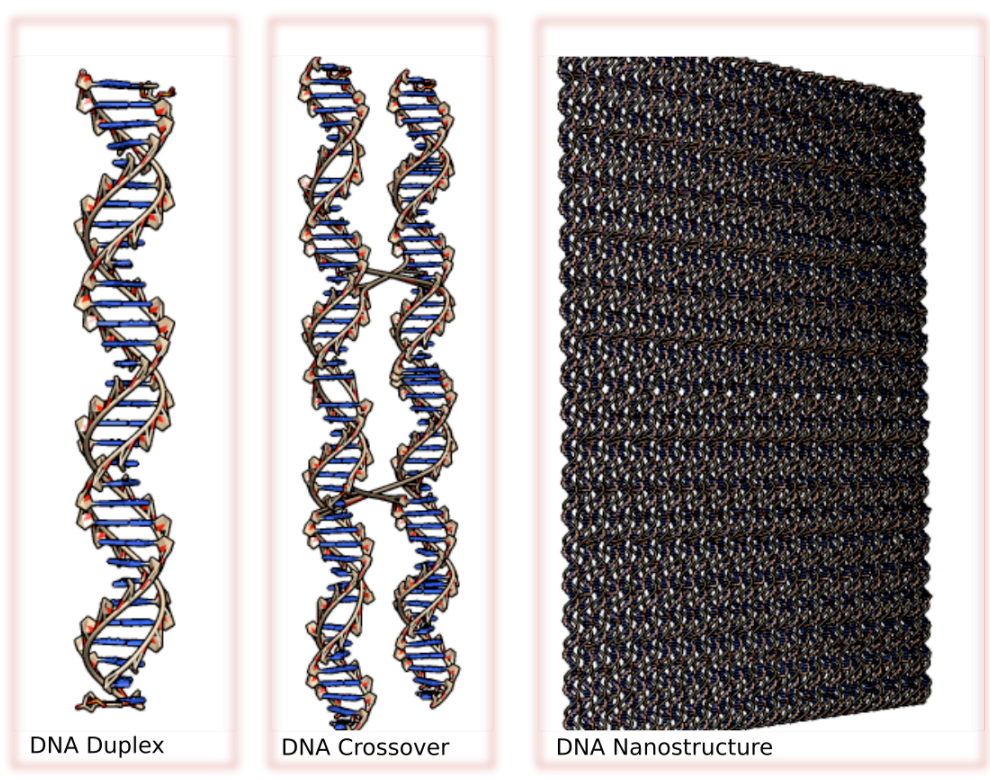

Figure 1.2: Bottom-up approach to design structural DNA nanoscale objects and shapes. Beginning with the DNA double helix, DNA nanostructures can be constructed using different DNA crossover motifs.

\subsubsection{DNA Crossover Motifs}

Double Crossover: A double crossover ${ }^{22}$ (DX) is a basic building motif of DNA nanostructures. The DX consists of two double helical domains joined twice at crossover points. There are five unique DX motifs. For instance, DPE is a double crossover that is separated by an even number of half-turns of DNA and parallel. DPOW is a double crossover that is separated by an odd number of half-turns of DNA, parallel and the extra half-turn is a major groove spacing. DPON is a double crossover that is separated by an odd number of half-turns of DNA, parallel, and the extra half-turn is a minor groove spacing. DAE is an even 
double crossover. DAO is an odd double crossover. Anti-double crossover and parallel-double crossover are difference in the directionality of DNA motifs. The location of the crossover is often determined based on the length and shape of the final nanostructure. To wrap a DNA strand to form a 90-degree turn, the crossover is designed to be a multiple of 8 nucleotides. Similarly to form a 120degree turn, the crossover is designed to be a multiple 7 nucleotides.

Triple Crossover: A triple crossover ${ }^{23}$ is similar to the double crossover with an exception of an additional crossover as suggested by the name. This motif enables the flexibility to connect more DNA strands together. It's worth noting that the tensile strength of this motif may be different than that of the double crossover. Other crossover motifs such as G-quadruplex or i-motif, can be introduced to create a diverse set of branched DNA nanostructures. The persistent length of DNA duplex is around $50 \mathrm{~nm}(100 \mathrm{bp})^{11}$. Majority of DNA nanostructures can be designed using these crossover motifs ranging from simple honeycomb DNA lattices ${ }^{24}$ to three-dimensional DNA cuboids ${ }^{16}$.

\subsubsection{DNA Tiles and DNA Origami}

To form large DNA nanostructures from the crossover motifs, different folding concepts have been proposed. For example, DNA tiles are often 
constructed from a finite set of DNA strands utilizing various DNA crossover motifs as mentioned previously. Experimentally it is difficult to achieve high yield due to i) the difficulty of maintaining the stoichiometry among the strands and ii) the difficulty of controlling the self-assembly process via the thermal anneal process. The largest nanostructure is the hexagonal 2D arrays which were reported to extend to micrometer in length ${ }^{24}$.

DNA origami technique uses a long scaffold DNA and a large set of distinct short staple strands. The long scaffold is first folded visually into a final shape. Subsequently short strands are designed to bind complementary to the long scaffold via Watson-Crick hybridization. The short staple strands are then generated with the help from computer-aid software such as CaDNAno ${ }^{25}$. To validate the formation of designed nanostructures, all short staple strands are mixed with the long scaffold strand in 5:1 ratio and subjected through one-pot annealing process. Scanning probe microscopy is often used to confirm $2 \mathrm{D}$ and 3D DNA origami nanostructures. Depend on the need, either DNA tiles or DNA origami nanostructures can be employed to serve as the surface to attaching active DNA components for localization studies. 


\subsection{Dynamic DNA Nanotechnology}

Understanding the basic rules that govern DNA hybridization reactions, one can manipulate DNA strands to be programmed to form the functional materials. In particular, researchers have created non-equilibrium DNA systems that can behave like switches, robots, and circuits. In order to understand the mechanism of these devices, the following section will introduce several key concepts that are responsible for majority of the discussed DNA systems.

\subsubsection{DNA Hybridization Reactions}

A DNA hybridization reaction occurs when two single-stranded DNA sequences are complementarity to each other and self-assemble to form a stable DNA duplex based on the Watson-Crick hybridization principle. In the early 2000s, Yurke et al. demonstrated the first engineered DNA hybridization reaction via the construction of DNA tweezers ${ }^{26}$. Afterwards, Zhang et al. refined the technique and built several models based on the toehold mediated strand displacement $^{27}$. The latest progress in understanding the DNA strand displacement process has been pushed forward by Srinivas et $\mathrm{al}^{28}$. In particular, they articulated that there are at least four distinct time scales that govern the toehold-mediate strand displacement. It now seems reasonable to develop more 
complex nanodevices that could be used as scientific instruments or as diagnostic tools.

\subsubsection{DNA Strand Displacement}

DNA strand displacement (DSD) is a process which alters the thermal equilibrium condition of non-equilibrium DNA systems by introducing additional DNA molecules. In particular, DNA duplex molecules are often designed with non-hybridized single-stranded DNA molecules which are often called toeholds. Upon introducing other DNA molecules that often bind to the toeholds as well as to the remaining nucleotides to form new DNA duplex molecules, the non-equilibrium DNA systems undergo conformational changes to a lower more stable energy state. SantaLucia presented a comprehensive model to capture the entire thermodynamics of DNA-DNA interactions ${ }^{29}$.

\subsubsection{Toehold and Toehold-Exchange Strand Displacements}

A toehold is an un-hybridized single-stranded DNA strand as briefly mentioned in previous section. The toehold length ranges from 2 to 10 nucleotides (nt). The toehold's length affects the DNA hybridization rate. A short toehold ( $<3 \mathrm{nt})$ can quickly bind to a complementary strand but the binding strength is often weak at room temperature. A long toehold (> $10 \mathrm{nt})$ yields 
insignificant changes in terms of the reaction kinetics. A typical rate constant for toehold strand displacement is roughly $10^{6} \mathrm{M}^{-1} \mathrm{~s}^{-1}$ and the toehold strand displacement process is followed a classic bimolecular reaction. The process often drives the chemical reaction from a high energy state to a lower energy state. The number of base pairing within the systems after the strand displacement process often increases. The proposed DNA systems in this dissertation mainly utilize the toehold strand displacement process. 


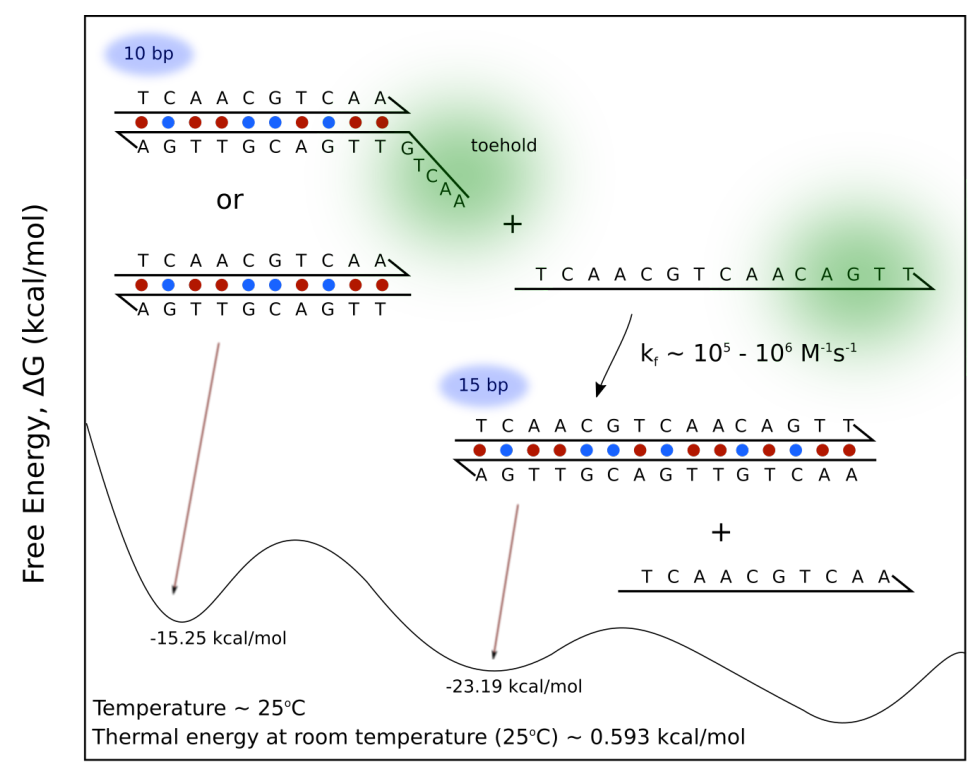

DNA duplex (bp)

Figure 1.3: Toehold-mediated DNA strand displacement mechanism. The toehold domain is colored green. The forward rate constant for $5 \mathrm{nt}$ (or longer) toehold domain is roughly from $10^{5}$ to $10^{6} \mathrm{M}^{-1} \mathrm{~s}^{-1}$. The free energy of the duplex is computed using NUPACK.

However, other DNA systems can be designed such that there is insignificant change in free energy by employing the toehold-exchange strand displacement concept. Zhang et al. reported a detail analysis of DNA systems utilizing the toehold-exchange idea ${ }^{30}$. The number of base pairing within the systems before and after the strand displacement process remains unchanged. The toehold exchange mechanism is used to continuously expose the downstream reaction with a new toehold. Since the reaction can proceed in bidirectional, the systems have the tendencies to fluctuate and other strategies 
have to be implemented to achieve desired functionalities. DNA seesaw systems are a representative example of utilizing the toehold exchange concept ${ }^{4}$.

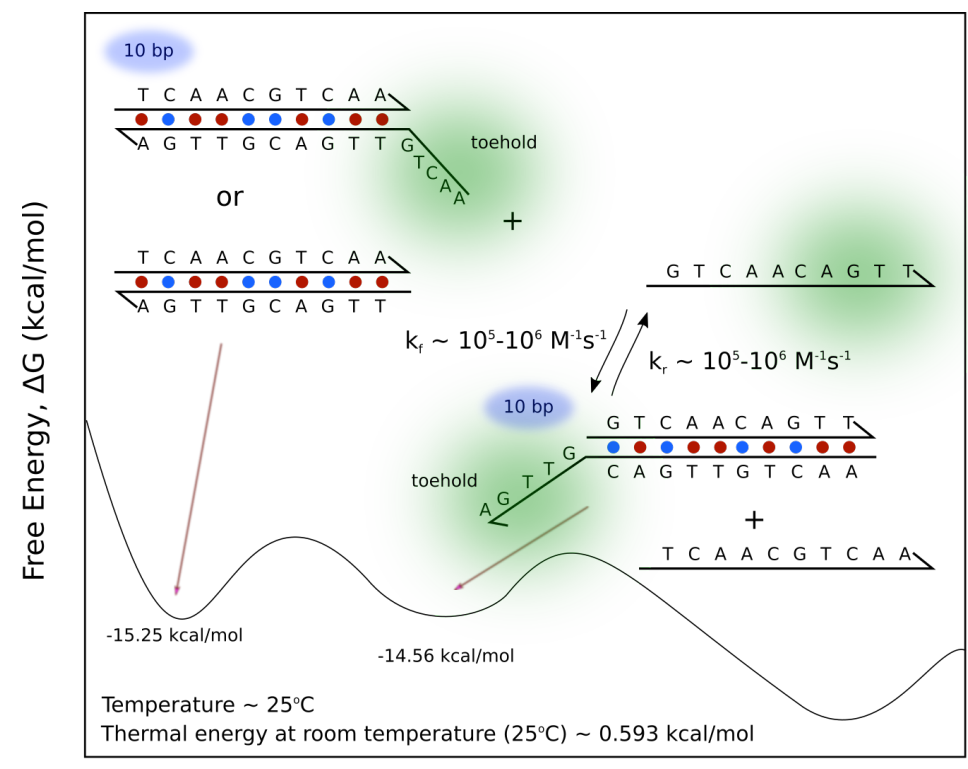

DNA duplex (bp)

Figure 1.4: Toehold exchange DNA strand displacement mechanism. The toehold domain is colored green. The number of base pairing does not change.

\subsubsection{DNA Gates: Duplex, Seesaw, Hairpin}

The next step to design DNA systems is to introduce different architecture to build and represent gates. A straight forward gate consists of a DNA duplex with either a toehold or a toehold-exchange. Another type of gate consists of a DNA hairpin with either a toehold or a toehold-exchange. In term of toehold design, the hairpin structure enables two possible locations to incorporate them. One is located at the stem (external toehold) and the other is located within the loop (internal toehold) ${ }^{31}$. Since the internal toehold presents steric hindrance to 
the binding events, the reaction rate which relies on the internal toehold tends to be slower than that of the external toehold. To compensate for this disadvantage, researchers tend to utilize longer internal toehold domains to go with short external toehold domains to maintain relatively similar kinetics. Cooperative hybridization ${ }^{32}$, remote toehold hybridization ${ }^{33}$, allosteric hybridization ${ }^{34,35}$ techniques were later introduced to provide additional capabilities for constructing DNA gates. DNA seesaw gates are often designed to be bi-stable and there is a constant competing between the input and the output strands to occupy the substrate. A threshold concept can be utilized to bias the reaction pathways either to the forward or reverse direction. For future generation of localized DNA computation, DNA gates may need to be designed in a similar approach as DNA seesaw gates to drive the forward and reverse reactions, thus activatable and reusability can be achieved for additional functionalities. 


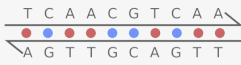

DNA Duplex

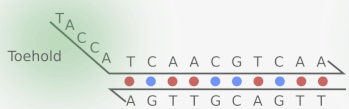

DNA Duplex
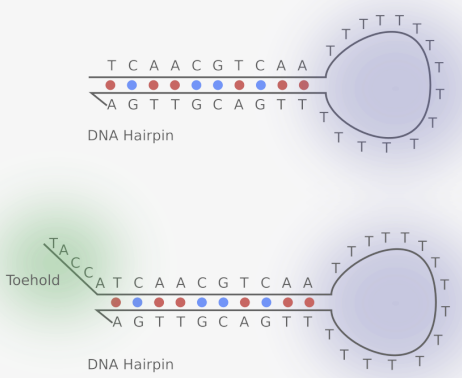

Figure 1.5: Identification between DNA duplex and DNA hairpin molecules. For constructing non-equilibrium DNA systems, DNA molecules are programmed with either external toehold domain (colored green) or internal toehold domain (colored blue) .

\subsubsection{Leaks}

One of the major challenges in designing non-equilibrium DNA systems is spurious interactions, crosstalk, or leaks. A leak is defined as the triggering of unintended DNA strand displacement reactions. A leak is an example of unfavorable DNA reactions and is not captured during modeling and simulation processes. Leaks are imminent in the actual experiments. There are several strategies to suppress leaks: i) designing sequences to have strong G-C bonds to minimize of fraying, ii) introducing clamp domains of 1-3 nucleotides at both ends of helices, iii) introducing Watson-Crick mismatches, iv) segregating different DNA complexes through iterative gel electrophoresis, v) introducing threshold gates to consume undesired strands, and vi) introducing long binding 
domains. However, there is no universal approach to combat leaks in DNA systems and researchers have to choose a right strategy for their systems. The following section briefly describe a few key concepts to address leaks.

\subsubsection{Mismatches, Spacers, Clamps and G-C contents}

Mismatches can be introduced to DNA gates to slow down the reaction kinetics $^{36}$. In addition, mismatches can be incorporated at the ends of DNA duplex to reduce the breathing effect, thus leaks can be minimized. Jiang et al. reported 100-fold increased signal-to-noise ratios from testing their catalytic hairpin assembly. 

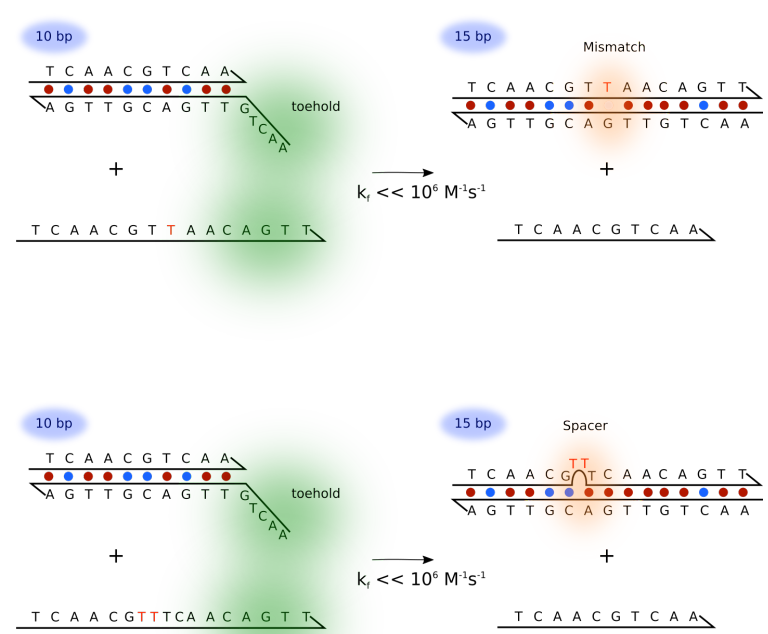

Figure 1.6: Introducing mismatch and spacer to control DNA hybridization reactions. Mismatch and spacer modifications are colored red.

Spacers are non-hybridize nucleotides that are embedded within DNA gates. Genot et al. introduced spacers between the toehold and displacement domains of both the substrate and invading strand $\mathrm{s}^{33}$ and were able to control the strand displacement rate by over 3 orders of magnitudes through the spacer domains. Another alternative to minimize the breathing effect at both ends of DNA gates is to introduce clamps which are often to be two to four base pairs ${ }^{37}$. G-C contents have been utilized to alter the reaction kinetics in DNA systems. In particular, guanine tends to bind strongly with cytosine; however, it can interact with other bases which prevents the reaction kinetics to proceed in the intended trajectory. Recently, scientists have proposed domain-level motifs for the design 
of leak-resistant DNA strand displacement systems ${ }^{38}$. For the proposed systems, designated strategies to combat leaks will be addressed in more details in the subsequent chapters.

\subsection{DNA Software}

To assist in programming DNA sequences either for constructing DNA nanostructures or non-equilibrium DNA systems, several useful software have been created such as $\mathrm{Mfold}^{39}$, NUPACK ${ }^{40}$, OxDNA ${ }^{41}, \mathrm{DSD}^{42,43}$, $\mathrm{caDNAnO}^{25}$, CanDo ${ }^{44}$, etc. The following will briefly highlight the usage and limitation of some key software.

Mfold $^{39}$ is an application to predict the secondary structure of single stranded nucleic acids both RNA and DNA. A similar software to Mfold is NUPACK ${ }^{40}$ which is a programming software for the analysis and design of nucleic acid structures and systems. NUPACK has additional features to design DNA systems consisting of more than two individual strands and to optimize the sequences with minimal defects or crosstalk. Recently OxDNA ${ }^{41}$ has been introduced and is a programming software for designing, simulating, and visualizing DNA circuits implementing the coarse-grained DNA model. OxDNA has been used to model the formation of DNA duplexes from single strands 
along with insights into the operation of complex systems such as thermodynamic, structural and mechanical changes in both DNA and RNA.

To model the reaction kinetics of DNA systems, Language for Biological Systems (LBS) and DNA Strand Displacement (DSD) can be employed ${ }^{42}$. In particular, LBS is based on Calculus of Biochemical Systems (CBS) and a textual language. LBS provides modular descriptions of molecular networks in terms of reactions between modified complexes. LBS features are species expressions, parameterized modules and non-determinism. LBS allows modification site types for representing DNA sequences. Whereas DSD is a programing language for designing and simulating computational devices made of DNA. DSD uses DNA strand displacement as the main computational mechanism. Complex molecules such as DNA hairpins have recently been introduced, however these software do not capture the sequence-level interactions among DNA gates. In other words, DNA gates are abstractly treated as molecules and strictly follow designated chemical reactions.

To design DNA nanostructures, $\mathrm{caDNAno}^{25}$ is often employed. caDNAno is a prototype software for design 2D and 3D DNA structures based on DNA origami concept. The software has two built-in lattices (square and honeycomb). 
Other underutilized software such as Tiamat ${ }^{45}$, Uniquimer ${ }^{46}$, and SARSE $^{47}$ can be used to design large DNA nanostructures. To predict 3D structure of DNA origami designs, $\mathrm{CanDo}^{44}$ can be utilized. In particular, CanDo uses the finite element method which models DNA base pairs as two-node beam finite elements, to compute 3D DNA origami nanostructures based on caDNAno design and Tiamat design files. Several software will be used for the proposed systems and more details will be addressed in the subsequent chapters.

\subsection{Analytical Methods}

To validate the correctness of DNA systems, analytical methods are needed. The following section will introduce frequent key methods to characterize DNA systems in laboratory settings.

Atomic force microscopy (AFM) is a scanning probe microscopy to capture the topological changes of nanoscale materials via the control feedback loop systems. In particular, DNA nanostructures can be characterized in the liquid conditions as well as in aqueous conditions. AFM is a routine method to characterize the correctness of DNA objects. Although DNA double helix cannot be measured directly by AFM due to the physical limitation of the probes, conjugated DNA double helix with nanoparticles can be observed indirectly. 
Transmission electron microscopy (TEM) is utilized to observe DNA nanostructures with high resolution. Since DNA molecules are not conductive, they are often stained with radioactive materials to enhance the contrast while performing TEM imaging.

Gel electrophoresis is a versatile technique to separate DNA by size. Using polyacrylamide gel electrophoresis, individual DNA strands with about $20 \mathrm{bp}$ differences can be distinguished. Using agarose gel electrophoresis, bigger DNA molecules such as DNA origami can be separated from short DNA molecules such as staple strands. Gel electrophoresis is also employed to study the thermal equilibrium conditions of DNA systems.

Fluorescence spectroscopy is used to indirectly measure the reaction kinetics of DNA systems via dye-labeled DNA gates. Ensemble fluorescence spectroscopy measures the average reaction effect of DNA systems whereas single-molecule fluorescence spectroscopy measures individual responses of the same systems. The rate constants of non-equilibrium DNA systems are often determined from the fluorescence responses. Recently DNA PAINT method has been demonstrated to capture the reaction kinetics of DNA systems by combining total internal reflection microscope with built-in laser setup. 


\subsection{DNA Computation}

DNA molecules interact with a set of other species, called inputs, and based on interactions with each subset of inputs, produce or do not produce another species, called an output, as a 'decision'1. The rules of decision-making can be presented in the form of truth tables, similar to logical operations or Boolean circuits. Thus these molecules can be called molecular logic gates and groups of molecules can be viewed as circuits. In modern electronic computers, logic gates are basic units that perform computing. A NOT gate uses one input molecule and produces the output only if that input is not present. An AND gate uses two input molecules and only produces an output if both inputs are present. An OR gate uses two input molecules and an output is produced if either or both inputs are present. To build more complex circuits, these basic computing elements are employed. DNA molecular logic gates use oligonucleotides as inputs and generate new oligonucleotides as outputs based on a set of rules.

\subsection{Summary of Contributions}

The scientific contributions presented in this dissertation are summarized in the following: Chapter 2 introduces a systematic approach to design DNA sequences, to simulate DNA-DNA interactions using different software tools, 
and to validate the design via performing actual experiments. In particular, a non-equilibrium DNA system consists of 9 distinct DNA hairpin gates is investigated. Chapter 3 presents a novel construction of localized hybridization chain reactions on small DNA nanostructures. Using the same hairpin design previously, the benefit of localized DNA computation is demonstrated via tethering DNA hairpin gates to a long DNA track. Chapter 4 presents an advanced construction of the similar system but using large DNA nanostructures (i.e. DNA origami). Chapter 5 presents extended localized DNA computation designs to allow for branching and error-correction for the construction of molecular-scale neurons and future work. 


\section{Cascade DNA Hybridization Reactions}

Given the background information about DNA self-assembly, the advantage of programming DNA self-assembly rather than other biochemical molecules, and the benefits of constructing dynamic DNA hybridization systems, Chapter 2 demonstrates an implementation of constructing a simple DNA hybridization system combining the techniques within the field of DNA nanoscience. In this chapter, a simple linear cascade DNA hybridization chain reaction implementing DNA hairpins is demonstrated with the following key concepts: sequence design, DNA simulation, experimental implementation and modeling. This chapter was published as: Hieu Bui, Sudhanshu Garg, Vincent Miao, Tianqi Song, Reem Mokhtar and John Reif, “Design and analysis of linear cascade DNA hybridization chain reactions using DNA hairpins", New Journal of Physics 19 (2017). DOI: http://dx.doi.org/10.1088/1367-2630/aa53d0

\subsection{Background}

Deoxyribonucleic acids (DNA) are one of the major building blocks of biological systems. Recently, researchers have been exploiting DNA selfassembly for constructing nanostructures as well as programming molecular

devices $12,13,48-58$. Yurke et al. first observed the phenomenon of DNA strand 
displacement in their nanomachine ${ }^{26}$. Based on DNA strand displacement thermodynamics, the construction of molecular systems ${ }^{27}$, logic circuits ${ }^{4,59}$,

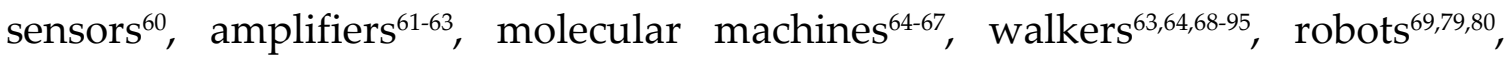
chemical controllers ${ }^{96}$, and neural networks ${ }^{97}$ were realized. The use of DNA hybridization as the primary driving energy source has been the central theme in many DNA systems. An alternative energy source could also come from metastable DNA ${ }^{98}$. For instance, Turberfield et al. used unhybridized DNA hairpin loops as the fuel source to operate DNA motors ${ }^{99}$. In addition, energy from DNA hairpins has been used to assist with catalytic hybridization ${ }^{31}$ and triggered self-assembly ${ }^{63,100}$. Inspired by early work on triggered self-assembly of DNA nanostructures ${ }^{63,100-102}$, we present a system for the controlled self-assembly of linear cascade DNA hybridization chain reactions (LCR) using DNA hairpins. Previous studies have demonstrated the formation of linear DNA oligomers from open hairpins hybridized in a staggered two-repeat monomer pattern 100,102, with or without the assistance of a few auxiliary strands. Yin et al. extended the triggered self-assembly from linear growth oligomers to quadratic growth branched oligomers and exponential growth dendritic oligomers ${ }^{63}$. Our system lies in the regime of linear growth oligomers with the following advantages: i) 
our system has 9 distinct hairpins which can potentially form a longer chain, whereas prior works ${ }^{100-103}$ used only two to four hairpins, ii) our system allows fluorescence detection at any given oligomer length, iii) our system enables quantification of chain reaction success rate for any given length, which can be tailored to design larger systems as and when required, iv) the original system by Dirks and Pierce ${ }^{100}$ had to be carefully tailored to have a stem of $18 \mathrm{nt}$ and a toehold/hairpin loop region of $6 \mathrm{nt}$. Our system however, introduces clamp domains, removing this constraint, thus allowing us to design systems with variable stem ( $>=12 \mathrm{nt})$ and toehold lengths, which considerably increases the sequence space and applicability of these systems and v) because our system has 9 distinct hairpins (rather than the 2 hairpins of Dirks's system ${ }^{100}$ ), it can be extended to do localized reactions on a surface ${ }^{104,105}$. Our experimental results indicate strong evidence of linear cascade reaction responses based on gel electrophoretic analysis and reaction kinetics.

\subsection{Mechanism of Cascade Chain Reaction}

The assembly linear cascade DNA hybridization chain reaction is shown in Figure 2.1. Figure 2.1A shows the components: nine hairpins $\left(\mathrm{H}_{1}, \mathrm{H}_{2}, \mathrm{H}_{3}, \mathrm{H}_{4}\right.$, $\mathrm{H}_{5}, \mathrm{H}_{6}, \mathrm{H}_{7}, \mathrm{H}_{8}, \mathrm{H}_{9}$ ), and an initiator (I). Each hairpin consists of a stem, a loop, 
and a sticky end. The stem consists of two domains $\left(C_{i}\right.$ and $\left.R\right)$ which form a stable duplex with 14 base pairs. The loop consists of two clamp domains ( $\mathrm{C}_{\mathrm{i}-1}$ and $\left.C_{i+1}\right)$, a spacer domain $(L)$, and a sequestered domain $\left(S_{i+1}\right)$. The sticky end consists of an external toehold domain $\left(\mathrm{S}_{\mathrm{i}}\right)$ which is complementary to the loop domain $\left(\mathrm{S}_{\mathrm{i}-1}\right)$ of hairpin $\left(\mathrm{H}_{\mathrm{i}-1}\right)$. Hairpins are distinguishable by two singlestranded toehold domains $\left(S_{\mathrm{i}}\right.$ and $\left.S_{\mathrm{i}+1}\right)$ - one toehold is external (a single-stranded sticky end, readily available for hybridization) and one is sequestered within the hairpin loop. The stem $(\mathrm{R})$ and spacer $(\mathrm{L})$ domains of all hairpins comprise the same sequences. The spacer domain (L) is used to offset potential geometrical constraints. The clamp domain (C) is implemented to minimize the breathing effect at the ends of the DNA duplex and to ensure that the cascade reaction proceeds in the forward direction ${ }^{106}$.

Initially, all hairpin components are mixed together but do not hybridize. The cascade reaction is initiated by the initiator (I) which interacts with $\mathrm{H}_{1}$ by hybridizing to the external toehold $\left(\mathrm{S}_{1}\right)$ as illustrated in Figure 1B. The initiator displaces the stem of $\mathrm{H}_{1}$ by branch migration, opening the loop and revealing its sequestered toehold domain $\left(\mathrm{S}_{2}\right)$. The opened loop of $\mathrm{H}_{1}$ can now bind to the external toehold of $\mathrm{H}_{2}$ and displace the stem of $\mathrm{H}_{2}$ by branch migration, opening 
in turn the loop of $\mathrm{H}_{2}$. This reaction cascades downstream as each additional hairpin anneals to the growing structure (which displaces the stem and opens the loop, making the previously sequestered toehold accessible for hybridization to initiate the next stage of chain growth). The designed product of the assembly is a linear chain formed by the staggered hybridization of nine hairpins. A reporter complex was used to determine the rate of the chemical reaction. Hairpin $\mathrm{H}_{9}$ triggered the opening of the reporter complex, as shown in Figure 2.1A.

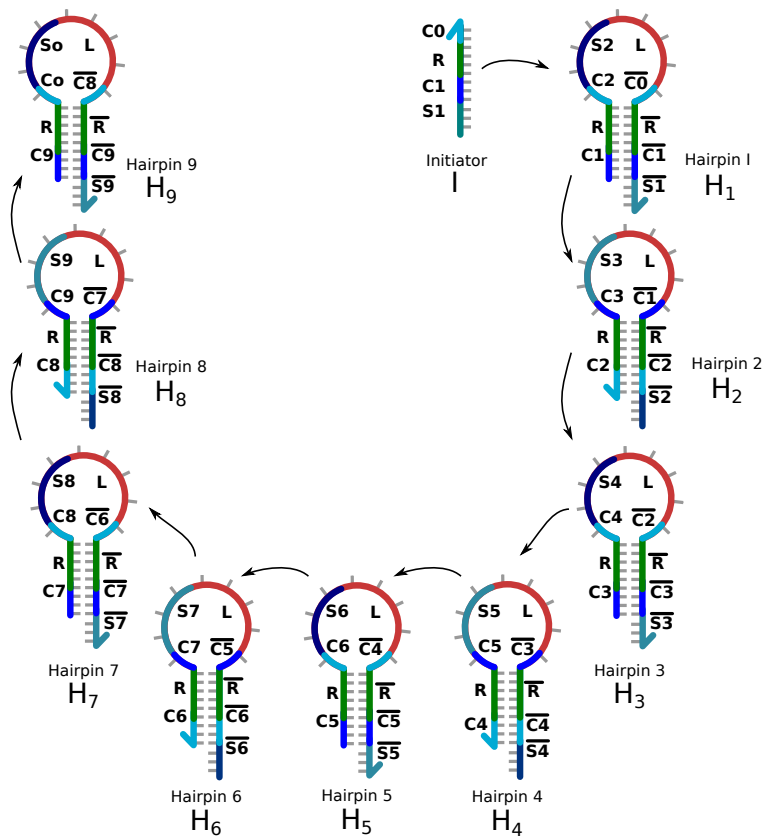

(A)

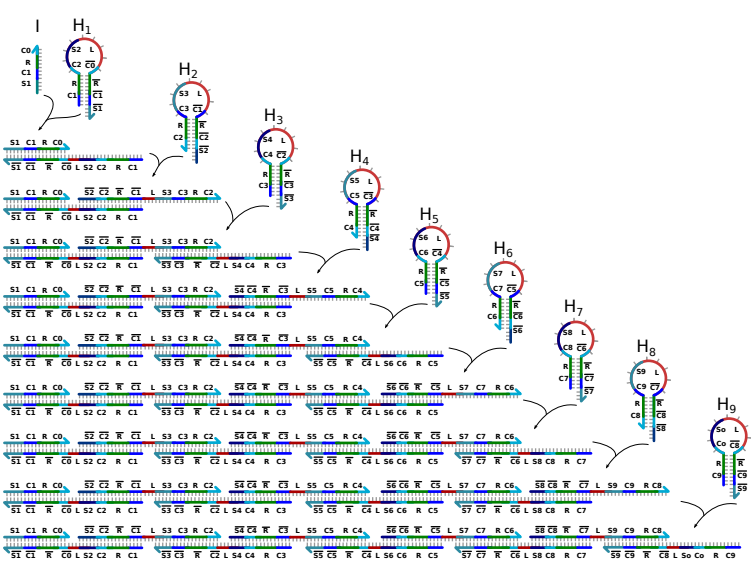

(B)

Figure 2.1: (A) Components of the linear cascade DNA hybridization chain reaction system. (B) Autonomous reaction of controlled self-assembly. Hairpins 1 to 9 are stable in the absence of initiator I. The initiator binds at the sticky end of $\mathrm{H}_{1}$ and undergoes an unbiased strand displacement interaction to open the hairpin. The newly exposed sequestered sticky end of $\mathrm{H}_{1}$ binds to the external 
sticky end of $\mathrm{H}_{2}$ and opens the hairpin to expose a sequestered sticky end on $\mathrm{H}_{2}$. This cascade reaction continues until $\mathrm{H}_{9}$ is opened. The designed product is a linear chain formed by staggered hybridization of nine hairpins.

\subsection{Sequence Design}

In general, the design process begins with a set of target DNA nanostructures consisting of $n$ hairpins and initiators. Each hairpin structure has a 14-bp stem, 18-nt loop, and 6-nt external toehold. Hairpin $n$th has a slightly longer loop length of 21-nt. In order to displace the stem domain and form a thermodynamically stable structure, each target molecule forms a 24-bp duplex with a designated hairpin strand. The melting temperature of a 24-bp duplex is estimated to be 55 to $65^{\circ} \mathrm{C}$ at $12.5 \mathrm{mM} \mathrm{Mg}^{2+}$ salt concentration and $\mathrm{pH} 8.0^{107}$. The reporter complex consists of a 20-bp duplex with 7-nt toehold. All binding domains are assigned with nucleotides using three letter codes $(A, T, C)$ in order to minimize downstream undesirable leaks and will be discussed in detail in the next paragraph. The next step is to construct the design specification using computer-aid DNA software. A list of available software was mentioned earlier. And for this particular design, NUPACK is a preferable approach.

NUPACK ${ }^{40}$ is open-source software with built-in functions for the design and optimization of DNA sequences based on thermodynamic and kinetic criteria. In brief, the software provides the following functionalities: sequence 
symmetry minimization, finding the average number of incorrect nucleotides relative to target structures at equilibrium, generating the probability map of adopting target structures at equilibrium, and determination of DNA hybridization kinetics. A set of stringent stopping conditions to ensure a higher probability of obtaining desirable target structures can be specified; however, it may take longer to compute and generate such structures. To ensure minimal crosstalk among nine hairpins, multiple design techniques were implemented in NUPACK: i) A 3-letter code was used on the stem region of the hairpin. One of the arms of the stem is "opened" up when an incoming strand displaces it. Note that the other arm always stays double stranded pre- and post- (and during) branch migration process. The arm that stays double stranded was constrained to contain only A, G, and T. This ensures that the arm containing the ' $G$ ' is always in double stranded form. This was so designed because guanine is more promiscuous (binds to both cytosine and thymine), and thus can create undesirable secondary structures when in single stranded state; ii) Clamp domains to minimize the breathing effect at both ends of the duplex; iii) Negative design was used to minimize off-target binding between hairpins during intermediate states and constrained NUPACK to return sequences that maximize 
the probability of a sub-strand (the region of the hairpin that opens the next hairpin) staying single-stranded in intermediate states. The lengths of toehold, stem, and loop domains were optimized using prior reported systems $\mathrm{s}^{63,100,106}$. NUPACK provides the yield of the optimized sequences and defects at equilibrium and in addition, the software avoids improper hybridization of hairpins using Dirks and Pierce's algorithm ${ }^{108}$. The yield of defect is updated after each optimization procedure (changing the sequence composition of stem, loop, toehold, and clamp) and the optimal set of DNA sequences is selected based on the least amount of defect in the yield output. In our system, a list of stopping conditions includes i) target initiator structures cannot form undesirable complexes with more than $5 \%$ of all generated structures and ii) target structures cannot have more than three consecutive guanine bases or more than four consecutive bases of any other nucleotide. The number of trials is set to 10, which means that there are 10 different matching sets of target molecules from the given specification list. The actual sequence composition can be assigned or unassigned. Since we adopted the same reporter complex reported by Zhang et al. ${ }^{61}$, the sequences for the reporter complex are known. As a result, a portion of binding domains in the linear cascade reaction share the same 
sequences as the reporter complex. The script to program target DNA sequences for our system is provided in Appendix A.

\subsection{Chemical Reaction Networks for LCR}

Each DNA sequence is treated as an individual molecule. In the absence of the target initiator molecule, the LCR system remains inactive. Only when the target initiator molecule is present is the LCR activated and the cascade chain reaction initiated, resulting in a linear duplex chain formation. The rate of the linear cascade chain reaction depends on the toehold-binding and branch migration processes. To simulate the formation of various linear duplex lengths, previously reported rate constants were used ${ }^{61,109}$. For instance, the rate constant for displacement of the reporter complex was $1.3 \times 10^{6} \mathrm{M}^{-1} \mathrm{~s}^{-1}$. The rate constant used for toehold-mediated strand displacement was $10^{5} \mathrm{M}^{-1} \mathrm{~s}^{-1}$. Because the toehold binding and branch migration domains between a pair of hairpins have the same length, the strand displacement rate constant is assumed to be equal across all 9 hairpins. Other factors such as the G-C bond having a higher binding energy than the A-T bond, defects in strand synthesis, and non-functional hairpins were not taken into account during simulations. In addition, all hairpin- 
hairpin interactions were assumed to proceed to completion with negligible reverse reaction kinetics.

Each DNA hairpin in the proposed system is treated abstractly as an individual molecule and is assumed to only bind the designated DNA strand strictly via DNA hybridization and strand displacement thermodynamics. The rates of all the reactions in 9 hairpins system can be described based on the second-order kinetic process.

$$
\begin{array}{lll}
I+H 1 \stackrel{k_{0}}{\rightarrow} P 1 & (\mathrm{R} 1) & P 1+H 2 \stackrel{k_{1}}{\rightarrow} P 2 \\
P 2+H 3 \stackrel{k_{2}}{\rightarrow} P 3 & (\mathrm{R} 3) & P 3+H 4 \stackrel{k_{3}}{\rightarrow} P 4 \\
P 4+H 5 \stackrel{k_{4}}{\rightarrow} P 5 & (\mathrm{R} 5) & P 5+H 6 \stackrel{k_{5}}{\rightarrow} P 6 \\
P 6+H 7 \stackrel{k_{6}}{\rightarrow} P 7 & (\mathrm{R} 7) & P 7+H 8 \stackrel{k_{7}}{\rightarrow} P 8 \\
P 8+H 9 \stackrel{k_{8}}{\rightarrow} P 9 & (\mathrm{R} 9) & P 9+R C \stackrel{k_{9}}{\rightarrow} F Q+T E T
\end{array}
$$

Since the number of base pairs from each cascade reaction is equal, we assume the forward rate constants are relatively equivalent for reactions (R1) to (R9). For the rate constant from the reaction (R10), we used the same rate constant reported by Zhang et al. ${ }^{61}$. NUPACK can design DNA sequences and simulate the thermal equilibrium conditions of the given sequence list, but it is 
does not have the capability to analytically solve the above system. To simulate the interactions among hairpins, LBS or DSD can be implemented. It's worth to note that these software treat each DNA strand as a molecule and the time series analysis is done by solving the system of differential equations given by the reaction kinetics that are specified by the users. Given the same principle to perform time-series analysis, we wrote our own solver for the proposed system in Matlab. The Matlab script is provided in Appendix A.

Simulation results of different linear cascade reactions with 9, 6, 4, 2, and 1 hairpin(s) are shown Figure 2.2. As expected, the completion rate of the linear cascade reaction depends on the number of hairpins - less hairpins correspond to a faster completion rate. 


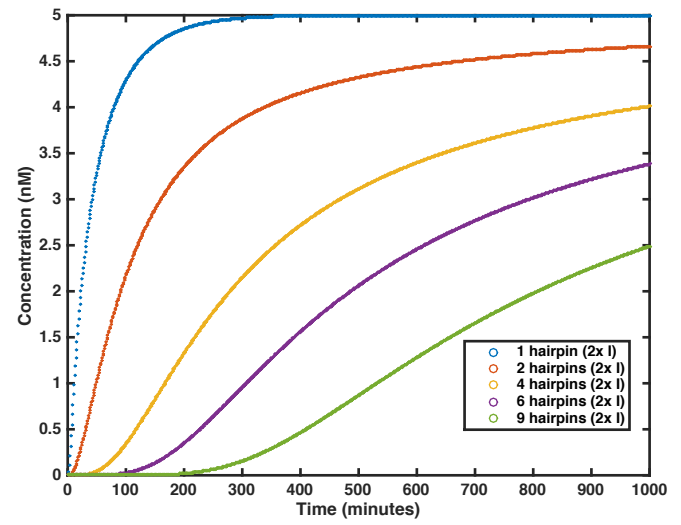

(A)

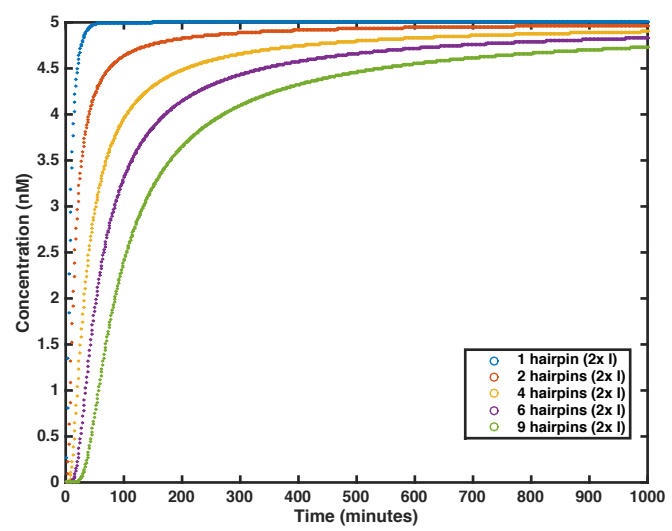

(C)

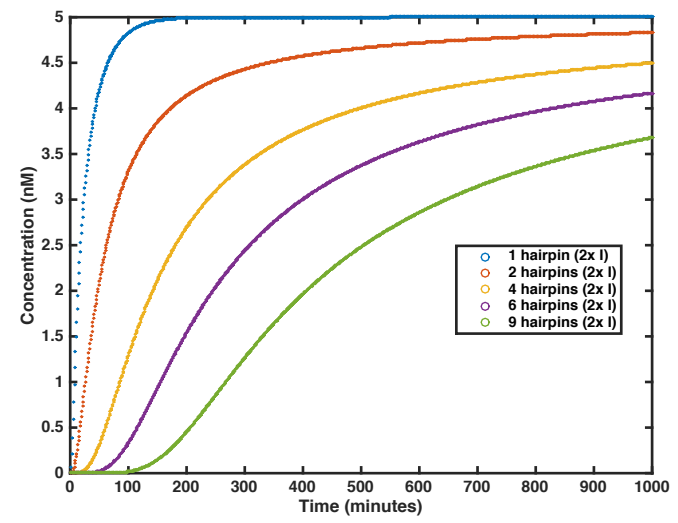

(B)

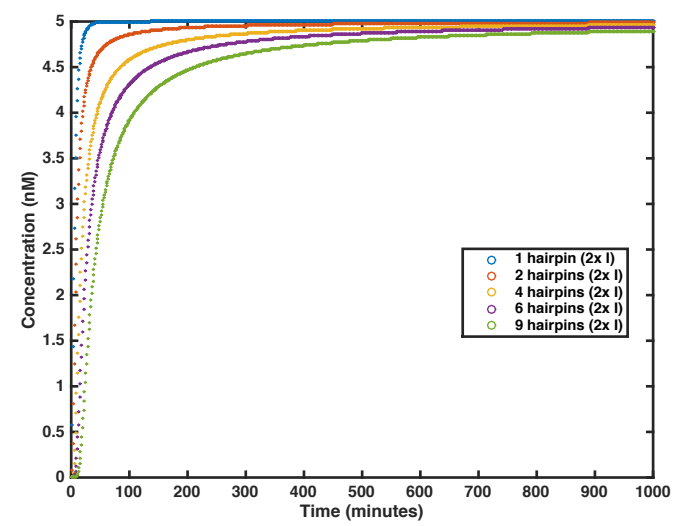

(D)

Figure 2.2: Simulation results of various linear cascade reaction lengths. The length depends on the number of hairpins involved in each reaction. Linear cascade reactions with different rate constants $5 \times 10^{4} \mathrm{M}^{-1} \mathrm{~s}^{-1}(\mathrm{~A}), 10^{5} \mathrm{M}^{-1} \mathrm{~s}^{-1}(\mathrm{~B}), 5 \mathrm{x}$ $10^{5} \mathrm{M}^{-1} \mathrm{~s}^{-1}(\mathrm{C})$, and $1.3 \times 10^{6} \mathrm{M}^{-1} \mathrm{~s}^{-1}(\mathrm{D})$. Simulation is performed based on the assumption that all hairpin-hairpin pairs follow the second order (bimolecular) chemical reaction. Simulation conditions are specified as the following: $5 \mathrm{nM}$ concentration of hairpins, $6.5 \mathrm{nM}$ concentration of reporter complex, $10 \mathrm{nM}$ concentration of initiator, and the rate constant of displacing the reporter complex is common across all simulation experiments.

\subsection{Assembly of LCR System}

All DNA strands were purchased lyophilized from Integrated DNA Technologies and purified using PAGE. In brief, a denaturing PAGE gel was run 
for 90 minutes at $300 \mathrm{~V}$ constant voltage in $1 \mathrm{x}$ TBE buffer. Purified DNA bands were identified, excised, crushed and eluted in elution buffer $(\mathrm{NaCl})$ overnight. The solution containing purified DNA oligonucleotide was centrifuged and the liquid was extracted without disturbing the crushed gel pieces. The solution was washed with $99 \%$ butanol. DNA oligonucleotides were precipitated in pure ethanol and washed with 70\%/30\% ethanol/water. Excess ethanol was removed and the remaining ethanol was removed by vacuum centrifugation. Purified DNA was then re-suspended in 1x TE buffer $\mathrm{pH}$ 8.0. A list of all DNA strands is shown in Table 2.1. Native PAGE analysis was used to identify the effects of the linear cascade DNA hybridization reactions. In brief, each native PAGE was run for 3.5 hours at a constant $170 \mathrm{~V}$. Each well was loaded with $5-10$ picomoles of DNA sample. Ethidium bromide was used to stain DNA for visualizing DNA bands. Ensemble fluorescence spectroscopy was used to monitor the linear cascade chain reaction kinetics of DNA hybridization in solution. A reporter complex consisting of a fluorophore (Tetrachlorofluorescein TET) and a quencher molecule (Iowablack FQ), was adopted from Zhang et. al ${ }^{61}$. Only the last hairpin, when activated, binds to the reporter complex to trigger the fluorescence emission. An equimolar solution of hairpins was prepared. The fluorophore was 
excited at $524 \mathrm{~nm}$ and emission was measured at $541 \mathrm{~nm}$. The excitation and emission slits were set at $10 \mathrm{~nm}$ and $5 \mathrm{~nm}$, respectively. For each experiment 80 $\mu \mathrm{L}$ of the sample (5 $\mathrm{nM}$ of DNA hairpins, $6.5 \mathrm{nM}$ of reporter complex with and without initiator) was combined in quartz cuvettes. In order to prevent DNA loss in the pipetting process (by adhesion to the pipette tips), a non-reactive $20 \mathrm{nt}$ poly-T strand was added to all fluorescence experiments at a concentration of 1 $\mu \mathrm{M}^{61}$. All measurements were performed at $20^{\circ} \mathrm{C}$. Rapid mixing was achieved by carefully but quickly pipetting the whole volume up and down for 10 seconds without generation of air bubbles or loss of material. All experiments were performed in TAE / $12.5 \mathrm{mM} \mathrm{Mg}^{2+}$ buffer solution.

Table 2.1: DNA oligomers for constructing linear cascade DNA hybridization chain reactions. Note: toehold domains (colored green), stem domains (colored blue), clamp domains (colored orange), spacer domains (colored black). In hairpin sequences, stems are underlined. For kinetic studies, RC complex is displaced via the highlighted domain on hairpin $\mathrm{H}_{9}$.

\begin{tabular}{|c|c|c|}
\hline Name & Sequence (5' to 3') & $\mathbf{L}$ \\
\hline Hairpin $\mathrm{H}_{1}$ & $5^{\prime \prime}$ - CCACACATACATCATTCTTACCTATTTTTATT TGATGTATGTGTGGAAGGTT - 3' & 52 \\
\hline Hairpin $\mathrm{H}_{2}$ & 5' - TAGGTAAGAATGATGTATGTGTGGTTTTCTCTTTCATTACATACATCATTCT - 3' & 52 \\
\hline Hairpin $\mathrm{H}_{3}$ & "5' - CATTACATACATCACAACCCACCCTTTTAGAATGATGTATGTAATGAAAGAG - 3' & 52 \\
\hline Hairpin $\mathrm{H}_{4}$ & 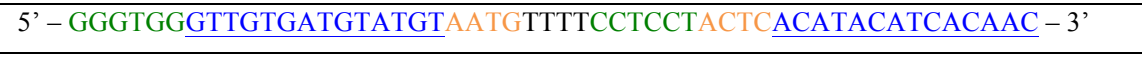 & 52 \\
\hline Hairpin $\mathrm{H}_{5}$ & $5^{\prime \prime}$ - ACTCACATACATCAATCGTCTTTCTTTTGTTGTGATGTATGTGAGTAGGAGG - 3' & 52 \\
\hline Hairpin $\mathrm{H}_{6}$ & 5' - GAAAGACGATTGATGTATGTGAGTTTTTTCTTCCTTTAACATACATCAATCG - 3' & 52 \\
\hline Hairpin $\mathrm{H}_{7}$ & $5^{\prime \prime}$ - TTTAACATACATCACCACTTTCCTTTTTCGAT TGATGTATGTTAAAGGAAGA - 3' & 52 \\
\hline Hairpin $\mathrm{H}_{8}$ & 5' - AGGAAAGTGGTGATGTATGTTAAATTTTCTTCTCCTCCACATACATCACCAC - 3' & 52 \\
\hline Hairpin $\mathrm{H}_{9}$ & 5'-CTCCACATACATCATATTCCCTCATTCAAGCGTGGTGATGTATGTGGAGGAGAAG - 3' & 55 \\
\hline $\mathrm{RC} \mathrm{FQ}$ & 5' - TTGAATGAGGGAATATGATGTATGTGG/Quencher/ - 3' & 27 \\
\hline RC TET & 5' - /Fluorophore/CCACATACATCATATTCCCT - 3' & 20 \\
\hline
\end{tabular}




\begin{tabular}{|c|c|c|}
\hline Initiator $\mathrm{I}_{1}$ & $5^{\prime}$ - AACCTTCCACACATACATCAAATA - 3' & 24 \\
\hline Initiator $\mathrm{I}_{4}$ & 5' - CATTACATACATCACAACCCACCC - 3' & 24 \\
\hline Initiator $\mathrm{I}_{6}$ & $5^{\prime}$ - ACTCACATACATCAATCGTCTTTC - 3' & 24 \\
\hline Initiator $\mathrm{I}_{8}$ & 5' - TTTAACATACATCACCACTTTCCT - 3' & 24 \\
\hline Initiator $\mathrm{I}_{9}$ & 5' - CTTCTCCTCCACATACATCACCAC - 3' & 24 \\
\hline
\end{tabular}

\subsection{Thermal Equilibrium Characterization of LCR}

Analysis of the cascade reaction by polyacrylamide gel electrophoresis (PAGE) is shown in Figure 2.3. In Figure 2.3, lane 1 contains a 20 bp DNA ladder. Lane 2 contains all hairpins but no initiator: products of high molecular weight are almost completely absent, indicating a very low background rate of LCR. Lane 3 contains all hairpins but no initiator (the sample was incubated at room temperature overnight): some evidence of assembly is visible, indicating leak of LCR (leak is defined as undesired reaction in the absence of the initiator strand). Lanes 4 to 8 contain all hairpins with different concentrations of initiator as indicated (samples were incubated at room temperature overnight): strong evidence of assembly is visible, indicating that cascade reactions occurred, as designed. Presence of residual hairpins in those lanes could be due to i) stoichiometric concentration differences between DNA strands and ii) erroneous DNA strands that did not take part in the reaction. By increasing the initiator concentration from $1 x$ to $2 x$ excess (lanes 4 and 5), the products of high molecular 
weight form two distinct bands, indicating that the assembly of 8 hairpins coexists with the assembly of 9 hairpins (evidence in lanes 8 and 9 in Figure 3B). Two distinct high molecular weight products are visible even at higher excess initiator concentration (lanes 6 to 8). In Figure 2.3B, lanes 1 to 9 contain different numbers of hairpins as indicated: products of higher molecular weight are successively visible, indicating that each cascade reaction is achieved as designed. Residual hairpins are visible toward the lower end of the gel. Lane 10 contains a 20 bp DNA ladder. Note that the difference between peaks 8 and 9 hairpin lanes in Figure 2.3B appears smaller than the difference between the two bands in Figure 2.3A. This discrepancy appears to be due to one or both of the following: i) two different comb sizes were used to prepare the gels (i.e. a larger comb size was used in Figure 2.3A than Figure 2.3B), ii) each lane in Figure 2.3A was loaded with 10 pmoles whereas each lane in Figure 2.3B was loaded with 5 pmoles. 


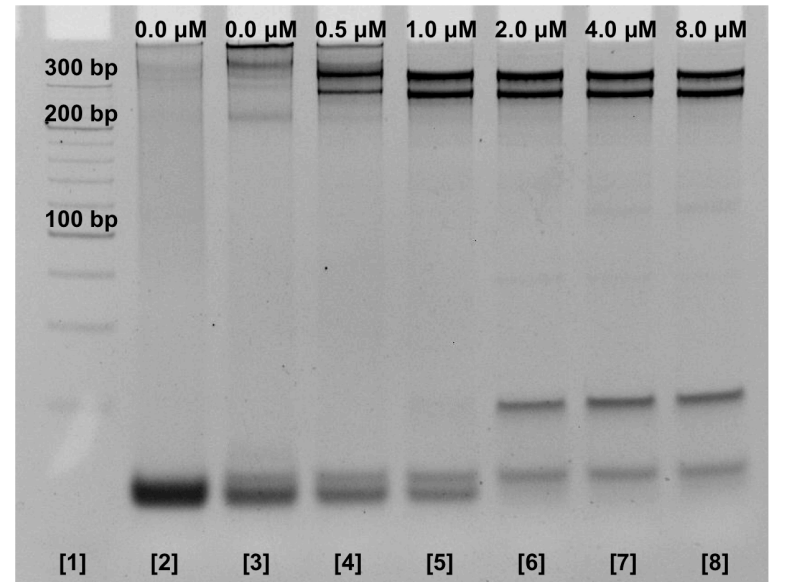

(A)

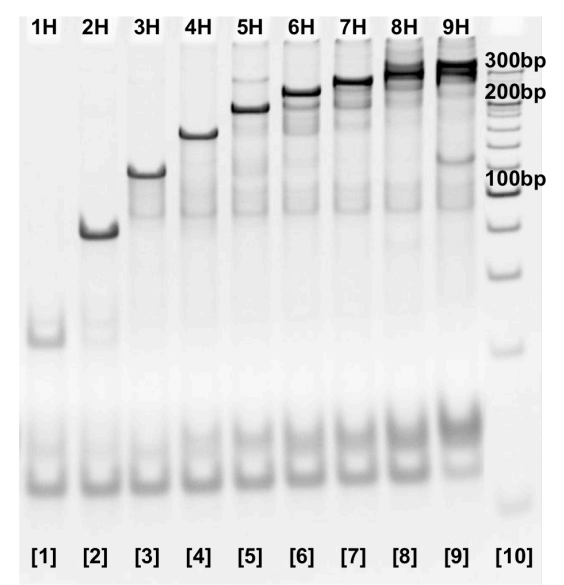

(B)

Figure 2.3: Native PAGE analysis of LCR: All samples were incubated at room temperature for at least 15 hours. (A) Effect of initiator concentration on LCR. All samples were prepared at $500 \mathrm{nM}$ and $20 \mu \mathrm{L}$ of sample was loaded into each lane. Lane 1: DNA marker with 20-bp increments $(20-300 \mathrm{bp})$; Lane 2: $0 \mu \mathrm{M}$ of initiator and $0.5 \mu \mathrm{M}$ mixture of $\mathrm{H} 1$ to $\mathrm{H} 9$ without overnight incubation. Lanes 3 to 8: six different concentrations of initiator $(0,0.5,1,2,4,8 \mu \mathrm{M})$ in a $0.5 \mu \mathrm{M}$ mixture of $\mathrm{H}_{1}$ to $\mathrm{H}_{9}$. (B) Step-by-step of the assembly of cascade chain reaction. The initiator was added 1x. All samples were prepared at $100 \mathrm{nM}$ and $50 \mu \mathrm{L}$ of sample was loaded into each lane. Subsequent higher gel bands correspond to longer linear duplexes being formed. The largest linear duplex is resulted from the assembly of linear cascade DNA hybridization of 9 distinct hairpins. Lane 1: $\mathrm{H}_{1}+$ target DNA initiator; Lane 2: $\mathrm{H}_{1}+\mathrm{H}_{2}+$ target DNA initiator; Lane 3: $\mathrm{H}_{1}+\mathrm{H}_{2}$ $+\mathrm{H}_{3}+$ target DNA initiator; Lane $4: \mathrm{H}_{1}+\mathrm{H}_{2}+\mathrm{H}_{3}+\mathrm{H}_{4}+$ target DNA initiator; Lane 5: $\mathrm{H}_{1}+\mathrm{H}_{2}+\mathrm{H}_{3}+\mathrm{H}_{4}+\mathrm{H}_{5}+$ target DNA initiator; Lane 6: $\mathrm{H}_{1}+\mathrm{H}_{2}+\mathrm{H}_{3}+\mathrm{H}_{4}+\mathrm{H}_{5}+$ $\mathrm{H}_{6}+$ target DNA initiator; Lane 7: $\mathrm{H}_{1}+\mathrm{H}_{2}+\mathrm{H}_{3}+\mathrm{H}_{4}+\mathrm{H}_{5}+\mathrm{H}_{6}+\mathrm{H}_{7}+$ target DNA initiator; Lane 8: $\mathrm{H}_{1}+\mathrm{H}_{2}+\mathrm{H}_{3}+\mathrm{H}_{4}+\mathrm{H}_{5}+\mathrm{H}_{6}+\mathrm{H}_{7}+\mathrm{H}_{8}+$ target DNA initiator; Lane 9: $\mathrm{H}_{1}+\mathrm{H}_{2}+\mathrm{H}_{3}+\mathrm{H}_{4}+\mathrm{H}_{5}+\mathrm{H}_{6}+\mathrm{H}_{7}+\mathrm{H}_{8}+\mathrm{H}_{9}+$ target DNA initiator; Lane 10: DNA marker with 20-bp increments $(20-300 \mathrm{bp})$.

Control analysis of the linear cascade reaction with different number of hairpins is shown in Figure 2.4. In gel A, only hairpins 8 and 9 are characterized 
with and without the initiator ( $(\mathrm{I} 8)$. Lane 2 contains a mixture of hairpins 8 and 9: products of high molecular weight are almost absent. Lane 3 contains a mixture of hairpins 8 and 9 subjected to a quick $90^{\circ} \mathrm{C}$ anneal for 10 minutes: products of high molecular weight are visible but with little evidence that the cascade reaction occurred. Lane 4 contains hairpin 8 with the initiator: products of high molecule weight are visible, indicating that the hairpin and initiator formed as anticipated. Lane 5 contains both hairpins 8 and 9 with the initiator: products of high molecular weight are visible, indicating that the linear cascade reaction occurred. Residual hairpins in lanes $4-5$ indicate a mismatch between hairpin concentrations. In gel B, only hairpins $6,7,8$, and 9 are characterized with and without the initiator $\left(\mathrm{I}_{6}\right)$. Lanes 2, 3, and 4 contain a mixture of two hairpins $\left(\mathrm{H}_{6-}\right.$ 7), three hairpins $\left(\mathrm{H}_{6-8}\right)$, and four hairpins $\left(\mathrm{H}_{6-9}\right)$, respectively without the initiator: products of high molecular weight are slightly visible, indicating evidence of some leak. Lanes 5, 6, 7 contain a mixture of $\mathrm{H}_{6-7}, \mathrm{H}_{6-8}$, and $\mathrm{H}_{6-9}$, respectively with the initiator: products of high molecular weight are visible, indicating strong evidence of linear cascade reactions. A mismatch of hairpin concentrations is also visible in lines 7 and 8 . In gel C, only hairpins 4, 5, 6, 7, 8, and 9 are characterized with and without the initiator $\left(\mathrm{I}_{4}\right)$. Lanes $2-6$ contain a mixture of two hairpins 
$\left(\mathrm{H}_{4-5}\right)$, three hairpins $\left(\mathrm{H}_{4-6}\right)$, four hairpins $\left(\mathrm{H}_{4-7}\right)$, five hairpins $\left(\mathrm{H}_{4-8}\right)$, and six hairpins $\left(\mathrm{H}_{4-9}\right)$ without the initiator: most of hairpins stay as monomers and little evidence of cascade reaction is visible. Lanes 7 - 11 contain a mixture of $\mathrm{H}_{4-5}, \mathrm{H}_{4-6}$, $\mathrm{H}_{4-7}, \mathrm{H}_{4-8}$, and $\mathrm{H}_{4-9}$ with the initiator: products of high molecular weight are visible, indicating that strong linear cascade reactions are initiated by the initiator. Lanes 5, 8, and 11 in gels A, B, and C show high molecular weight of linear oligomer products formed, indicating strong evidence of linear cascade DNA hybridization chain reactions as anticipated. 


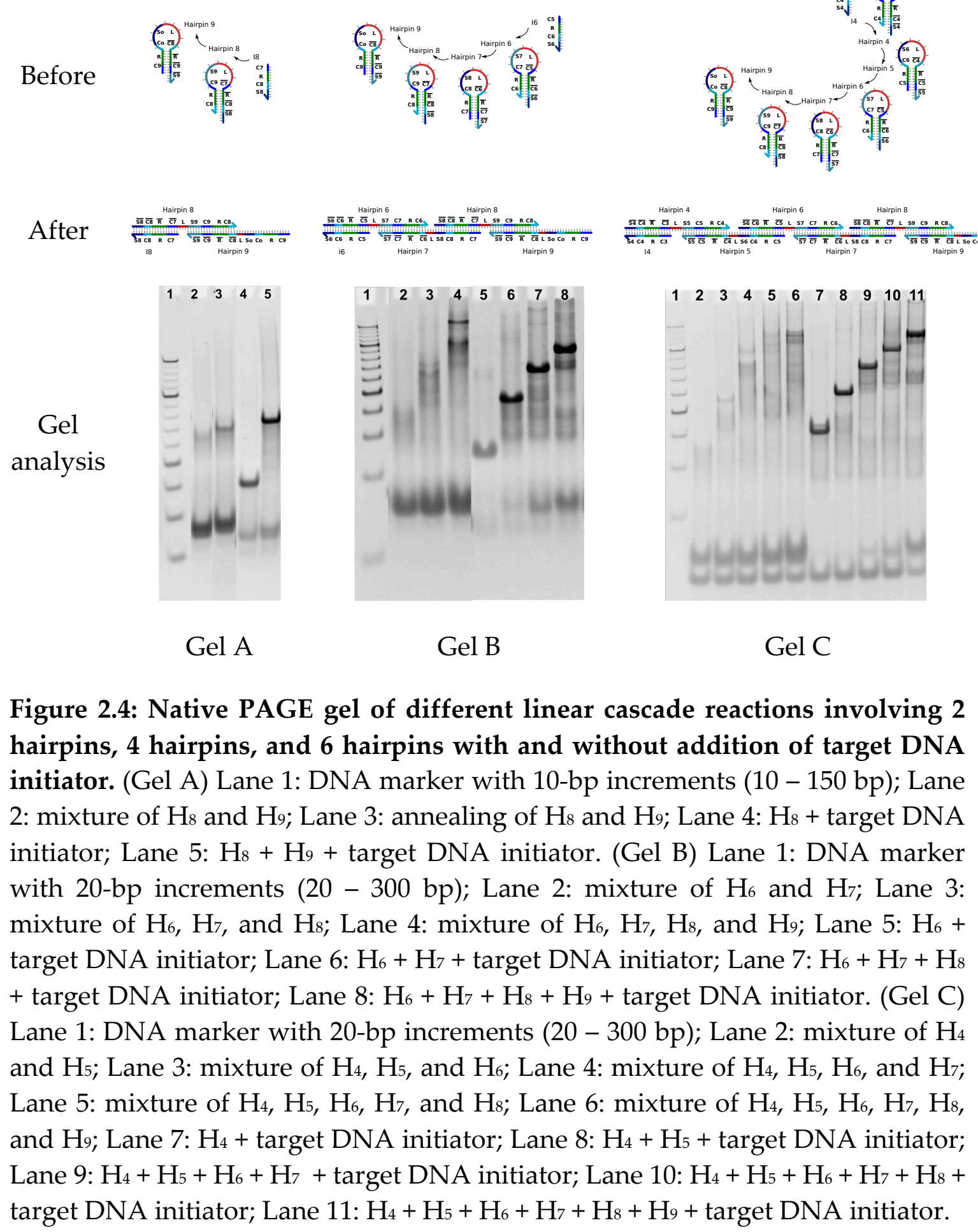




\subsection{Kinetic Characterization of LCR}

The kinetics of linear cascade DNA hybridization chain reactions (LCR) can be explored using fluorescence emissions. The reporter complex is initially quenched due to the close proximity of the fluorophore and quencher, which are constrained by duplex formation (Figure 2.5A). Upon hybridization, the reporter complex is displaced by the open arm of hairpin 9, resulting in an increase in fluorescence emission. Figure 2.5B shows real-time kinetic characterization of the linear cascade DNA hybridization chain reaction of nine distinct hairpins. All fluorescence emissions were normalized to the concentration of the output of the last hairpin $\left(\mathrm{H}_{9}\right)$; the normalization technique was adopted from prior studies ${ }^{61}$. In this case, each opened hairpin 9 is assumed to bind to the reporter complex. In Figure 2.5B, no fluorescence emission is observed while monitoring the kinetic of LCR in the absence of initiator (black curve) for more than 10 hours, indicating that the linear cascade chain reaction does not occur in the absence of the initiator (I). Addition of $\mathrm{I}$ to the hairpin mixture at $1 \mathrm{x}$ concentration leads to rapid fluorescence emission for up to 200 minutes which slowly increases as equilibrium is reached, provide strong evidence of the linear cascade chain reaction (red curve). In the presence of excess initiator, the LCR system yields

minimal difference in fluorescence signal compared to $1 \mathrm{x}$ concentration of 46 
initiator (green curve), demonstrating that the linear cascade chain reaction is sufficient at $1 \mathrm{x}$ concentration, although equilibrium gel analysis (Figure 2.3A lane 5) indicates minimal leftover hairpins in the presence of $2 x$ initiator concentration. Figure 2.5B shows the effect of a leak (an undesired reaction) in the absence of the initiator as a function of hairpin concentrations - a higher concentration causes more leak. This result confirms the challenge of leak when constructing DNA systems to operate at high concentrations. Even though DNA strands were purified using a denaturing PAGE method, leaks are difficult to eliminate. At $5 \mathrm{nM}$ concentration, our LCR system leak occurs at a slower rate than the actual reaction as shown in Figure 2.5A. Alternative approaches to conquer leak include: i) tethering DNA systems on a surface of DNA nanostructures ${ }^{104,105,110-113}$, or ii) incorporating double or triple length domain motifs $^{38}$. Note that the fluorescence data for leaks at $50 \mathrm{nM}$ indicates significant leaks as compared to the gel data for leaks at $500 \mathrm{nM}$ of the same system at equilibrium in Figure 2.3A lane 3. This discrepancy appears to be due to any of the following: i) the fluorescence method relied on the signal readout from the reporter complex whereas the gel method relied on the nanostructure formation directly due to just hairpins, ii) improper synthesis of the last hairpin, which 
could cause interaction directly with the reporter complex, and thus produce a false positive fluorescence signal in addition to the actual fluorescence leak resulting from triggering LCR in the absence of the initiator strand, or iii) the gel images in Figure 2.3B and Figure 2.4 do show a detectable amount of leak, while the gel image in Figure 2.3A does not show many intermediate bands as part of the leak, just DNA bands at the top of the lane. It is possible that at higher concentration, the intermediate products shifted/aggregated to form larger products that can be seen as bands at the top of lane 3 . 

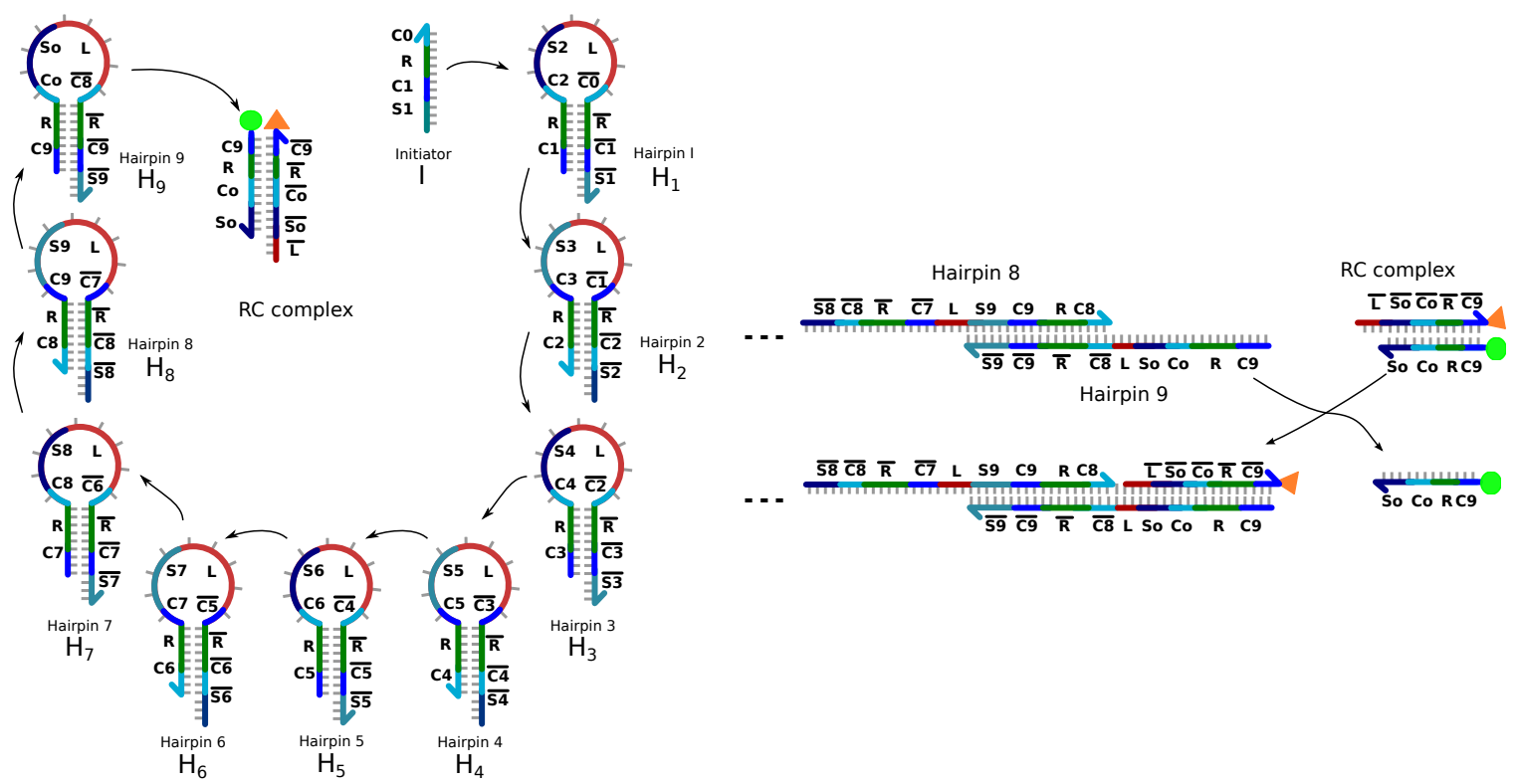

(A)

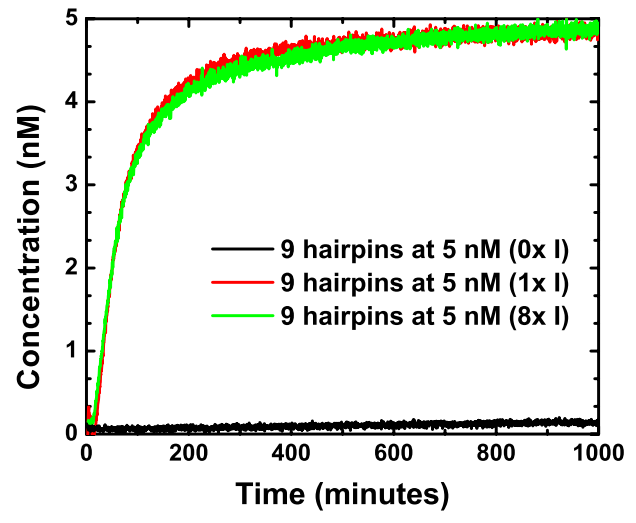

(B)

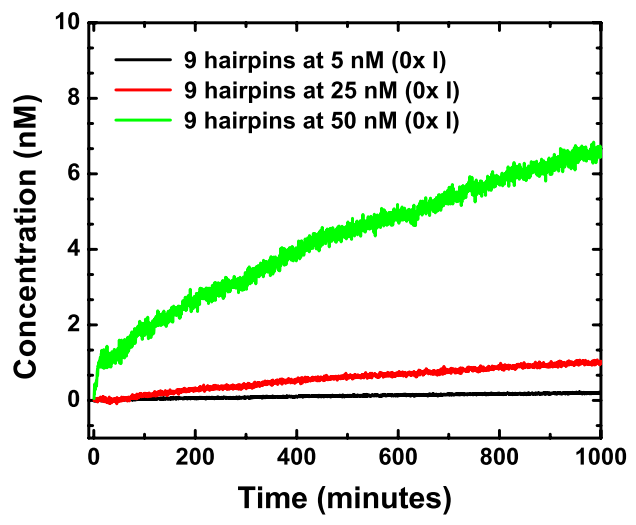

(C)

Figure 2.5: Kinetic characterization of the linear cascade DNA hybridization chain reactions. All samples were prepared at $5 \mathrm{nM}$ of hairpin with $6.5 \mathrm{nM}$ of reporter complex. (A) Schematic of LCR system before and after interacting with the reporter complex. (B) Effect of initiator concentration on the rate of linear cascade reaction. Hairpins do not hybridize in the absence of initiator (9 hairpins $+0 \mathrm{x}$ I, black). The linear cascade reaction occurs in the presence of $1 \mathrm{x}$ initiator concentration (9 hairpins $+1 x$ I, red). The linear cascade reaction occurs at the same rate in the presence of excess initiator (9 hairpins $+8 x \mathrm{I}$, green). (C) Effect of hairpin concentration on the rate of crosstalk and leak reactions: $5 \mathrm{nM}$ (black 
curve), $25 \mathrm{nM}$ (red curve), and $50 \mathrm{nM}$ (green curve) without the presence of initiator.

The effect of the number of hairpins on the rate of the linear cascade reaction is shown in Figure 2.6A. The dotted horizontal line indicates the half-life of the linear cascade reaction (i.e. the time required for the reaction to reach $50 \%$ completion). All experimental data was collected at $5 \mathrm{nM}$ concentration. Initiating the linear cascade chain reaction with a single hairpin results in a rapid fluorescence emission which slowly increases as equilibrium is reached (black curve). The linear cascade chain reaction reaches 50\% completion within 1.47 minutes. The linear cascade chain reaction involving nine hairpins (cyan curve) takes approximately 41.97 minutes to reach $50 \%$ completion, roughly 28 times slower than the reaction involving a single hairpin. To reach $50 \%$ completion, the linear cascade reactions involving two (red curve), four (green curve), and six (blue curve) hairpins were measured to be $8.84,18.78$, and 27.98 minutes respectively. As expected from simulation and experimental results, a lower number of hairpins results in a faster linear cascade reaction and vice versa. Experimental data indicates that the half-time completion of our LCR system follows a linear behavior (Figure 2.6B). As more hairpins are involved in the 
linear cascade reaction, more time is required to reach the half-time completion. The linear behavior provides an additional criterion for the design and scaling of new DNA circuits.

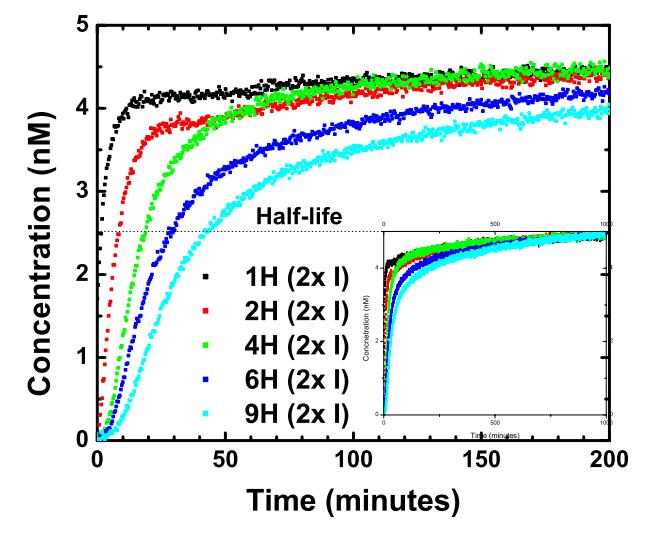

(A)

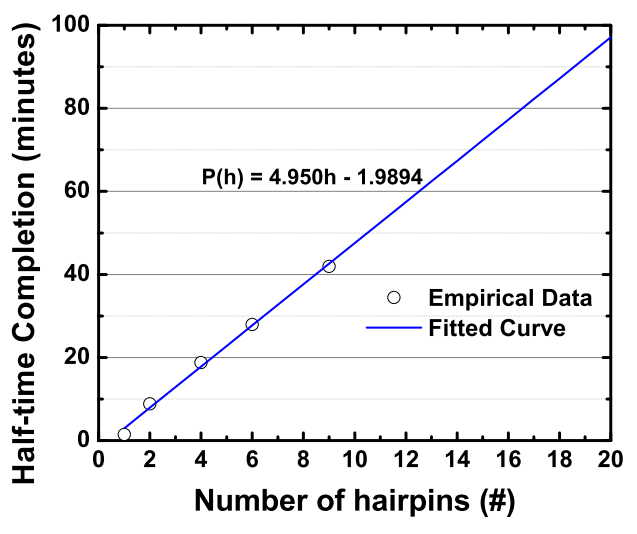

(B)

Figure 2.6: (A) Effect of the number of hairpins on the rate of linear cascade reaction: 1 hairpin (black curve), 2 hairpins (red curve), 4 hairpins (green curve), 6 hairpins (blue curve), 9 hairpins (cyan curve). Note: the dotted horizontal line indicates the half-life of the linear cascade reaction (the time required for the reaction to reach $50 \%$ completion). Inset shows the real-time kinetic of all linear cascade reactions at equilibrium. (B) Empirical projection of the half-time completion as a function of hairpins. Note: each circle is an experimental result corresponding to the number of hairpins participating in the linear cascade reaction; a blue line is a fitted curve from those circles.

\subsection{Model Validation and Synthesis Yield}

The experimental results from Figure 2.6A were fitted using the least squares method ${ }^{61}$. Each experimental result was fitted to the corresponding cascade simulation. For example, the experimental result of nine hairpins was fitted using the cascade simulation of nine hairpins. As mentioned from prior 
discussion, the hairpins were designed to have the same structure with different nucleotide composition. The model assumed that the rate constant of each cascade reaction was equal. To determine this rate constant, the least squares method was run until a final best-fit curve was obtained. This best-fit curve was resulted from minimizing the error function, which was the least squares error between the experimental data and the simulation data. Figure 2.7 shows the results from fitting the experimental data to the corresponding cascade simulations. The rate constants for systems of 1,2, 4, 6, and 9 hairpins were fitted to be $0.974 \times 10^{6} \mathrm{M}^{-1} \mathrm{~s}^{-1}, 0.815 \times 10^{6} \mathrm{M}^{-1} \mathrm{~s}^{-1}, 0.582 \times 10^{6} \mathrm{M}^{-1} \mathrm{~s}^{-1}, 1.03 \times 10^{6} \mathrm{M}^{-1} \mathrm{~s}^{-1}$, and $1.22 \times 10^{6} \mathrm{M}^{-1} \mathrm{~s}^{-1}$, respectively. These rate constants are independent of the cascade length and are approximately similar to the rate constant of the reporter complex $\left(1.3 \times 10^{6} \mathrm{M}^{-1} \mathrm{~s}^{-1}\right)$. Note that the reporter complex was adopted from Zhang et al. ${ }^{61}$ Note that the fitted data reached the saturation level earlier than the experimental data. This discrepancy may be due to one or both of the following: i) improper synthesis or defects of DNA, and ii) the model does account for the fact that the actual system is not $100 \%$ efficient (i.e. all molecules from the model are assumed to participate in the reaction and this notion seems to be far-off from the actual observed experimental data). 


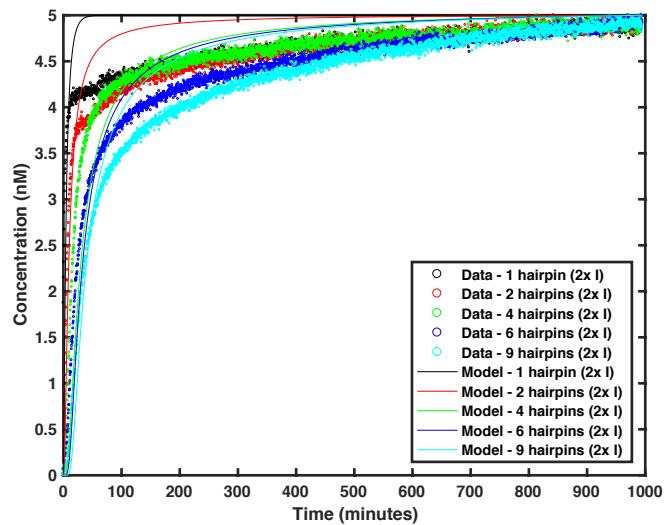

Figure 2.7: Fitting the experimental results using the proposed model. Scatter plots are experimental data; Line curves are fitted data using the least squares method.

Although linear behavior has been observed in our systems, the yield of the cascade reaction is difficult to qualitatively obtain from our gel data or fluorescence data. One possible approach to roughly estimate the yield of the cascade reaction is to compare the raw fluorescence data among various linear cascade reaction chains (Figure 2.8). Ideally, the maximum fluorescence intensity at thermal equilibrium of different numbers of hairpins participating in the linear cascade reaction would reach the same level. However, the actual fluorescence data indicate that the maximum fluorescence intensity at thermal equilibrium of a linear cascade reaction consisting of a single hairpin is higher than that of a linear cascade reaction consisting of nine hairpins. Assuming that i) the maximum fluorescence intensity at thermal equilibrium is correlated with the 
yield of hairpins participating in the linear cascade reaction and ii) the yield of a last hairpin participating in the linear cascade reaction is $100 \%$, the yield of the cascade reaction consisting of 9 hairpins is approximately 79\% (Figure 2.8). 


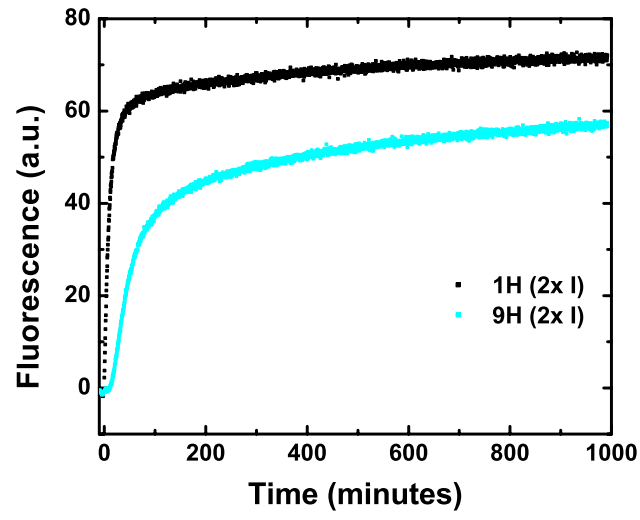

(A)

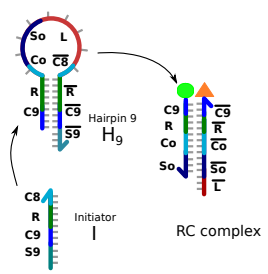

Schematic B

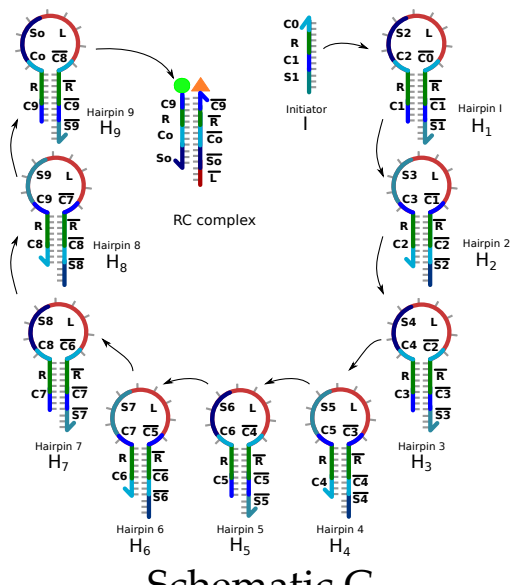

Schematic C

Figure 2.8: Estimation of yield via maximum fluorescence intensity readout. All experimental data were collected at $5 \mathrm{nM}$ concentration. Assume that the maximum fluorescence intensity at thermal equilibrium is correlated with the yield of hairpins participating in the linear cascade reaction. Black curve is the fluorescence emission of initiating the last hairpin and resulting in displacing the reporter complex (schematic B). Cyan curve is the fluorescence emission of initiating the first hairpin and resulting in displacing the reporter complex after the linear cascade reaction (schematic $\mathrm{C}$ ). The maximum fluorescence at thermal equilibrium of the linear cascade consisting of a single hairpin is 72 a.u. The maximum fluorescence at thermal equilibrium of the linear cascade consisting of 9 distinct hairpins is 57 a.u. To the first order approximation, the yield of 9 hairpins is approximated $79 \%$.

An alternative approach to estimate the yield is to employ a quantitative calibrated DNA ladder via gel electrophoresis or to directly measure the final linear structures via scanning probe microscopy. To avoid non-completion 
reactions, stoichiometry among DNA strands must be preserved and erroneous DNA strands must not participate in the reaction. In addition to these proposed approaches, different versions of hairpins can be tuned to determine the causes of additional peaks in the gel data.

\subsection{Conclusion}

We have demonstrated the design, simulation, and synthesis of linear cascade DNA hybridization chain reactions by non-equilibrium assembly. Cascade reactions provide a potential strategy to reduce short conventional stepby-step reactions in a highly efficient and elegant fashion. Cascade reactions have been shown to work in many dynamic DNA systems. We have developed a linear cascade DNA hybridization chain reaction consisting of nine distinct DNA hairpins. Gel analysis and reaction kinetics indicate strong evidence of the designed assembly. For scaling up DNA circuits, empirical data show that halftime completion follows linear behavior as the number of hairpins increases. This method can be used for constructing more complex linear cascade reactions on the surface of DNA nanostructures as well as for realizing nano-breadboard DNA circuits. 


\section{Localized DNA Hybridization Chain Reactions on DNA Nanostructures}

Given that the sequence design and simulation can be accomplished within reasonable basis, the next step is to integrate DNA hybridization reactions on 2D surfaces. Chapter 3 demonstrates a novel construction of localized DNA hybridization chain reactions on small DNA nanostructures. This chapter was published as: Hieu Bui, Vincent Miao, Sudhanshu Garg, Reem Mokhtar, Tianqi Song and John Reif, "Design and analysis of localized DNA hybridization chain reactions", Small 1602983 (2017). DOI: http://dx.doi.org/10.1002/smll.201602983

\subsection{Background}

Theoretical models of localized DNA hybridization reactions on nanoscale substrates indicate potential benefits over conventional DNA hybridization reactions. Recently, a few approaches have been proposed to speed up DNA hybridization reactions; however, experimental confirmation and quantification of the acceleration factor have been lacking. Here we present a system to investigate localized DNA hybridization reactions on a nanoscale substrate. Our system consists of six metastable DNA hairpins that are tethered to a long DNA

track. The localized DNA hybridization reaction of the proposed system is triggered by a DNA strand which initiates the subsequent self-assembly. 
Fluorescence kinetics indicates that the half-time completion of a localized DNA hybridization chain reaction is six times faster than the same reaction in the absence of the substrate. The proposed system provides one of the first known quantifications of the speed-up of DNA hybridization reactions due to the locality effect.

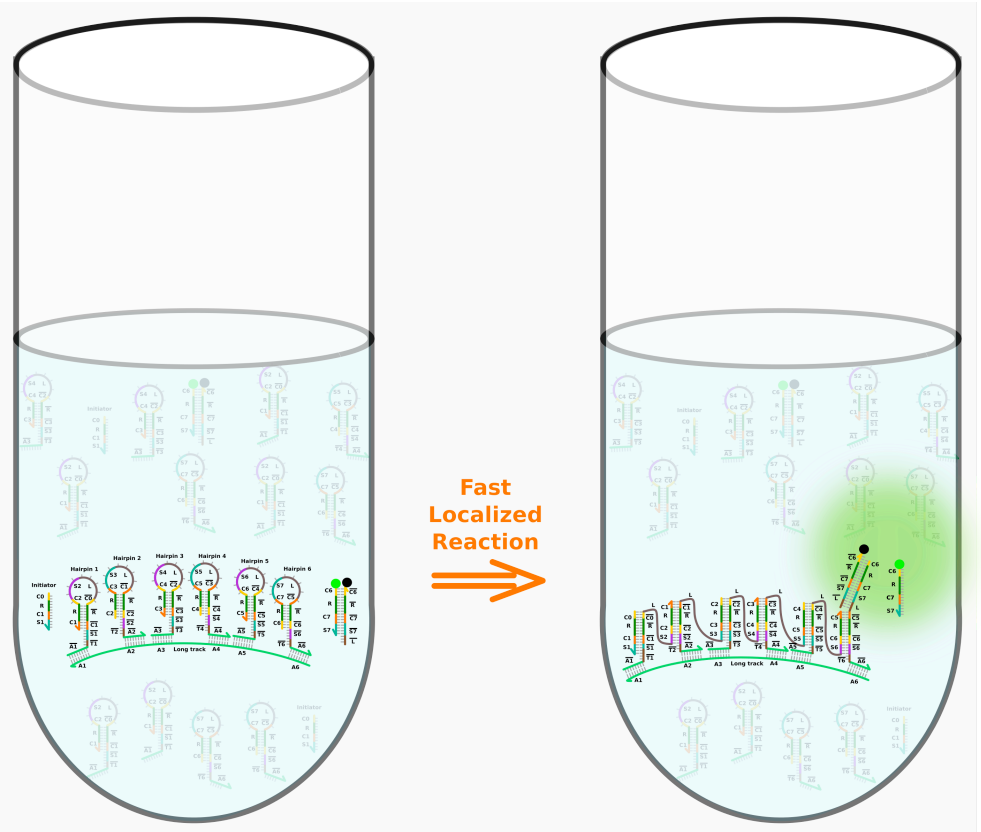

Figure 3.1: Depiction of localized DNA hybridization chain reactions on DNA nanotrack. The presence of the surface enables the reaction to proceed faster.

\subsection{Localized DNA Hybridization Reactions}

DNA hybridization reactions based on toehold-mediated strand displacement have been studied in the past decade and widely used to operate nanoscale machines and construct DNA circuits. ${ }^{4,26,27,30,32,33,59,61,97,114-117}$ The kinetics 
of DNA hybridization reactions depend on diffusion of DNA molecules which randomly collide and interact in a three-dimensional fluidic space. To speed up DNA hybridization reactions, a high concentration of DNA molecules is often employed; however, leaks and crosstalk frequently occur in the reactions at high concentrations. As a result, unintended DNA hybridization reactions impede the desired DNA hybridization reactions from happening. Recently, theoretical models to describe DNA hybridization reactions on nanoscale substrates have been proposed. 5,104,113,118 The models indicate strong evidence of speed-up as well as leak reduction when DNA gates are tethered to the substrates. In terms of experimental demonstration, Kopperger et al. studied the diffusive transport mechanism of linear DNA strands bound to the surface of DNA origami rectangle using surface-bound sticky strands. ${ }^{112}$ Teichmann et al. expanded the work of Kopperger et al. by exploring the effect of spatial arrangement of DNA strands on the surface of DNA origami. ${ }^{119}$ From understanding the spatial arrangement of DNA molecules on nanoscale surfaces, Ruiz et al. created a simple see-saw DNA amplifier circuit that could operate on the surface of DNA origami. ${ }^{120}$ Prior studies often tethered DNA strands to the surface of DNA nanostructures to exploit the locality effect. However, the strands are free to 
diffuse beyond the reaction volume during hybridization. When the DNA strands are not physically attached to the surface, the likelihood of having DNA strands flow away from the surface during hybridization is higher than when the strands are physically tied down to the surface. Dunn et al. studied the mechanism and kinetics of strand displacement of 16-bp DNA duplex with 16-nt sticky end on quartz crystal microbalance as a function of sequence length, concentration, and G/C content ${ }^{121}$. Recently Yin et al. employed triggered selfassembly hybridization chain reaction $(\mathrm{HCR})^{100-102,122,123}$ to program DNA that could form and grow nanoscale structures ranging from small oligomers to branched oligomers and even dendritic oligomers. ${ }^{63,124}$ Inspired by the triggered self-assembly HCR and the potential speed-up from tethering DNA reactions to the surface, we present a system to study DNA hybridization chain reactions on a nanoscale surface. In particular, the proposed system consists of six metastable DNA hairpins that are bound to a long 90-nucleotide DNA strand. The use of metastable DNA hairpin provides the following advantages over the regular DNA strand: i) it acts as an anchor to fasten the entire structure to substrates such as DNA nanostructures via DNA hybridization or other surfaces via chemistry linkers like biotin-streptavidin, ii) it acts as a carrier to transport cargo 
from one position to the other, and iii) it acts as a flexible linker to connect the carrier to the anchor, thus preventing the cargo from flowing away during hybridization. Experimental results provide strong evidence that the speed-up of DNA hybridization reactions which is due to the locality effect.

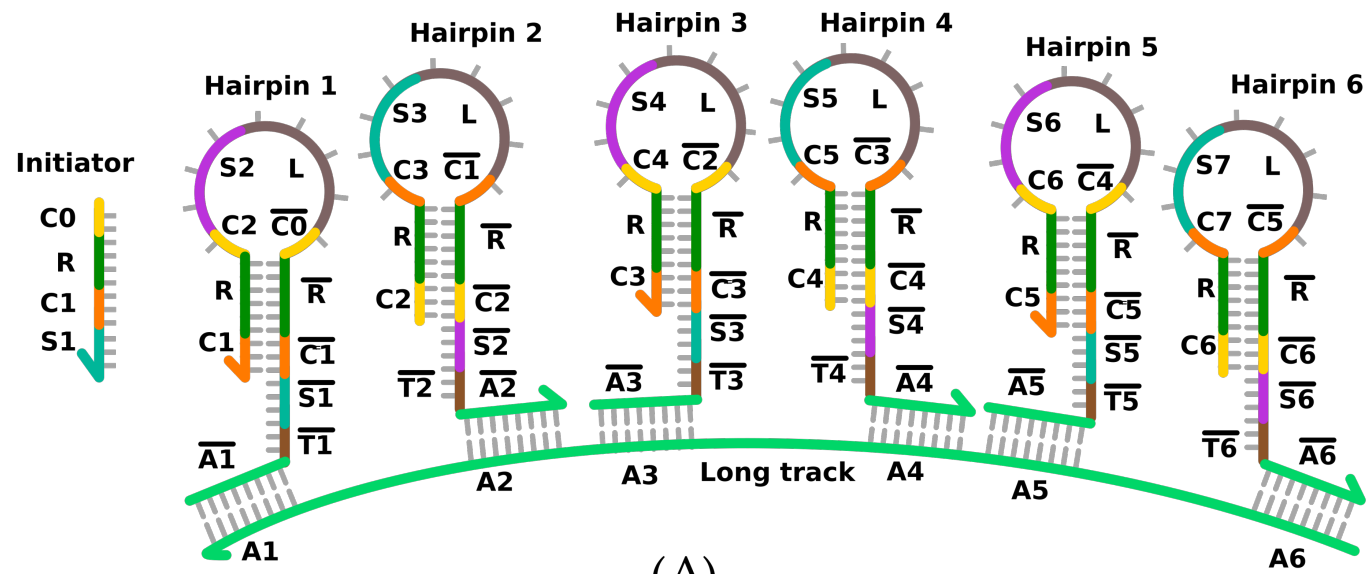

(A)
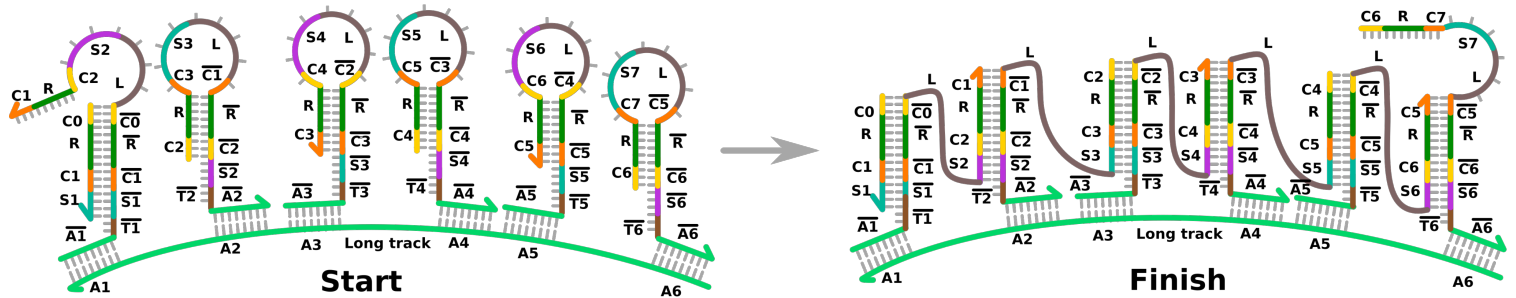

(B)
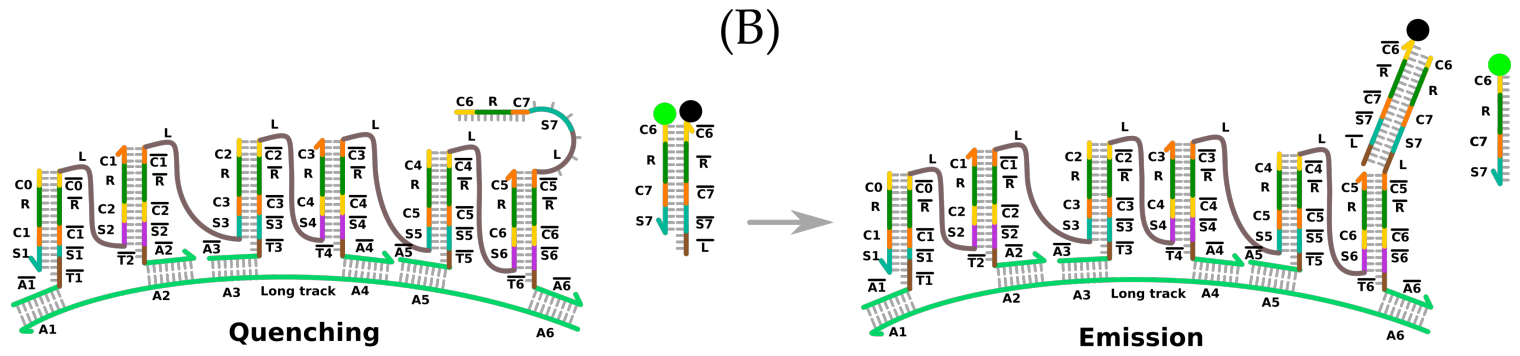

(C)

Figure 3.2: (A) Components of localized DNA hybridization chain reaction system: six metastable DNA hairpins anchored to a long DNA track. Prior to the addition of the initiator, DNA hairpins do not hybridize. (B) Triggered selfassembly of localized DNA hybridization chain reaction mechanism for the first 
and last steps. (C) Fluorescence reporting mechanism to observe the kinetics of localized DNA hybridization reaction.

\subsection{Sequence Design}

A specific design of localized DNA hybridization chain reactions consists of a long DNA strand (LT), a set of six metastable DNA hairpins $\left(\mathrm{H}_{1}, \mathrm{H}_{2}, \mathrm{H}_{3}, \mathrm{H}_{4}\right.$, $\mathrm{H}_{5}, \mathrm{H}_{6}$ ), and an initiator (I) as shown in Figure 3.2. The long DNA strand, also called the DNA track, provides complementary binding domains to assemble the six hairpins together. Each DNA hairpin consists of a stem, a loop, and a sticky end. The stem consists of two domains ( $C_{i}$ and $\left.R\right)$ to form a stable duplex with 14 base pairs. The loop consists of two clamp domains ( $\mathrm{C}_{\mathrm{i}-1}$ and $\left.\mathrm{C}_{\mathrm{i}+1}\right)$, a linker domain $(\mathrm{L})$, and a sequestered domain $\left(\mathrm{S}_{\mathrm{i}+1}\right)$. The two clamp domains within the loop are programmed to both ensure the incoming hairpin from going back to the previous state (i.e. $\mathrm{C}_{\mathrm{i}-1}$ ) and to provide a place to fasten the next hairpin (i.e. $\mathrm{C}_{i+1}$ ) upon hybridization. The linker domain is served as a flexible rope and can be tuned to accommodate for different spatial arrangements among hairpins. Prior systems ${ }^{100,122}$ had to be tailored to have a stem of 18 base pairs and a toehold/hairpin loop region of $6 \mathrm{nt}$. The clamp domains enable us to design systems with variable stem and toehold lengths, thus it increases the sequence space and applicability. The sticky end consists of an external toehold domain 
$\left(\mathrm{S}_{\mathrm{i}}\right)$ that is complementary to the loop domain $\left(\mathrm{S}_{\mathrm{i}+1}\right)$ of hairpin $\left(\mathrm{H}_{\mathrm{i}+1}\right)$, a spacer domain $\left(\mathrm{T}_{\mathrm{i}}\right)$ to offset potential geometrical constraints, and an anchor domain $\left(\mathrm{A}_{\mathrm{i}}\right)$ to attach the hairpin $\left(\mathrm{H}_{\mathrm{i}}\right)$ to the long DNA track. Hairpins are distinguishable by two single-stranded toehold domains $\left(S_{i}\right.$ and $\left.S_{i+1}\right)$ - one toehold is external (a single-stranded sticky end, readily available for hybridization) and one is sequestered within the hairpin loop. The stem (R) and linker (L) domains of all hairpins comprise of the same sequences. The spacer domain (T) is used to accommodate for potential geometrical constraints. The clamp domain (C) is implemented to minimize the breathing effect at the ends of the DNA duplex and to ensure that the reaction proceeds forward. ${ }^{106}$ 

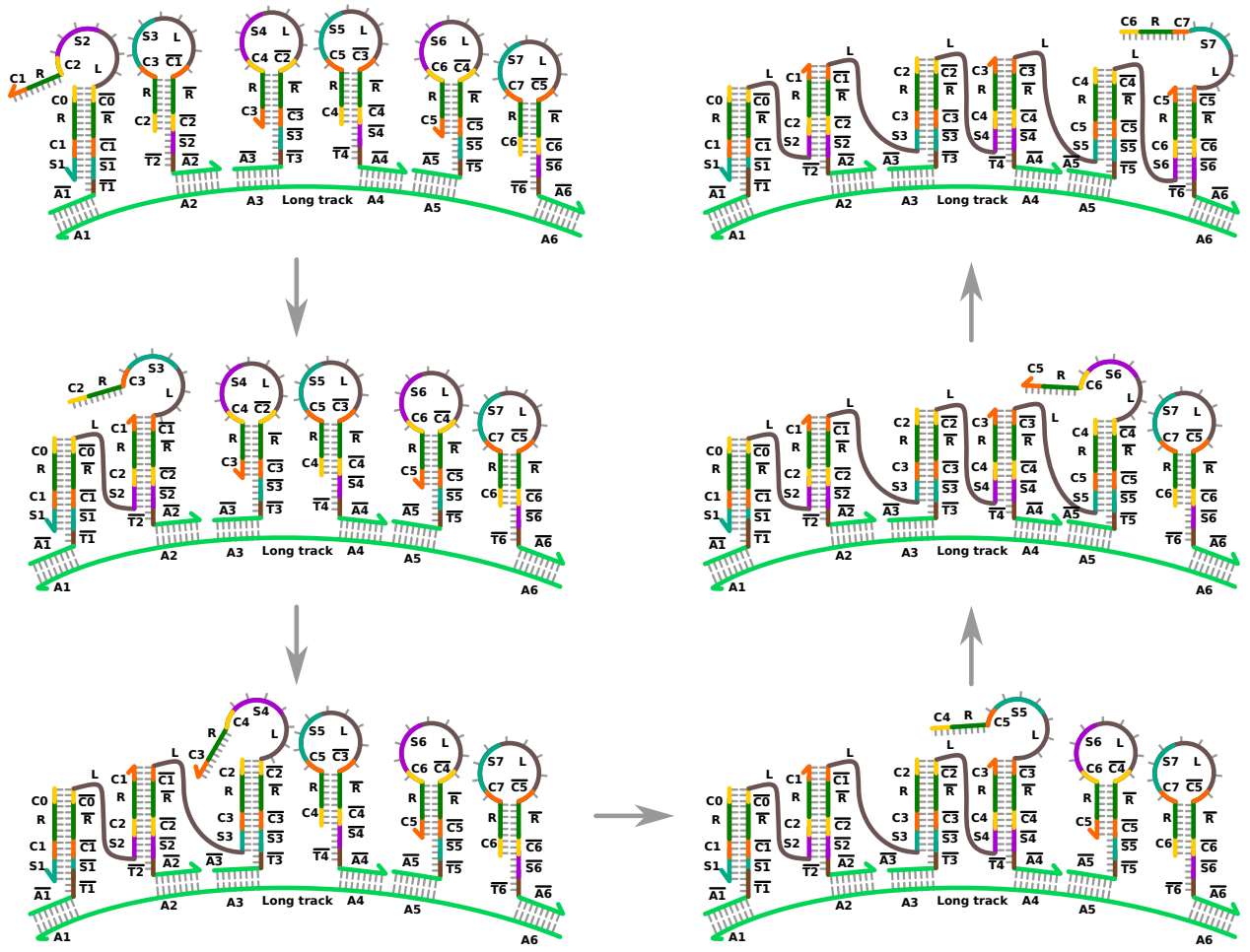

Figure 3.3: Intermediate states of the localized DNA hybridization chain reaction system. After initiation (top-left), the reaction reaches the completion when all hairpins are hybridized (top-right).

\subsection{Mechanism of Localized DNA Hybridization Reactions}

The mechanism of triggering localized DNA hybridization reactions is based on the assumption that all metastable DNA hairpins are successfully attached to the long DNA track. In addition, DNA hairpins are programmed to undergo cascade chain reactions such that the first hairpin only hybridizes to the adjacent hairpin and this process continues until the last hairpin is hybridized. Initially, all hairpin components are anchored to the long track but do not hybridize. The localized DNA hybridization chain reaction occurs when the 
initiator (I) is introduced. The initiator hybridizes to the external toehold $\left(\mathrm{S}_{1}\right)$ of $\mathrm{H}_{1}$ and displaces the stem of $\mathrm{H}_{1}$ by branch migration, opening the loop and revealing its sequestered toehold domain $\left(\mathrm{S}_{2}\right)$ as illustrated in Figure 3.2B (left). The opened loop of $\mathrm{H}_{1}$ can now hybridize to the external toehold of $\mathrm{H}_{2}$ and displace the stem of $\mathrm{H}_{2}$ by branch migration, opening the loop of $\mathrm{H}_{2}$. A similar mechanism occurs until the stem of $\mathrm{H}_{6}$ is displaced by the opened loop of $\mathrm{H}_{5}$ as shown in Figure 3.2B (right). The entire localized cascade DNA hybridization chain reaction occurs on the long DNA track, thus the hairpins are readily available once triggered by the initiator and the kinetic rate is expected to happen faster than in the absence of the long DNA track. The intended reaction pathway of the proposed system is illustrated in Figure 3.3. The use of hairpins enables active components to be physically attached to the surface during hybridization and the likelihood of losing active components is significantly lower than using regular strands from prior studies. ${ }^{112,119,120}$

\subsection{Gel Electrophoresis Method and Ensemble Fluorescence Spectroscopy}

All DNA strands were purchased from Integrated DNA Technologies and purified using the PAGE purification method. A list of all DNA strands is shown in Table 3.1. Native PAGE and agarose gel were used to observe the 
thermodynamic equilibrium of localized DNA hybridization chain reactions. Ensemble fluorescence spectroscopy was used to monitor the real time reaction kinetics of DNA hybridization reactions in the presence and absence of the DNA track. A reporter complex, consisting of a fluorophore (Tetrachlorofluorescein) and a quencher molecule (Iowablack), was able to bind and give rise to fluorescence emission only when the stem and loop of hairpin $\mathrm{H}_{6}$ were opened, depicted in Figure 3.2C. An equimolar solution of hairpins was mixed together. For the hairpin solution with the long DNA track, the solution was quickly heated at $90{ }^{\circ} \mathrm{C}$ for 10 minutes and was let to cool down to room temperature prior fluorescence kinetic experiments. The fluorophore was excited at $524 \mathrm{~nm}$ and the emission measured at $541 \mathrm{~nm}$. The excitation and emission slits were set at $10 \mathrm{~nm}$ and $5 \mathrm{~nm}$, respectively. For localized DNA hybridization reactions, 100 $\mu \mathrm{L}$ of $30 \mathrm{nM}$ sample was filled into a cuvette. All measurements were performed at $25{ }^{\circ} \mathrm{C}$. For kinetic measurements, $1-10 \mu \mathrm{L}$ of initiator strand were added to a $100 \mu \mathrm{L}$ sample. For non-localized DNA hybridization reactions, $80 \mu \mathrm{L}$ of $5 \mathrm{nM}$ sample was used. Rapid mixing was achieved by carefully but quickly pipetting the whole volume up and down for a minute without generation of air bubbles 
or loss of material. All experiments were performed in 1x TAE $\mathrm{Mg}^{2+}$ buffer solution.

Table 3.1: DNA oligomers to assemble linear cascade DNA hybridization chain reactions on the long DNA track. In hairpin sequences, stems are underlined. For kinetic studies, the reporter complex (RC) is displaced hairpin $\mathrm{H}_{6}$.

\begin{tabular}{|c|c|c|}
\hline Name & Sequence (5' to 3') & Length \\
\hline Hairpin $\mathrm{H}_{1}$ & $\begin{array}{l}\text { A1 5'- } \\
\text { GGGTGGGTTGTGATGTATGTAATGTTTTCCTCCTACTCACATAC } \\
\text { ATCACA } \overline{A C-3},\end{array}$ & 52 \\
\hline Hairpin $\mathrm{H}_{2}$ & $\begin{array}{l}5^{\prime} \\
\text { ACTCACATACATCAATCGTCTTTCTTTTGTTGTGATGTATGTGAG } \\
\text { TAGGAGG - 3' A2 }\end{array}$ & 52 \\
\hline Hairpin $\mathrm{H}_{3}$ & $\begin{array}{l}\text { A3 5'- } \\
\text { GAAAGACGATTGATGTATGTGAGTTTTTTCTTCCTTTAACATAC } \\
\text { ATCAATCG -3, }\end{array}$ & 52 \\
\hline Hairpin $\mathrm{H}_{4}$ & $\begin{array}{l}5^{\prime}- \\
\text { TTTAACATACATCACCACTTTCCTTTTTCGATTGATGTATGTTAA } \\
\text { AGGAAGA - 3' A4 }\end{array}$ & 52 \\
\hline Hairpin $\mathrm{H}_{5}$ & $\begin{array}{l}\text { A5 5'- } \\
\text { AGGAAAGTGGTGATGTATGTTAAATTTTCTTCTCCTCCACATAC } \\
\text { ATCACCAC }-3\end{array}$ & 52 \\
\hline Hairpin $\mathrm{H}_{6}$ & $\begin{array}{l}\text { ' - } \\
\text { CTCCACATACATCATATTCCCTCATTCAAGCGTGGTGATGTATG } \\
\text { TGGAGGAGAAG - 3, A6 }\end{array}$ & 55 \\
\hline Initiator I & 5' - CATTACATACATCACAACCCACCC - 3' & 24 \\
\hline RC FQ & 5' - TTGAATGAGGGAATATGATGTATGTGG/Quencher/ - 3' & 27 \\
\hline RC TET & $5^{\prime}$ - /Fluorophore/CCACATACATCATATTCCCT - 3' & 20 \\
\hline Anchor $A_{1}$ & 5' - TTGAGAGTAGTGGAGGAGAGGAGA - H1 & 24 \\
\hline Anchor $\mathrm{A}_{2}$ & H2 - AGAAGGAAGGAAAGGAAAGAAGGA - $3^{\prime}$ & 24 \\
\hline Anchor $\mathrm{A}_{3}$ & $5^{\prime}$ - TTGGTGTGGTGTGGTGTGGTGGTG - H3 & 24 \\
\hline Anchor $\mathrm{A}_{4}$ & H4 - GGAGGAGGAGAAGGAGAGGAGAGA - 3' & 24 \\
\hline Anchor $\mathrm{A}_{5}$ & 5' - GAAGTGAAGAAGAAGAAAGAGAAG - H5 & 24 \\
\hline Anchor $\mathrm{A}_{6}$ & H6 - GAAGAAAGAAGAAGAAGAGAGAAA - 3' & 24 \\
\hline Track LT & 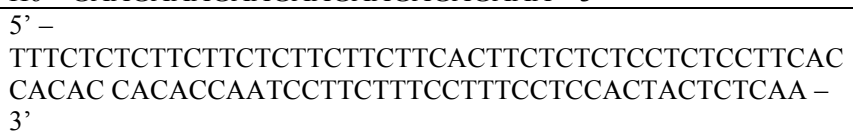 & 90 \\
\hline
\end{tabular}

\subsection{Thermal Equilibrium Characterization}

The thermal equilibrium conditions of the proposed system were first studied by gel electrophoresis. In particular, DNA hairpin sequences were synthesized without the anchor domains. The self-assembly of those hairpins 
was analyzed using 10\% native polyacrylamide gel electrophoresis (PAGE) as illustrated in Figure 3.4 (left panel). Lane 1 contains a DNA marker with 20-bp increments. Lanes 2 to 7 contain a mixture of 1, 2, 3, 4, 5, and 6 hairpin(s) in the absence of the initiator. Products of low molecular weight are visible around 20 $40 \mathrm{bp}$, indicating that individual hairpins remain intact although there is a slightly evidence of crosstalk (undesired reactions in the absence of the initiator) in lanes 4 to 7 around $100-300 \mathrm{bp}$. Lanes 8 to 13 contain a mixture of 1, 2, 3, 4, 5, and 6 hairpin(s) in the presence of the initiator. Products of increasing higher molecular weight are visible and are indicated as marker maroon boxes. In addition, individual hairpin strands were mostly consumed by the cascade reactions. These controls indicate that the oligomers are forming due to the addition of the initiator with the expected lengths of approximately $40 \mathrm{bp}, 80 \mathrm{bp}$, $120 \mathrm{bp}, 160 \mathrm{bp}, 200 \mathrm{bp}$, and $240 \mathrm{bp}$. Presence of residual hairpins of around 20 $40 \mathrm{bp}$ in lanes 8 to 13 could be due to stoichiometric concentration differences between DNA strands, improper synthesized DNA strands that did not participate in the reaction, or excess initiator strand. The successively higher molecular weight products from these controls indicate that DNA hairpins were 
programmed correctly and that the cascade hybridization reaction mechanism was well designed. 


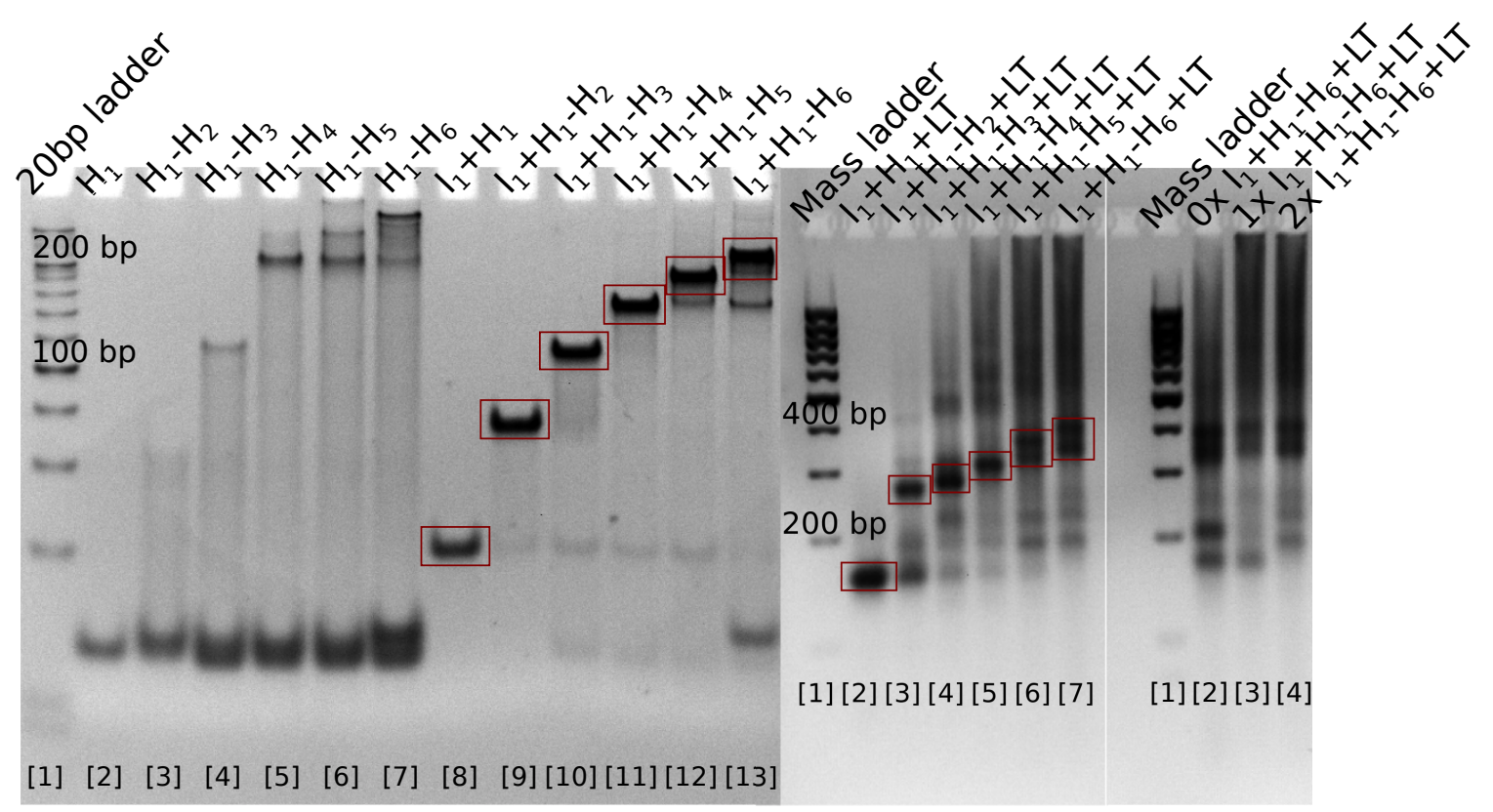

Figure 3.4: (Left panel) 10\% native PAGE gel analysis of HCR: All samples were incubated at room temperature for 200 minutes. All samples were prepared at $500 \mathrm{nM}$ and $34 \mu \mathrm{L}$ of sample was loaded into each lane. All hairpins do not carry the anchor domains. Lane 1 contains DNA marker with 20-bp increments $(20-300 \mathrm{bp})$. Lanes 2 to 7 contain mixture of different numbers of hairpins in the absence of the initiator. Lanes 8 to 13 contain the same corresponding mixture of hairpins in the presence of the initiator. (Middle and right panels) $3 \%$ agarose gel analysis of localized DNA hybridization chain reaction (LCR). All hairpins are conjugated with the anchor domains to self-assemble to the long DNA track. All samples were quickly annealed for 10 minutes and incubated with the initiator for 200 minutes at room temperature. All samples were prepared at $500 \mathrm{nM}$ and $10 \mu \mathrm{L}$ of sample was loaded into each lane. (Middle panel) Step-by-step LCR $500 \mathrm{nM}$ of hairpins were mixed with $2 \mathrm{x}$ LT in the presence of $2 \mathrm{x}$ initiators (I). (Right panel) Effect of initiator concentration to LCR - $500 \mathrm{nM}$ of hairpins were mixed with $2 x \mathrm{LT}$ in the presence of the initiator. Note that the marker maroon boxes indicate major bands of the desired DNA structures. 
To observe the thermal equilibrium conditions of DNA hairpins bound to the long DNA track, DNA hairpin strands were synthesized with the anchor domains. Due to the addition of the long DNA track, the size of DNA hairpins bound to the track was larger than the porous size of 10\% PAGE gel (data not shown). Thus the self-assembly of DNA hairpins on the track was analyzed using 3\% agarose gel electrophoresis as shown in Figure 3.4 (middle panel). Lane 1 contains a DNA marker with 100-bp increments. Lanes 2 to 7 contain a mixture of $1,2,3,4,5$, and 6 hairpins self-assembled on the track prior to the addition of the initiator. Individual hairpins hybridized and bound to the long DNA track as indicated with marker maroon boxes. The successively higher molecular weight products from these lanes indicate that the reactions occurred and resulted in higher products around $200-400 \mathrm{bp}$. Presence of residual bands below the marker boxes in lanes 2 to 7 could be due to stoichiometric concentration differences between DNA strands, or improper synthesized DNA strands. There is a broad distribution of product size above $400 \mathrm{bp}$ in lanes 4 to 7 , indicating a gradual increase in the population of multimers. These multimers could be further minimized by carefully adjusting the stoichiometry between DNA sequences or by tuning the annealing temperature. 
$3 \%$ agarose gel was again used to study the effect of initiator concentration on the localized DNA hybridization reaction of DNA hairpins bound to the track as shown in Figure 3.4 (right panel). Lane 1 contains a DNA marker with 100-bp increments. Lane 2 contains a mixture of 6 hairpins selfassembled on the track in the absence of the initiator: the designed assembly is clearly visible at around $300-400 \mathrm{bp}$; however, there are still hairpin remains below $200 \mathrm{bp}$ marker. Lanes 3 and 4 contain a mixture of 6 hairpins selfassembled on the track in the presence of $1 \mathrm{x}$ and $2 \mathrm{x}$ initiator concentrations, respectively. The designed assemblies are visible in both lanes and at the same location around $300-400 \mathrm{bp}$. Inferring from the intensities in all lanes, the broad distribution of multimers above $400 \mathrm{bp}$ marker appears stronger in the presence of the initiator (lanes 3 and 4) than in the absence of the initiator (lane 2), indicating localized reactions occurred due to the presence of the track.

\subsection{Real Time Kinetic Characterization}

To observe the kinetics of localized DNA hybridization chain reaction of DNA hairpins bound to the long DNA track, a fluorescence-based method was implemented. In particular, an independent reporter complex was designed with a pair of fluorophore and quencher molecules (Figure 3.2C). The reporter 
complex was programmed to only bind to the LCR system after the cascade reaction was completed. Figure 3.5 shows the fluorescence kinetic response of six metastable DNA hairpins triggered by 2 times excess initiators. These hairpin strands were synthesized with the anchor domains. The blue curves represent the fluorescence response of six DNA hairpins in the absence of the long DNA track. The red curves are fluorescence responses of six DNA hairpins in the presence of the track. The fluorescence data indicate that the localized DNA hybridization reaction occurred rapidly compared to the non-localized DNA hybridization reaction. Without the presence of the initiator, the minimal fluorescence signal was observed in both systems (inset, Figure 3.5A). 


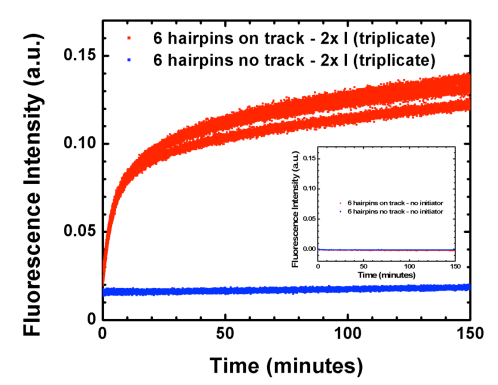

(A)

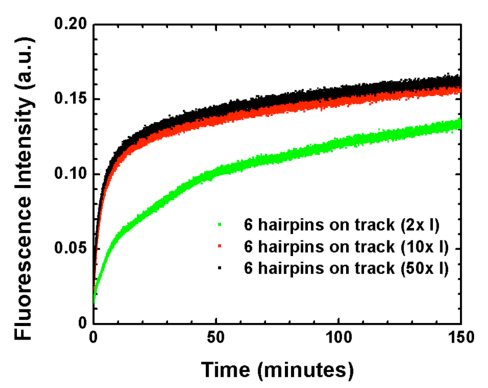

(B)

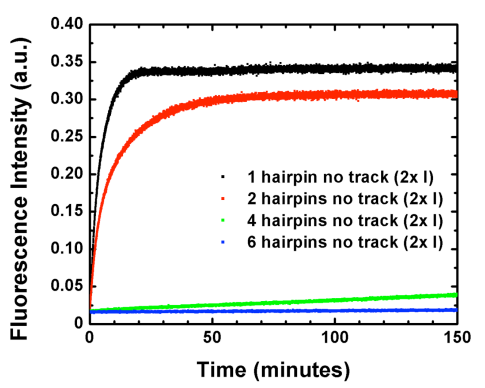

(C)

Figure 3.5: Kinetic characterization of triggered self-assembly hybridization chain reactions. (A) Localized DNA hybridization chain reactions with and without the presence of the long DNA track in 2 times excess initiators. Note: inset shows the fluorescence responses of the same systems but in the absence of the initiator. (B) Effect of localized DNA hybridization chain reaction rate as a function of initiator concentration. (C) Control kinetic analysis of reactions as a function of hairpins in the absence of long DNA track.

To study the effect of initiator concentration on the cascade localized DNA hybridization reactions, various initiator concentrations were added to the same sample consisting of six DNA hairpins bound to the long DNA track as shown in Figure 3.5B. The fluorescence results indicate that insignificant changes are achieved in the presence of more than 10 times excess initiator. The saturation of the rate at 10-50x initiator might also indicate that the interaction between the last hairpin and the quencher-labeled DNA strand is becoming rate-limiting. This means that the localized reaction could be accelerated if the fluorescence reporting mechanism was a part of the last hairpin. In fact, we attempted to test a similar system in which the last hairpin was labeled with a fluorescence purine 
base (2-aminopurine) in order to overcome the rate-limiting factor from using the independent reporter complex. The fluorescence emission result was significantly less pronounced for the system with the concentration below $1 \mu \mathrm{M}$ (data not shown). As mentioned earlier, one of the benefits of exploring localized DNA hybridization reactions is to speed up the reaction at low concentration to avoid leaks (unintended reactions in the absence of the input strand). Other approaches such as i) labeling the last hairpin with the reporter complex which could potentially alter the kinetics of the hairpin structure or ii) using excessive amount of the independent reporter complex to bias the bimolecular process to the pseudo-unimolecular process, could be explored.

To observe the effect of the anchor domains on the overall cascade reaction rate, different samples of DNA hairpins were synthesized in the absence of the long DNA track as shown in Figure 3.5C. Upon adding the initiator, the fluorescence emission increases rapidly up to 20 minutes for the sample with a single hairpin (black curve) and 40 minutes for the sample with a pair of hairpins (red curve). Both samples slowly increase emission as thermal equilibrium was achieved. However, the fluorescence emission occurs slowly for the sample with 4 hairpins (green curve) and for the sample with 6 hairpins (blue curve). These 
results indicate that the reaction rates are slow and never reach thermal equilibrium within the experimental time scale ( 1000 minutes). As indicated by the leftmost gel data in Figure 3.4, the cascade reaction reached thermal equilibrium within 200 minutes for DNA hairpins which were synthesized without the anchor domains. Thus the addition of the anchor domain is possibly the reason that such minimal fluorescence was observed for systems with more than 4 hairpins in the absence of the long DNA track (blue curves, Figure 3.5A). To further demonstrate the effect of the anchor domain, the identical DNA hairpin system (blue curve, Figure 3.5C) was synthesized without the anchor domain. Multiple fluorescence experiments indicate that the designed cascade reaction occurred as expected and that the fluorescence emission was pronounced within the experimental time scale as illustrated in Figure 3.6A. An addition of an anchor domain to assist each DNA hairpin hybridizing to the long DNA track clearly affects the reaction kinetics. 


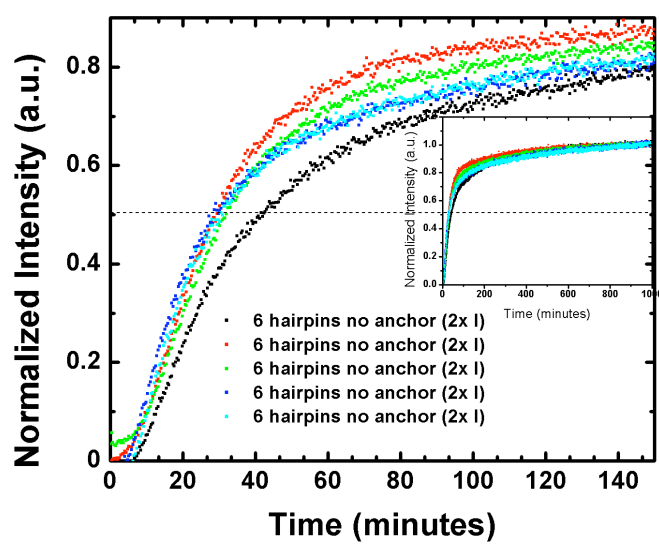

(A)

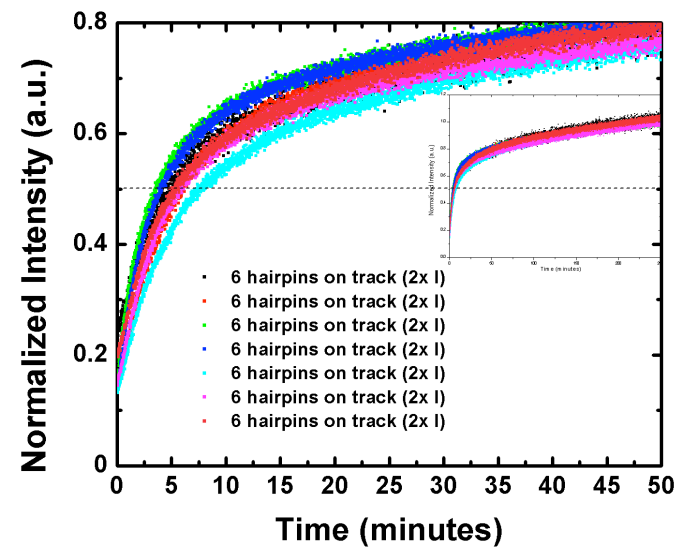

(B)

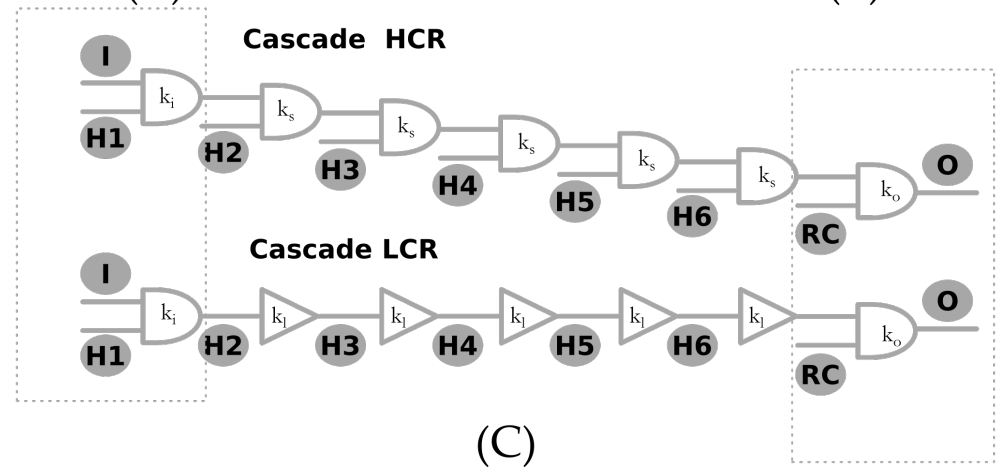

Figure 3.6: Reproducible fluorescence experimental results of the two systems which are then used to quantify the speed-up from tethering DNA hairpins on the long DNA track: (A) six DNA hairpins (no anchor domain, no track) in the presence of $2 x$ initiators, (B) six DNA hairpins bound to the long DNA track in the presence of $2 x$ initiators. Note: Dashed lines indicate half-time completion the time required for the reaction to reach $50 \%$ completion. (C) Model to describe cascade reactions with and without the locality effect. Note: AND symbol indicates bimolecular reaction; TRIANGLE symbol indicates unimolecular reaction.

\subsection{Speed-up from tethering DNA hairpins to the surface}

To establish a systematic approach to measure the speed-up from tethering DNA hairpins on the long DNA track, multiple fluorescence 
experiments were performed for two samples - one sample was synthesized with six DNA hairpins bound the long DNA track, the other sample was synthesized with six DNA hairpins without the anchor domains. All fluorescence experiments were normalized to unity which corresponds to thermal equilibrium. Figure 3.6A and Figure 3.6B show reproducible fluorescence experimental results for the two samples. In particular, fluorescence results for six DNA hairpins (no anchor domain, no track) in the presence of $2 x$ excess initiators are shown in Figure 3.6A. Fluorescence results for six DNA hairpins bound to the long DNA track in the presence of 2x excess initiators are shown in Figure 3.6B. Note that the fluorescence experimental data in Figure 3.6B are the same results as Figure 3.5B but in replicate.

To quantify the speed-up resulting from tethering DNA hairpins to the long DNA track, we first describe the designated reaction pathways for the cascade DNA hybridization chain reactions of six hairpin gates in the presence and absence of the long track (illustrated in Figure 3.6C). In both cases, the initiation and the reporting mechanisms depend on the diffusion rates $\left(k_{i}\right.$ and $\left.k_{o}\right)$ of the initiator to the first hairpin gate and of the last hairpin to the reporter complex; these two processes are better described as bimolecular reactions 
(dashed boxes). In the absence of the track, the cascade hybridization chain reaction of six hairpins is treated as a bimolecular reaction, and because all the hairpins have a similar structure, they are assumed to have the same rate constant $\left(k_{s}\right)$. In the case of six hairpin gates bound to the track, the localized cascade hybridization chain reaction is treated as a unimolecular reaction and all the hairpins are assumed to interact with the same rate constant $\left(k_{l}\right)$. It is worth noting that the rate constant for the bimolecular reaction depends on the concentration of individual hairpins. In contrast, the rate constant for the unimolecular reaction is independent of the concentration of individual hairpins assuming all hairpins are assembled correctly on the track substrate. For our systems, the ensemble rate constant is a superposition of either multiple bimolecular processes (cascade HCR) or multiple unimolecular and bimolecular processes (cascade LCR). A reasonable quantity that can be used to measure the speed-up factor is the half-time completion for both systems as illustrated by dashed lines in Figure 3.6A and Figure 3.6B. The half-time completion of the cascade DNA hybridization chain reactions of six hairpins was determined to be 32.77 minutes, indicating that half of the hairpin population was consumed in the reaction. The half-time completion of the localized DNA hybridization chain 
reactions of six hairpins was determined to be 5.31 minutes. Independent of the multiple rate constants that dictated our systems, the empirical data indicate that the localized reaction experiences a factor of six times speed-up compared to the non-localized reaction.

To estimate the ensemble rate constants for our systems, we further assumed both systems experience the same kinetic effect arising from initiation and termination. Hence, the ensemble rate constant is inversely proportional to the half-time completion of the reaction. For the bimolecular reaction, the reaction rate depends on the initial concentrations of hairpins. For the unimolecular reaction, the reaction rate is independent of the initial concentrations of hairpins given that all hairpins correctly self-assembled on the track. The ensemble rate constant for cascade HCR was determined to be $1.017 \mathrm{x}$ $10^{5} \mathrm{M}^{-1} \mathrm{~s}^{-1}$. The ensemble rate constant for cascade LCR was determined to be 2.2 $x 10^{-3} \mathrm{~s}^{-1}$. Because the likelihood of having all hairpins attached to the long DNA track critically depends on the exact stoichiometry mixing, the assembly yield of the cascade LCR is expected to be harder to control than that of the cascade HCR. Although DNA hairpins bound to the long DNA track show evidence of cascade LCR, the distances between those hairpins have yet to be explored. Thermal 
equilibrium gel data and real-time fluorescence kinetic results indicate that the localized DNA hybridization reaction was clearly enhanced via tethering DNA hairpins to the long DNA track. For unlimited HCR with the assembly of multiple hairpin mononers, a larger surface area such as DNA origami could be employed. In particular, DNA origami rectangle designed by Rothemund ${ }^{15}$ could accommodate up to 200 distinct addressable sites. In addition, the proposed system can be extended to form cyclic reactions by modifying the last hairpin to interact with the first hairpin which allows for the creation of longer oligomers.

\subsection{Conclusion}

With the goal of increasing the reaction rates, we experimentally demonstrated a surface-bound method by tethering hairpins to a long DNA track. The anchor domains which link the hairpins to the long DNA track have been shown to affect the reaction kinetics via fluorescence kinetic studies. The rate-limiting factor could be due to the independent reporter complex. We demonstrated an overall six times speed-up of localized DNA reactions over non-localized DNA reactions. It is important to note that this is an overall speedup and not purely due to the localized hybridization reaction. The actual localized rate constant between a pair of hairpins still remains a challenge. Thus 
a new model for determining the localized rate constant is needed to fully measure the actual speed-up of the localized hybridization reactions. The proposed system provides one of the first known quantifications of the speed-up of DNA hybridization reactions due to the locality effect. One possible approach to measure the localized rate constant is to tether the initiator to the surface and to label the last hairpin with the reporter complex. Ideally the initiation could be triggered by irradiation and the termination could be measured by the fluorescence emission from the last hairpin. This approach could possibly overcome the rate-limiting factor due to the initiation and the termination. In addition, the localized hybridization chain reaction on the surface of DNA origami with a larger number of DNA hairpins could be explored. We conjecture that if we extend our experiments to have more than 6 hairpins on DNA origami, we will experience a much larger speed-up over non-localized hybridization reactions. We envision and are currently exploring the construction of Boolean logic, such as AND and OR gates, on the surface of DNA nanostructures. Recent advances in vitro evolution enabled the capability to generate a set of DNA aptamers that have a strong binding affinity to surface-bound proteins. Our proposed localized DNA hybridization reaction may be of practical use in 
performing surface computation on cellular membranes for disease detection and prevention. 


\section{Localized DNA Hybridization Chain Reactions on Large DNA Nanostructures}

Given the success of operating DNA hybridization reactions on the surface of DNA nanotrack, the next step is to further demonstrate the possibility of operating DNA hybridization reactions on a much larger surface since the track provides a limited number of binding sites for tethering DNA hairpin gates. This chapter will investigate DNA hybridization reactions on the surface of DNA origami rectangle with the potential to accommodate up to 200 DNA hairpin gates. The majority of this work has been submitted for journal publication as: Hieu Bui, Shalin Shah, Reem Mokhtar, Sudhanshu Garg, Tianqi Song, and John Reif, "Localized DNA hybridization chain reactions on DNA origami".

\subsection{Background}

The field of DNA nanoscience has demonstrated many exquisite DNA nanostructures and intricate DNA nanodevices. However the operation of each step of prior demonstrated DNA nanodevices has generally required the diffusion of DNA stands, and the resulting diffusion kinetics has limited the speed of these devices. Here we demonstrate chains of localized DNA hybridization reactions on the surface of a self-assembled DNA origami rectangle. The localization design for our DNA nanodevices is novel in that it 
avoids the diffusion of DNA strands on each step, thus providing faster reaction kinetics. The locality also provides considerable increased scalability, since localized components of the devices can be reused at other locations. A variety of techniques, including AFM, TIRF, and ensemble fluorescence spectroscopy are used to confirm the localized DNA hybridization reactions on the surface of DNA origami. There are many potential cellular and biological applications for our localized DNA nanodevices, and moreover the localization design lends itself to applications involving DNA nanodevices operating on other molecular surfaces, such as cellular surfaces.

\subsection{Overview DNA Nanoscience}

Self-assembly, programmability, and predictability are the major properties of DNA. Since the first demonstration of creating lattices from a finite set of DNA sequences ${ }^{14}$, many exquisite nanoscale structures and intricate devices have been reported ${ }^{12,13,24,26,49-52,57,58,125}$. There are two major research themes concurrently being explored within the field of DNA nanotechnology. First is called structural DNA nanotechnology where molecular self-assembly via hybridization reactions creates nanoscale molecules that can be expanded to microscale structures ${ }^{24}$. The other is called dynamic DNA nanotechnology where 
programmable dynamic nanomachines are used to produce motions and perform (primitive) computations ${ }^{26}$.

There are multiple exciting potential cellular and biological applications ${ }^{53,126,127}$ of dynamic DNA nanodevices. For instance, the stability of DNA nanostructures in the cellular environment has been explored in multiple studies $^{128-130}$. In addition, DNA nanostructures were designed as intelligent nanomachines to carry RNA $^{131}$, aptamers ${ }^{132}$, vaccines ${ }^{133}$ and drugs ${ }^{134}$ to target cellular structures. The ability to perform primitive computation on cellular surfaces may provide the following benefits: i) the output signal can be released by a correct set of chemical inputs for detection and sensing, ii) the presence of multiple extracellular proteins can be observed via a simple set of DNA-based Boolean logic gates, and iii) the mechanism of cell entry for nanostructures can be investigated via integration of responsive input molecules to the extracellular and intracellular environments. However, DNA hybridization reactions on cellular surfaces have not been extensively investigated.

A feasible first step in this direction is to develop and refine the capability of localized DNA hybridization reactions on self-assembled DNA nanostructures. This effort would join the two research fields (structural and 
dynamic DNA nanotechnology) to fully exploit the potential of DNA nanotechnology. In particular, there have recently been some investigations of localized DNA hybridization reactions on DNA origami nanostructures ${ }^{15}$. For instance, Teichmann et al. studied the effect of colocalization by immobilizing a two-stage DNA strand displacement reaction onto a DNA origami platform ${ }^{119}$. Kopperger et al. studied the diffusive transport of DNA cargo strands bound to a DNA origami structure ${ }^{112}$. Ruiz et al. constructed a simple see-saw DNA amplifier by tethering DNA strands to DNA origamis ${ }^{120}$. Several theoretical works have shown that constraining distances among DNA strands lead to faster reaction rates ${ }^{104,113,135}$. However, all these prior works required the diffusion of DNA strands at each step of their operation, but the diffusion kinetics limits the speed of their operation. Further, the global diffusion of DNA strands on each step of their operation also limits the scalability of the localized systems.

Here we present a system to investigate DNA hybridization reactions on the surface of a DNA origami rectangle as a first step towards performing computation on cellular and other biological surfaces. The proposed system exploits a novel design in order to achieve faster reaction kinetics than regular DNA reactions. This system combines the large surface area of the DNA origami 
nanostructure and the non-equilibrium linear cascade DNA hybridization reactions to deliberately achieve localized signal propagation responses. Unlike prior works on localized DNA hybridization reactions, the proposed system utilizes the unique properties inherent in DNA hairpins instead of regular DNA strands for constructing the linear cascade reaction. In particular, DNA hairpins can be programmed to undergo conformational changes, can be responsive to external stimuli such as DNA strand displacement, and can be designed to withstand the loss of active components during the reaction.

\subsection{Design and mechanism of localized DNA hybridization chain reaction system}

DNA origami rectangle design from Rothemund ${ }^{15}$ is an ideal candidate for studying the proposed localized DNA hybridization reaction because of its versatility and well-established protocol as shown in Figure 4.1. The design consists of 224 short staple strands and a long scaffold M13mp18 (refer to Figure 2 for additional design details). A subset of staple strands was modified with 24nt length sticky ends (green circles in Figure 4.1) to attach DNA metastable hairpins via anchor domains $\left(\mathrm{A}_{\mathrm{i}}\right)$. To study the localized DNA hybridization reaction, a set of distinct metastable DNA hairpins was designed to i) precisely bind to the surface of the DNA origami rectangle and ii) be triggered ${ }^{100}$ and 
undergo a cascade DNA hybridization chain reaction in the presence of an external stimulus. Each metastable DNA hairpin consists of a stem, a loop, and a sticky end. The sticky end consists of a spacer domain $\left(\mathrm{T}_{\mathrm{i}}\right)$, an anchor domain $\left(\mathrm{A}_{\mathrm{i}}\right)$, and an external domain $\left(\mathrm{S}_{\mathrm{i}}\right)$. The purposes of the sticky end are $\left.\mathrm{i}\right)$ to anchor the hairpin to DNA origami surface via $A_{i}$ domain and ii) to encode an external toehold $\left(\mathrm{S}_{\mathrm{i}}\right)$ for the localized DNA hybridization reaction. The stem consists of two domains $\left(C_{i}\right.$ and $\left.R\right)$ to form a stable duplex with 14 base pairs. The purpose of the stem is to sequester active domains $\left(S_{i+1}\right)$ within the loop prior to triggered self-assembly. The loop consists of two clamp domains ( $\mathrm{C}_{\mathrm{i}-1}$ and $\left.\mathrm{C}_{\mathrm{i}+1}\right)$, a linker domain $(\mathrm{L})$, and a sequestered domain $\left(\mathrm{S}_{\mathrm{i}+1}\right)$. The purposes of the loop are i) to encode the sequestered domain for the cascade reaction and ii) to fasten the cargo $\left(\mathrm{S}_{\mathrm{i}+1}\right)$ from flowing away to solution. Each hairpin has two toehold domains $\left(S_{i}\right.$ and $\left.S_{i+1}\right)-S_{i}$ is an external toehold domain and readily available for hybridization and $S_{i+1}$ is an internal toehold domain and unavailable for hybridization. The hairpin motif is similar to the design in the previous chapters with an exception that the anchor domains are intended to hybridize to the modified staple strands of the DNA origami rectangle. An alternative strategy to attach DNA hairpins to DNA origami is to directly synthesize the hairpins to the 
staple strands. Two potential drawbacks from the latter strategy are: i) the intended reaction could be altered during one-pot DNA origami thermal process, and ii) the oligomers could end up to be beyond the standard synthesis length which could hinder the scalability.

The mechanism of the localized DNA hybridization reaction is shown in Figure 4.1B. Initially, all DNA hairpins are self-assembled to the DNA origami rectangle via binding to the anchor domains. Upon adding the initiator, the localized DNA hybridization reaction is initiated. In particular, the initiator hybridizes to the first hairpin $\mathrm{H} 1$ via domain $S_{i}$ and displaces its stem domain, thus activating its sequestered domain $S_{i+1}$ within its loop. The sequestered domain can now hybridize to the adjacent hairpin $\mathrm{H} 2$, resulting in a similar effect. The process continues until the last hairpin H6 is fully opened. Without localization to the DNA origami rectangle surface, the hairpins would be expected to react slower in order to complete the cascade reaction due to diffusion. In addition, since the proposed system can be operated at low concentration, the leaks (undesired reactions in the absence of input sequences) are expected to be lower than at high concentration ${ }^{4}$. 


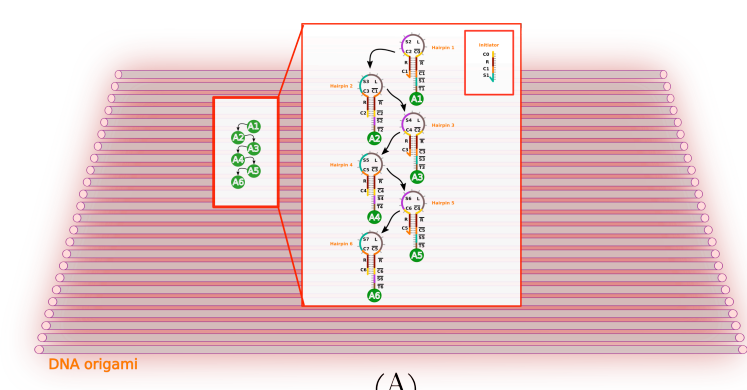

(A)

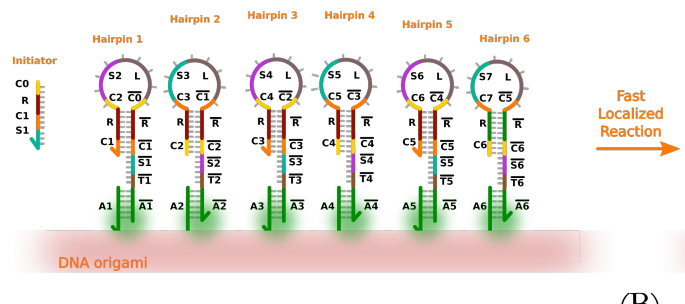

(B)

Figure 4.1: Summary of localized DNA hybridization chain reaction. (A) DNA origami rectangle with modified staple strands (green circles). Metastable DNA hairpins tethering to DNA origami rectangle's surface via modified staple strands through the anchor domains. (B) Mechanism of DNA hybridization reactions on DNA origami surface.

\subsection{Assembly of DNA Origami Rectangle}

DNA origami rectangle was designed using caDNAno ${ }^{25}$. A 7249 nt long

DNA sequence was derived from the genome of the bacteriophage Mp13mp18

and was used as a scaffold as shown in Figure 4.2. Staple strands, extended staple strands and dye-labeled oligonucleotides were purchased from Integrated DNA Technologies, USA. The scaffold M13mp18 was purchased from Guild BioSciences. All sequences and modifications are listed in Table 4.1 and Table 4.2, and Appendix A. DNA hairpins were purified using 10\% denaturing polyacrylamide gel electrophoresis (PAGE) prior to self-assembly to DNA 
origami rectangles. DNA origami structures were folded in a one pot thermal annealing reaction (from $90^{\circ} \mathrm{C}$ to $22^{\circ} \mathrm{C}$ within 90 minutes) at $100 \mathrm{nM}$ scaffold concentration and $1000 \mathrm{nM}$ (10x molar excess over the scaffold) for each structural staple and $5000 \mathrm{nM}$ (20x molar excess) for modified staple strands, which were used for the attachment of the localized DNA hybridization chain reactions. Staples at both ends of the origami structure were left out to prevent blunt-end stacking. The folding buffer contained $12.5 \mathrm{mM}$ magnesium acetate and 1x TAE (40 mM Tris, $20 \mathrm{mM}$ acetic acid, $1 \mathrm{mM}$ EDTA, $\mathrm{pH}$ 8.0) and was also used for further sample preparation. For purification of the folded origami structures (removing excess DNA staple strands) $40 \mu \mathrm{L}$ of DNA origami rectangle mixture at $12.5 \mathrm{mM}$ TAE $\mathrm{Mg}^{2+}$ was mixed with $40 \mu \mathrm{L}$ of $15 \%$ PEG 8000 $(\mathrm{w} / \mathrm{v})$ at $12.5 \mathrm{mM}$ TAE $\mathrm{Mg}^{2+}$. The sample was mixed by tube inversion without generating air bubbles and spun at 13,000g for 25 minutes. The supernatant was pipetted and discarded. The remaining pellet was dissolved in $12.5 \mathrm{mM}$ TAE $\mathrm{Mg}^{2+}$ for 8 hours. After removing excess staple and modified staple strands, a mixture of DNA hairpins was added to DNA origami rectangles with extended staple strands in 4 times excess. The solution was left overnight and 15\% PEG 
$8000(\mathrm{w} / \mathrm{v})$ was performed to remove excess hairpin strands. The PEG purification procedure was done similarly to the step mentioned above.

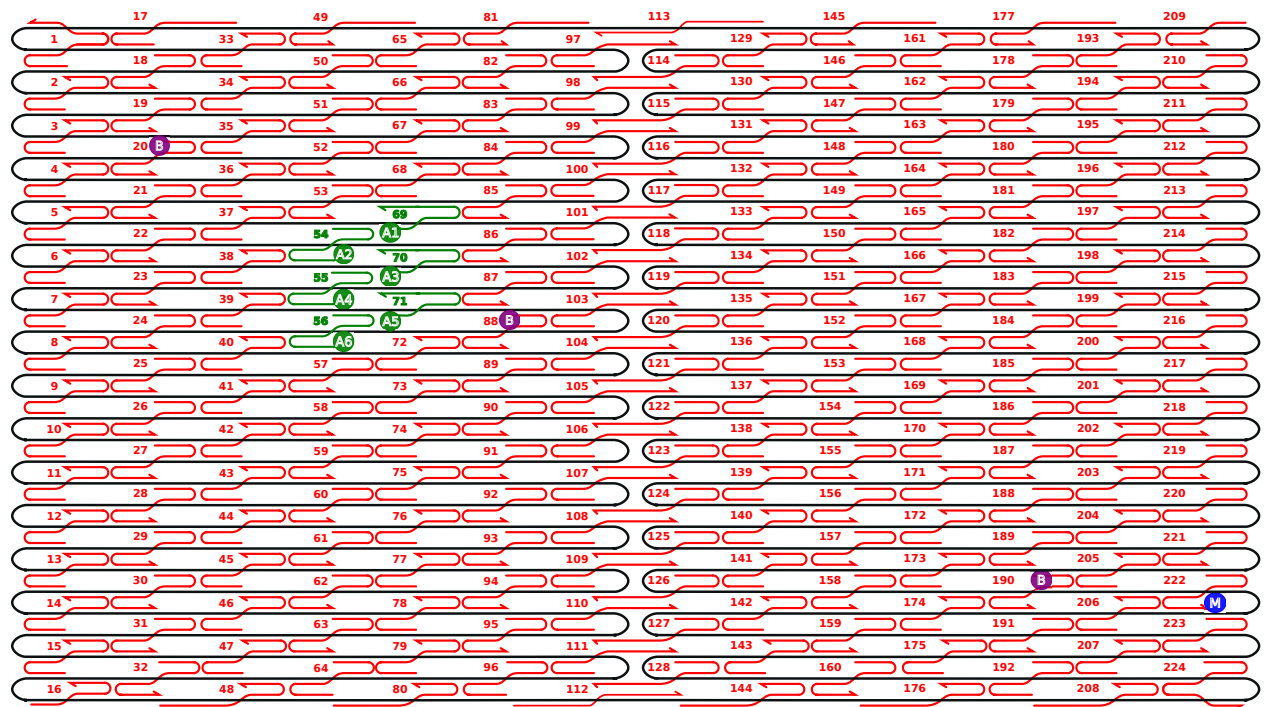

Figure 4.2: DNA origami rectangle design. Staple strands are colored red. Scaffold strand is colored black. Modified staple strands for tethering DNA hairpins are colored green. Biotin-labeled staple strands are colored purple. Modified staple strand for attaching marker sequence is colored blue.

\subsection{Atomic Force Microscopy Imaging Technique}

Imaging of individual origami DNA nanostructures using atomic force

microscopy was carried out with a Bruker Multimode VIII scanning probe microscope. $5 \mu \mathrm{L}$ of nanostructure solution at $5-10 \mathrm{nM}$ were deposited to a freshly cleaved mica surface. After an addition of $20 \mu \mathrm{L}$ of $1 \times \mathrm{TAE} \mathrm{Mg}^{2+}$ buffer, the solution was incubated for 5 minutes at room temperature, then rinsed with $100 \mu \mathrm{L}$ DI water thrice, and dried with nitrogen gas. ScanAsyst mode was used 
to image DNA nanostructures in dry mode using Bruker microcantilever DNP10C probes.

\subsection{Total Internal Reflection Fluorescence Microscopy}

For specific binding of DNA origami rectangles to the glass surface, biotin/streptavidin chemistry was used in order to observe the reaction kinetics. In particular, three staple strands were biotinylated at the 5' end (see Table 4.1). A $35 \mathrm{~mm}$ covered glass slide (MatTek Corporation, USA) was rinsed with $500 \mu \mathrm{L}$ of $1 x$ phosphate-buffered saline (PBS), thrice. After final rinse, $200 \mu \mathrm{L}$ of 0.5 $\mathrm{mg} / \mathrm{mL}$ biotin-labeled bovine serum albumin (BSA) was incubated on the glass slide for 5 minutes. After washing the glass slide, again, with $500 \mu \mathrm{L}$ of $1 \mathrm{x}$ PBS, $200 \mu \mathrm{L}$ of $0.5 \mathrm{mg} / \mathrm{mL}$ streptavidin was incubated on the glass slide for 5 minutes. After removing excess solution from glass slide, $200 \mu \mathrm{L}$ of $1.25 \mathrm{nM}$ DNA origami solution (with 10x excess of all the labeled strands) in 1x PBS was incubated for 10 minutes prior to observation of reaction kinetics under microscope. 1x PBS was used as imaging buffer solution. To perform far-field microscopy, Leica DMI 6000B motorized inverted fluorescence microscopy was used in total internal reflection mode with an objective lens $(x 100, N A=1.46)$ in immersion oil. To collect fluorescence from the sample, Hamamatsu (C9100-13) Electron Multiplying Charge Coupled Device (EM-CCD) was used because of its single 
photon collection capabilities. The cell-size for EM-CCD is $16 \mu \mathrm{m} \times 16 \mu \mathrm{m}$ with the image size of 512 x 512 pixels. By using a 100x objective, a pixel size of approximately $160 \mathrm{~nm}$ was achieved. To excite fluorescent dyes, laser diodes with 488, 561, and $635 \mathrm{~nm}$ wavelength were used. Fluorescence emissions from fluorescent dyes were filtered through the VBG triple filter (Volume Bragg Gratings - x490/20 d435 m465/45|x422/44 d505 m545/55 x552/24 d550 m610/65) and Cy5 filter (x 620/60 d660 m700/75). To minimize photo-bleaching, laser intensity was carefully tuned $(<20 \%$ laser intensity and level 2 from Leica TIRFM).

To quantify TIRFM data, ThunderSTORM plugin from ImageJ software package was employed ${ }^{2}$. The software package was first used to pass TIRFM data through a high pass filter. Then, they were thresholded based on a designated pixel intensity and fitted to a 2D Gaussian curve since point sources are well approximated using the 2D Gaussian curve. All of this processing led to noise free, high-quality images. Further, all the spots which drifted to distances less than $70 \mathrm{~nm}$ (size of origami) were merged together under the assumption that were same spots. Finally, the technique of fiducial markers was used to account for drift in the origami spots. 


\subsection{Ensemble Fluorescence Spectroscopy}

Ensemble fluorescence spectroscopy was used to monitor the kinetics of DNA hybridization reaction in solution and on the surface of the DNA origami. A reporter complex consisting of a fluorophore (TET) and a quencher molecule (FQ) was used to monitor the reaction completion. Only the last hairpin when activated, binds to the reporter complex to trigger the fluorescence emission. An equimolar solution of hairpins was mixed together. The fluorophore was excited at $524 \mathrm{~nm}$ and emission measured at $541 \mathrm{~nm}$. The excitation and emission slits were set at $10 \mathrm{~nm}$ and $5 \mathrm{~nm}$, respectively. For each experiment, $80 \mu \mathrm{L}$ of sample (5 $\mathrm{nM}$ of DNA hairpins, $6.5 \mathrm{nM}$ of reporter complex) was filled into a clean fluorescence cuvette. All measurements were performed at $20^{\circ} \mathrm{C}$. For kinetic measurements, $1-10 \mu \mathrm{L}$ of initiator strand was added to a $80 \mu \mathrm{L}$ sample. Rapid mixing was achieved by carefully but quickly pipetting the whole volume for a minute without generation of air bubbles or loss of material. All experiments were performed in 1x TAE $\mathrm{Mg}^{2+}$ buffer solution.

\subsection{AFM Characterization}

To characterize the self-assembly of DNA hairpins to the surface of a DNA origami rectangle, a solution of DNA origami rectangles mixed with DNA hairpins was imaged using atomic force microscopy (AFM) as shown in Figure 
4.3A. DNA origami rectangles were clearly formed and 6 hairpins formed a height feature complex similar to the designated location as illustrated in Figure 4.1 and Figure 4.2. The initiator was then added to the sample to initiate the localized DNA hybridization reaction. After thermal equilibrium was reached, the solution was then imaged as shown in Figure 4.3B. The resulting assembly of 6 hairpins was expected to form a DNA duplex. Since it is a challenge to observe DNA duplex via AFM, a biotin-labeled output strand was designed to only bind to the last hairpin and the strand was used to detect the completion of the localized DNA hybridization reaction via biotin-streptavidin chemistry. Figure 4.3C shows the result of binding the biotin-labeled output strand to the last hairpin after completion. The biotin-streptavidin complex is present near the location of 6 hairpins, indicating that i) the hairpins were successfully selfassembled to the surface of DNA origami rectangle, and ii) the hairpins underwent localized DNA hybridization reactions after initiation. 


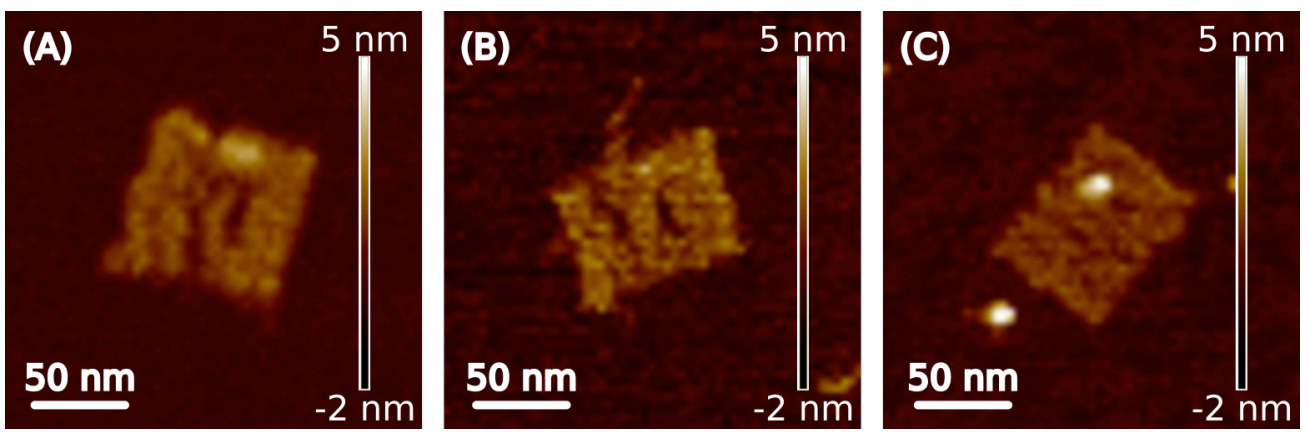

Figure 4.3: AFM characterization of DNA origami rectangle labeled with DNA hairpins at thermal equilibrium. Six metastable DNA hairpins self-assembled on DNA origami rectangle (A), after adding the initiator (B), and after adding the biotin-labeled output to detect the reaction completion (C). Additional data are shown in Figure 4.4 with bigger survey areas.

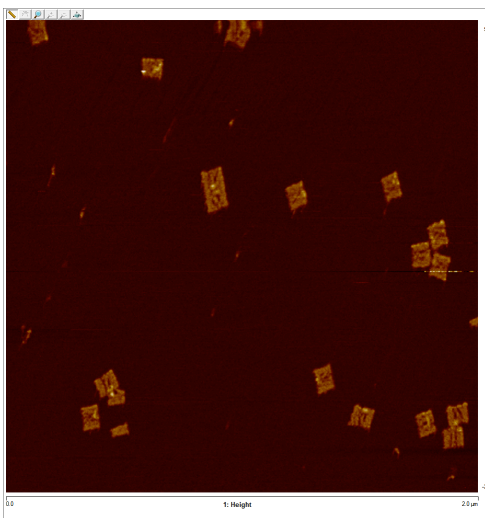

(A)

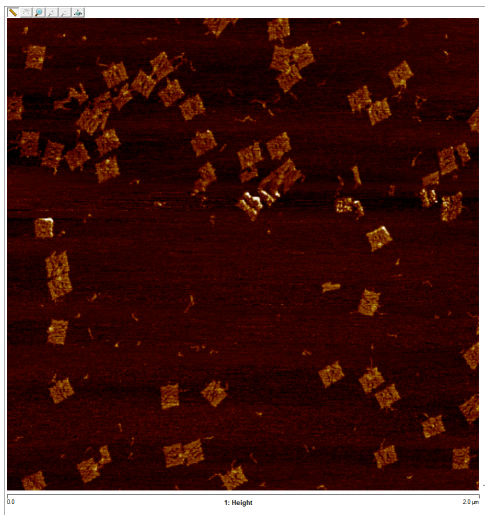

(B)

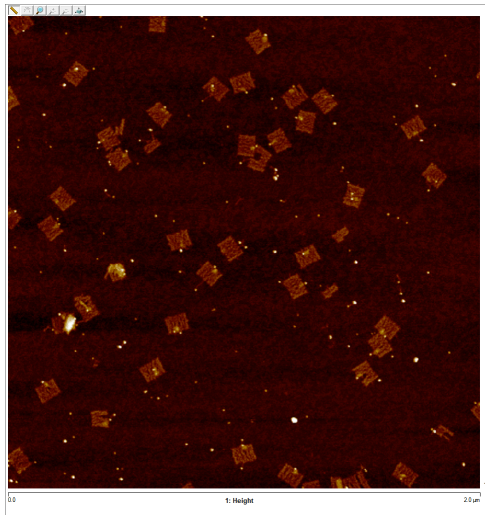

(C)

Figure 4.4: AFM characterization of DNA origami rectangle labeled with DNA hairpins at thermal equilibrium at $2 \mu \mathrm{m}$ scan size. Six metastable DNA hairpins self-assembled on DNA origami rectangle (A), after adding the initiator (B), and after adding the biotin-labeled output to detect the reaction completion $(\mathrm{C})$.

\subsection{TIRFM Characterization}

To observe single molecule reaction kinetics of the localized cascade DNA

hybridization chain reaction, total internal reflection fluorescence microscopy

(TIRFM) was utilized. In general, dye-labeled nanostructures bound to the glass 
surface within the laser evanescent excitation field can be observed as described in the previous section. As shown in Figure 4.5, three distinct fluorescence molecules were used to monitor the reaction kinetics. For identifying the presence of DNA origami rectangles labeled with DNA hairpins, a marker dye was labeled with ATT647N dye (excitation and emission peaks at $649 \mathrm{~nm}$ and $662 \mathrm{~nm}$, respectively). For detecting the initiation of the localized cascade DNA hybridization reaction, the initiator was labeled with TAMRA dye (excitation and emission peaks at 559 and $583 \mathrm{~nm}$, respectively). For detecting the completion of the localized cascade DNA hybridization reaction, an output strand (O6) was labeled with FAM dye (excitation and emission peaks at 495 and $520 \mathrm{~nm}$, respectively). The output strand O6 was designed to only hybridize with the last hairpin when the reaction completed. Prior to TIRFM measurements, 1.25 nM of DNA hairpins bound to DNA origami rectangle solution was incubated with 10x excess of dye-labeled marker strand for 10 minutes. The presence of DNA origami rectangles was visible when the marker dye was excited at $635 \mathrm{~nm}$ (Figure 4.5A). Exciting the sample with $488 \mathrm{~nm}$ and $561 \mathrm{~nm}$ wavelengths resulted in no emission when the initiator and the output strands were not added to the sample. 

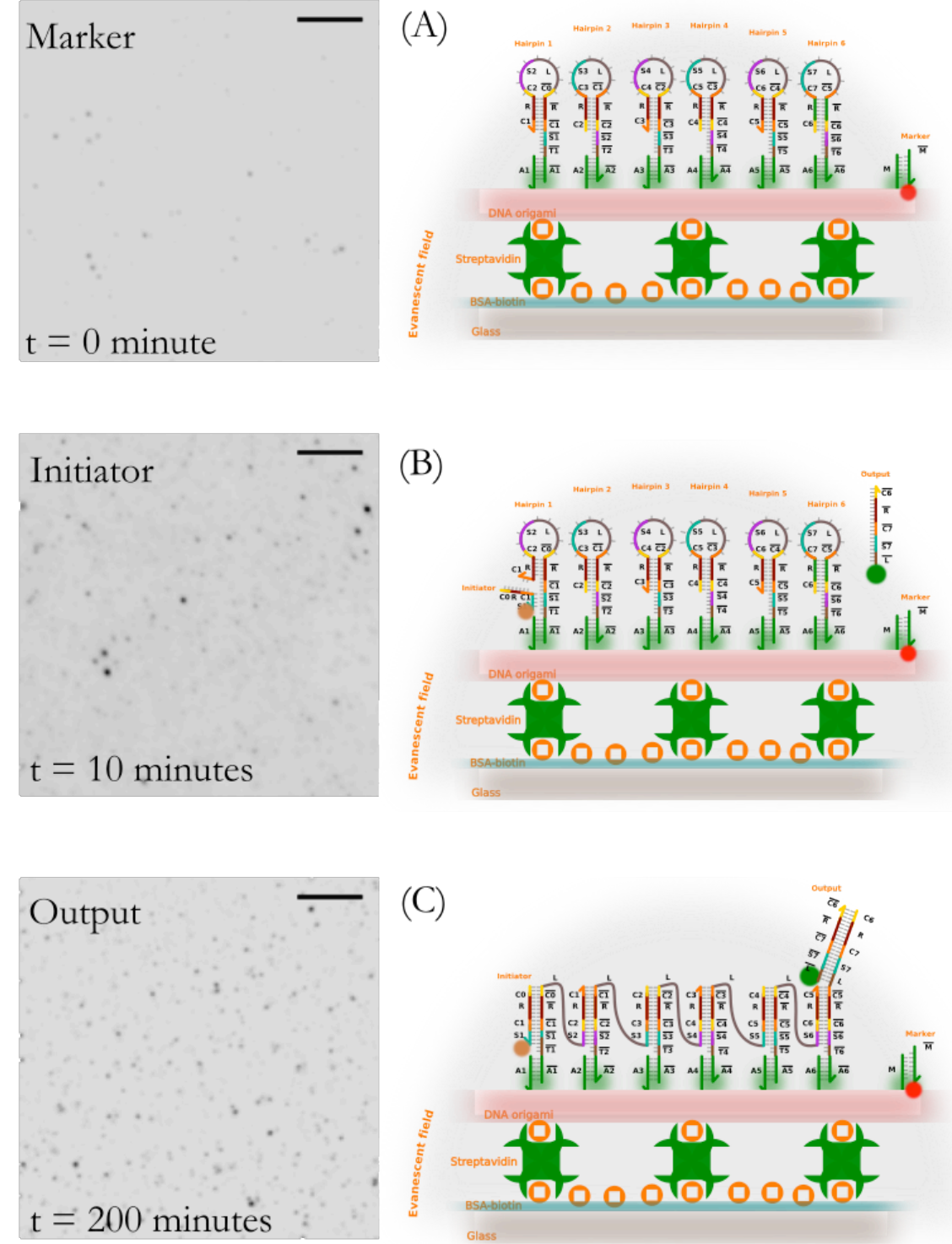

Figure 4.5: Total internal reflection fluorescence characterization (TIRF). TIRF far-field microscopy image of solution containing DNA hairpins on origami before initiation (A), during reaction (B) and after completion (C). Left column is the fluorescence emission of the dye-labeled strand after being excited with a designated excitation wavelength. Right column is the schematic of the dye-labeled strand after being excited with a designated excitation wavelength. All the scale bars are $1 \mu \mathrm{m}$. Additional data are shown in Figure 4.6 with larger survey areas. 

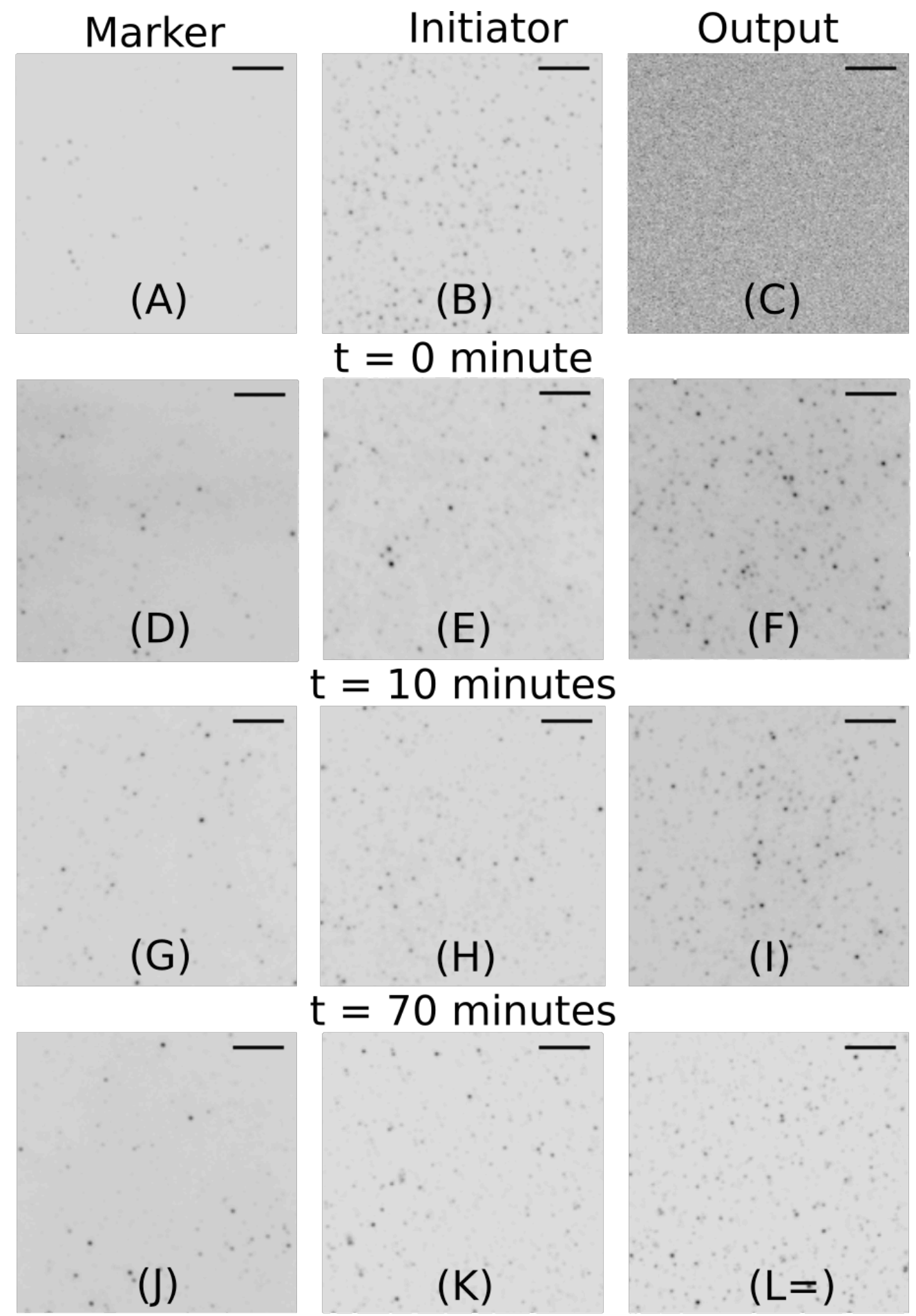

\section{$\mathrm{t}=200$ minutes}

Figure 4.6: Kinetics of localized cascade DNA hybridization reactions overtime via TIRFM. Left column: fluorescence emission of the dye-labeled marker 
strand(A, D, G, J). Middle column: fluorescence emission of the dye-labeled initiator strand (B, E, H, K). Right column: fluorescence emission of the dyelabeled output strand (C, F, I, L). Scale bars are $1 \mu \mathrm{m}$.

After adding the initiator and output strands in 10x excess to the solution of $1.25 \mathrm{nM}$ of DNA hairpins bound to DNA origami rectangle, the sample was incubated for 10 minutes. Afterwards, the presence of DNA origami was detected via exciting the dye-labeled marker strand which bound to the DNA origami rectangle (similar to Figure 4.5A). The initiation of the localized DNA hybridization reaction was observed when the dye-labeled initiator strand was excited at $561 \mathrm{~nm}$ wavelength (Figure 4.5B). After letting the sample react for 200 minutes, the sample was again observed. The completion of the localized DNA hybridization reaction was monitored when the dye-labeled output strand was excited at $488 \mathrm{~nm}$ (Figure 4.5C). Each fluorescence molecule was excited with its corresponding excitation wavelength. The emission from each dye confirms that the dyes were incorporated on the surface of DNA origami, indicating that i) the emission from the marker dye confirms the observed DNA nanostructures adhered to the glass surface and within the laser evanescent field, otherwise the TIRF effect would result in no emission, ii) the emission from the initiator dye confirms the initiation of the localized DNA hybridization reaction, and iii) the emission from the output dye confirms the localized DNA hybridization reaction 
reached completion. Due to the fast localized nature of the reaction, the TIRFM was not able to capture the reaction rate. It remains a challenge to simultaneously monitor the localized reaction via TIRFM technique. Perhaps a special laser setup such that multiple fluorescence dyes are being excited and monitored at the same time could capture the real-time localized reaction. Recently, multiple alternative super-resolution techniques such as PAINT ${ }^{136}$, DNA-PAINT ${ }^{137}$, and qPAINT ${ }^{138}$ could be explored to measure the localized reaction rate. 

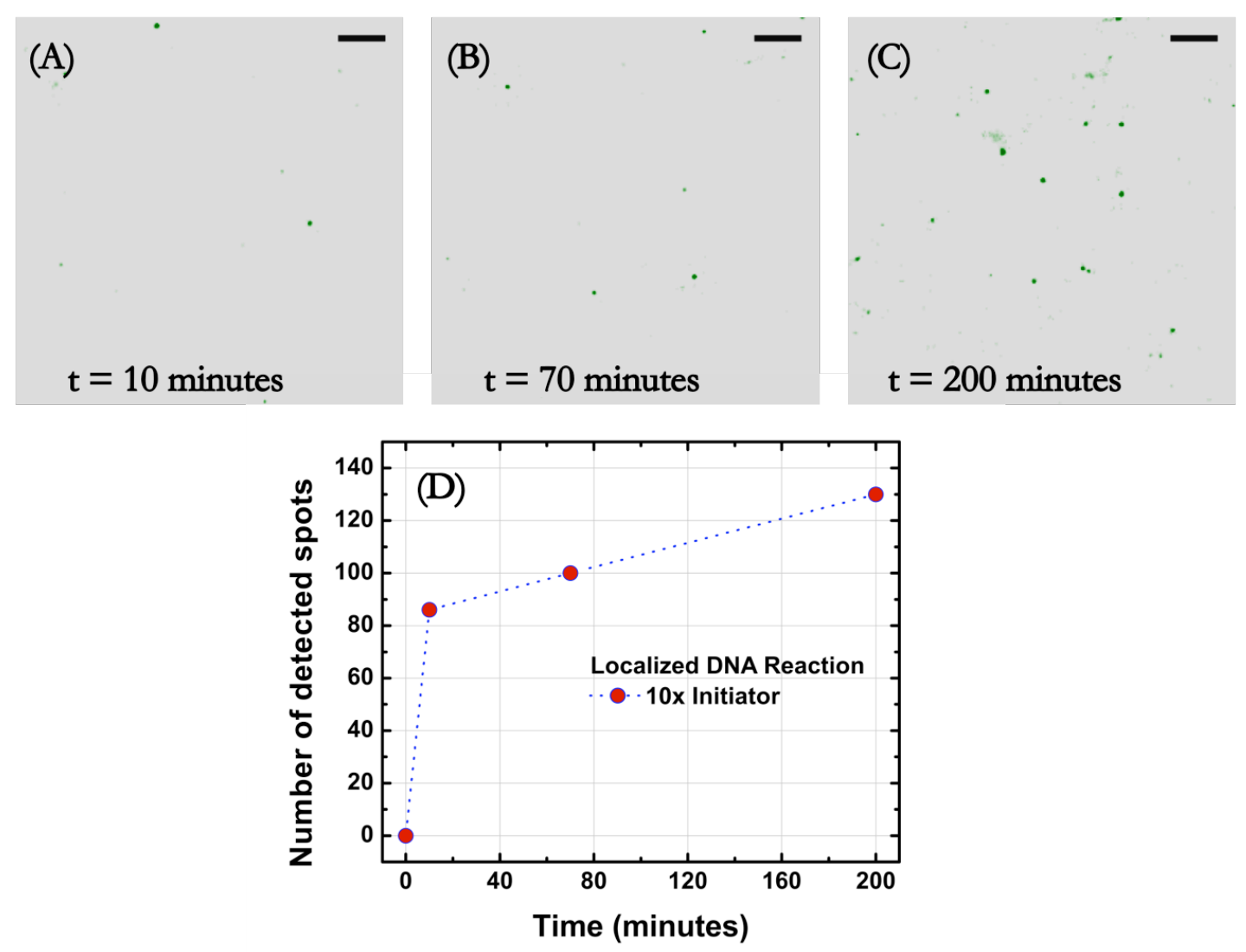

Figure 4.7: Visualizing localized DNA hybridization chain reaction via TIRF. Fluorescence emission of detecting the reaction completion overtime (10 (A), 70 (B), and 200 (C) minutes). Number of detected spots increased as thermal equilibrium was reached (D). All scale bars are $1 \mu \mathrm{m}$ and these images are superresolved with uncertainty between 10 and $20 \mathrm{~nm}$. Refer to Figure 4.8 for additional data.

To quantify the yield of the localized DNA hybridization reaction on DNA origami rectangle, TIRFM data were analyzed as shown in Figure 4.7. In particular, the binding event of the dye-labeled output strand was monitored while exciting the dye molecule at the same time. Only when the strand became bound to the last hairpin was the emission detected due to the TIRF effect. This 
binding event also indicates that the localized DNA hybridization reaction has reached completion. At the beginning ( 10 minutes), the number of emission events slowly increased (Figure 4.7A). The number of emission events continued rapidly increasing until thermal equilibrium was reached (Figure 4.7B-C). The number of emission events was plotted over time as shown in Figure 4.7D. These results indicate that the localized DNA hybridization reaction occurred on the surface of the DNA origami rectangle. The maximum number of emission spots was observed at thermal equilibrium. It is worth noting that the localized reaction rate is expected to occur fast; however, the increasing number of emission events over 200 minutes indicates that the initiation and termination processes could be experiencing the rate-limiting step due to the diffusion of the initiator and the output sequences. 


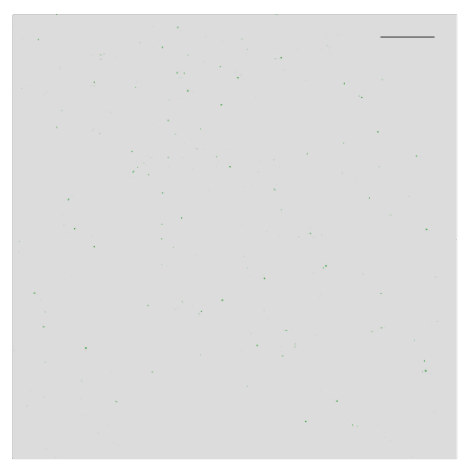

(A)

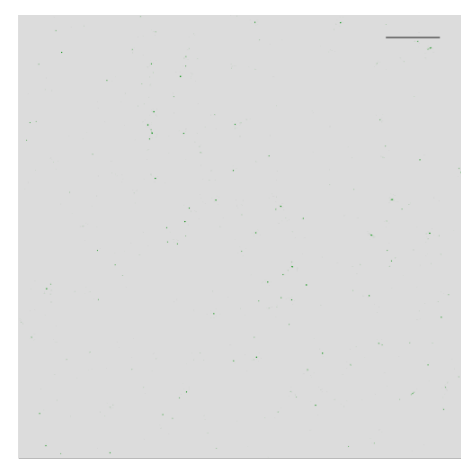

(B)

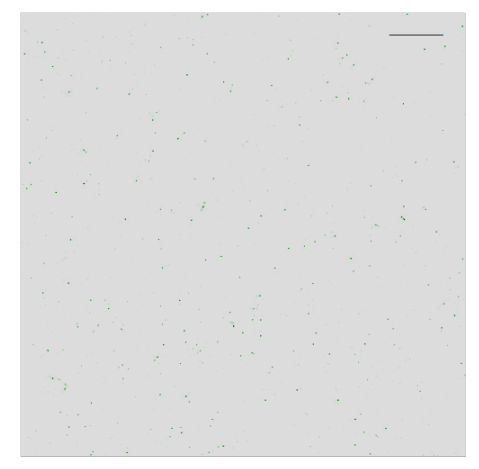

(C)

Figure 4.8: Super-resolved data after exciting the dye-labeled output strand while monitoring the emission overtime: (A) 10 minutes, (B) 70 minutes, and (C) 200 minutes. Scale bars are $5 \mu \mathrm{m}$.

\subsection{Ensemble Fluorescence Characterization}

Ensemble fluorescence spectroscopy was also performed to observe the

kinetics of localized DNA hybridization reaction. A fluorescence reporter complex pair was utilized to observe changes in the kinetics of a localized cascade DNA hybridization chain reaction. In particular, the reporter complex binds to the last hairpin when it is opened, signifying the chain reaction is complete (illustrated in Figure 4.9A). Upon adding the initiator to the solution, the fluorescence emission rapidly increased and saturated after 200 minutes as shown in Figure 4.9B (green curve). A control experiment was performed in the absence of the origami rectangle. Upon adding the initiator to the solution, the fluorescence emission increases slowly as shown in Figure 4.9B (purple curve).

The ensemble fluorescence response was significantly stronger for the cascade 
reaction in the presence of the origami rectangle than in the absence of the origami rectangle. This indicates that constraining DNA hairpins onto DNA origami rectangles of the same cascade reaction enhanced the reaction rate. The localized cascade reaction reached 50\% completion within 200 minutes whereas the cascade reaction in the absence of DNA origami rectangle slowly increased even after 800 minutes. The ensemble fluorescence data at 50\% completion of the proposed system was strongly correlated with the maximal number of emission events which were observed in TIRFM data in Figure 4.7D.

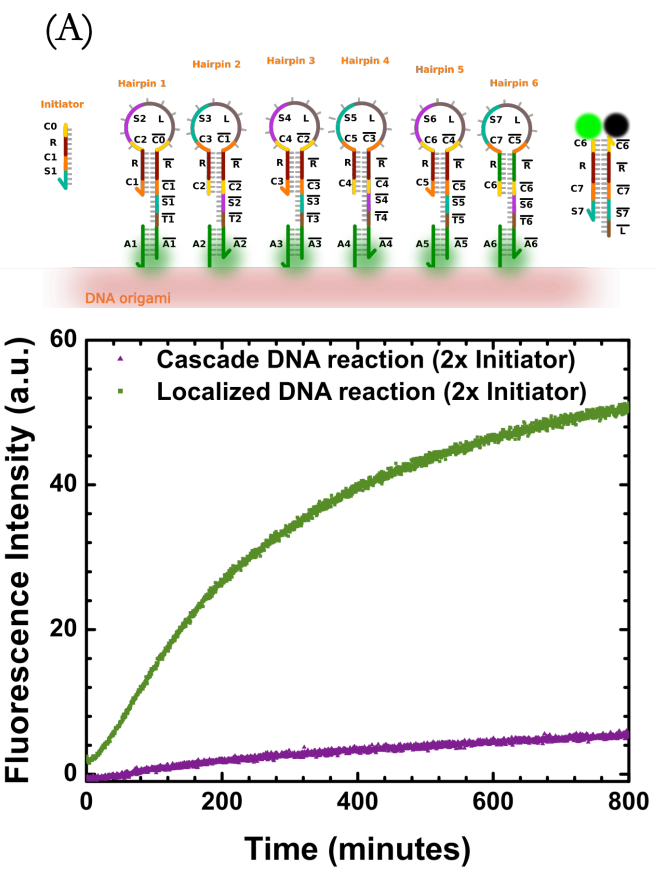

(B)
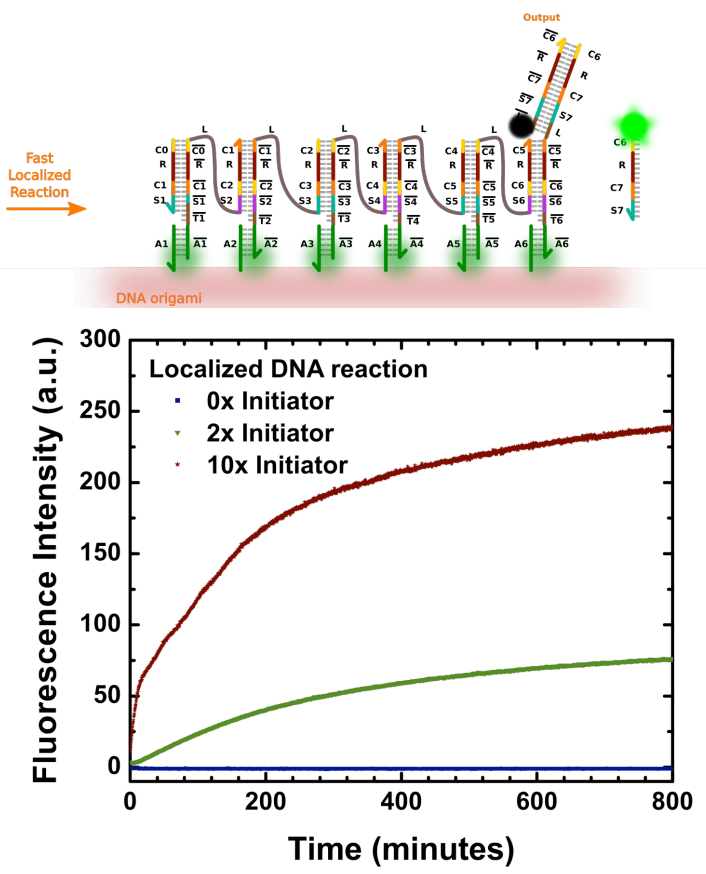

(C)

Figure 4.9: Ensemble fluorescence characterization. (A) Schematic of localized cascade DNA hybridization chain reaction on the surface of DNA origami rectangle before and after the completion. (B) Kinetic characterization of 
localized cascade DNA hybridization chain reaction on DNA origami rectangle (green curve). Kinetic characterization of cascade DNA hybridization chain reaction without DNA origami rectangle (purple curve). Each solution was kept at $5 \mathrm{nM}$ concentration and the initiator was added in $2 x$ excess. (C) Effect of localized cascade DNA hybridization chain reaction rate as a function of initiator concentration.

Since the initiator was not tethered to DNA origami, displacing the first hairpin could be the rate-limiting process. To observe the effect of localized cascade DNA hybridization reaction rate as a function of initiator concentration, various concentrations of the initiator was tested as shown in Figure 4.9C. At 0x initiator, the fluorescence emission was minimal, indicating that the localized cascade DNA hybridization chain reaction was not significant (blue curve). At 10x initiator, the fluorescence emission was rapidly increased compared to $2 \mathrm{x}$ initiator, indicating that the rate-limiting step is responsible for slowing down the speed of the localized reaction. To overcome the rate-limiting process of initiating the localized cascade DNA hybridization chain reaction, photoactivation mechanism utilizing photo-cleavable molecules could be explored as a replacement method. This result also indicates that displacing the reporter complex by the last hairpin could also be limited by the rate-limiting process. The reporter complex could be tethered to DNA origami platform to minimize the rate-limiting process. In fact, a fluorescent base (2-aminopurine) was 
modified to the last hairpin as an alternative approach to overcome the ratelimiting step; however, the fluorescence emission was significantly minuscule for concentration less than $1 \mu \mathrm{M}$ (data not shown).

\subsection{Conclusions}

Prior dynamic DNA nanodevices made use of DNA strands to encode values and used DNA hybridization reactions to perform computations. Most of these prior DNA computation systems relied on the diffusion of DNA strands to transport values during computations. During diffusion, DNA molecules randomly collide in solution. At low concentrations and temperatures, diffusion can be quite slow and could impede the kinetics of these systems. At higher concentrations and temperature, unintended spurious interactions during diffusion can hinder the computations. Hence, increasing the concentration of DNA strands to speed up DNA hybridization reactions has the unfortunate side effect of increasing leaks, which are undesired hybridization reactions in the absence of input strands. Also, diffusion-based systems possess global states encoded via concentration of various species and hence exhibit only limited parallel ability.

To address these challenges, this chapter made use of a novel design for DNA computation called a localized hybridization network, where diffusion of 
DNA strands does not occur. (In contrast, all prior attempts to do localized DNA computation have required some diffusion of DNA strands during intermediate stages of the computations.) Instead all the DNA strands are localized by attaching them to DNA origami which provided an addressable substrate for the strands.

The experiments demonstrated that DNA hairpins were successfully selfassembled to the surface of a DNA origami rectangle. In addition, the hairpins were successfully programmed to undergo cascade DNA hybridization chain reaction on the surface of DNA origami rectangle via AFM, TIRFM, and ensemble fluorescence spectroscopy.

It is also shown that the speed of DNA hybridization reactions by tethering DNA hairpin sequences to the surface of DNA origami rectangle has a significant effect over DNA hybridization reactions in the absence of a DNA origami surface. This indicates that localization increases the relative concentration of the reacting DNA strands, thereby speeding up the kinetics. This chapter demonstrated a localized hybridization network that executed a chain reaction of six DNA hybridizations which executes faster than nonlocalized DNA reactions. 
With the advantage of controlling the reaction rate on the surface, the proposed system may be used in the future to target surface-bound proteins and perform computation on cellular membranes. The potential of DNA nanotechnology towards biological applications may be soon realized in disease detection and prevention.

Table 4.1: DNA sequences for tethering DNA origami to the glass's surface via biotin-streptavidin conjugation.

\begin{tabular}{|l|l|}
\hline Unmodified Staple Strand & Modified Staple Strand \\
\hline SS20, ACG GCT ACA AGT ACA ACG & Biotin-TTTT-ACG GCT ACA AGT ACA ACG GAG ATT CGC \\
GAG ATT CGC GAC CT & GAC CT \\
\hline SS88, AAG AGG AAC GAG CTT CAA & Biotin-TTTT-AAG AGG AAC GAG CTT CAA AGC GAA AGT \\
AGC GAA AGT TTC AT & TTC AT \\
\hline SS190, CTA AAA TAA GTA TTA ACA & Biotin-TTTT-CTA AAA TAA GTA TTA ACA CCG CCT CGA \\
CCG CCT CGA ACT GA & ACT GA \\
\hline SS222, AGA TTA GAT TTT CCA GCA & AGA TTA GAT TTT CCA GCA GAA GAT AAA AAA TAC \\
GAA GAT AAA AAA TAC CGA & CGA - TTTT-TAGGTAAGAATGATGTATGTGTGG (marker) \\
\hline
\end{tabular}

Table 4.2: DNA oligomers for constructing the proposed system. For kinetic studies, RC duplex is displayed by hairpin $\mathrm{H6}$.

\begin{tabular}{|c|c|c|}
\hline Name & Sequence $\left(5^{\prime}\right.$ to $\left.3^{\prime}\right)$ & Length (nt) \\
\hline Hairpin H1 & $\begin{array}{l}5^{\prime} \text { linker- } \\
\text { GGGTGGGTTGTGATGTATGTAATGTTTTCCTCCTACTCACATAC } \\
\text { ATCACAAC-3' }\end{array}$ & 52 \\
\hline Hairpin $\mathrm{H} 2$ & $\begin{array}{l}5^{\prime}- \\
\text { ACTCACATACATCAATCGTCTTTCTTTTGTTGTGATGTATGTGA } \\
\text { GTAGGAGG }-3^{\prime} \text { linker }\end{array}$ & 52 \\
\hline Hairpin H3 & $\begin{array}{l}5^{\prime} \text { linker - } \\
\text { GAAAGACGATTGATGTATGTGAGTTTTTTCTTCCTTTAACATAC } \\
\text { ATCAATCG - 3' }\end{array}$ & 52 \\
\hline Hairpin $\mathrm{H} 4$ & $\begin{array}{l}5^{\prime}- \\
\text { TTTAACATACATCACCACTTTCCTTTTTCGATTGATGTATGTTA } \\
\text { AAGGAAGA - 3' linker }\end{array}$ & 52 \\
\hline Hairpin H5 & $\begin{array}{l}5^{\prime} \text { linker - } \\
\text { AGGAAAGTGGTGATGTATGTTAAATTTTCTTCTCCTCCACATA } \\
\text { CATCACCAC - 3' }\end{array}$ & 52 \\
\hline Hairpin H6 & $\begin{array}{l}\text { 5'- } \\
\text { CTCCACATACATCATATTCCCTCATTCAAGCGTGGTGATGTAT }\end{array}$ & 55 \\
\hline
\end{tabular}




\begin{tabular}{|c|c|c|}
\hline & GTGGAGGAGAAG - 3' linker & \\
\hline RC FQ & 5' - TTGAATGAGGGAATATGATGTATGTGG - 3' /Quencher/ & 27 \\
\hline RC TET & /Fluorophore/ 5' - CCACATACATCATATTCCCT - 3' & 20 \\
\hline RC Signal & $5^{\prime}$ - CCACATACATCATATTCCCTCATTCAA - 3' & 27 \\
\hline Initiator I1 & $5^{\prime}$ - CATTACATACATCACAACCCACCC - 3' & 24 \\
\hline Anchor A1 & $5^{\prime}$ - TTGAGAGTAGTGGAGGAGAGGAGA - H1 & 24 \\
\hline Anchor A2 & H2 - AGAAGGAAGGAAAGGAAAGAAGGA - 3' & 24 \\
\hline Anchor A3 & $5^{\prime}$ - TTGGTGTGGTGTGGTGTGGTGGTG - H3 & 24 \\
\hline Anchor A4 & H4 - GGAGGAGGAGAAGGAGAGGAGAGA - 3' & 24 \\
\hline Anchor A5 & 5' - GAAGTGAAGAAGAAGAAAGAGAAG - H5 & 24 \\
\hline Anchor A6 & H6 - GAAGAAAGAAGAAGAAGAGAGAAA - 3' & 24 \\
\hline Staple L1 & 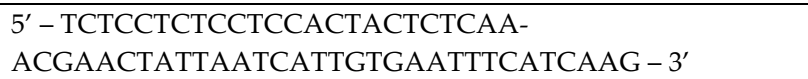 & 56 \\
\hline Staple L2 & $\begin{array}{l}5^{\prime} \text { - TTTCAACTACGGAACAACATTATTAACACTAT- } \\
\text { TCCTTCTTTCCTTTCCTTCCTTCT - 3' }\end{array}$ & 56 \\
\hline Staple L3 & $\begin{array}{l}5^{\prime}-\text { CACCACCACACCACACCACACCAA- } \\
\text { ACTGGATATCGTTTACCAGACGACTTAATAAA - 3' }\end{array}$ & 56 \\
\hline Staple L4 & $\begin{array}{l}\text { 5' - CATAACCCGCGTCCAATACTGCGGTATTATAG- } \\
\text { TCTСТССТСТССТTСТССТССТСС - } 3^{\prime}\end{array}$ & 56 \\
\hline Staple L5 & $\begin{array}{l}\text { 5' - CTTCTCTTTCTTCTTCTTCACTTC- } \\
\text { GAAGCAAAAAAGCGGATTGCATCAATGTTTAG - 3' }\end{array}$ & 56 \\
\hline Staple L6 & $\begin{array}{l}5^{\prime}-\text { TCAGAAGCCTCCAACAGGTCAGGATTTAAATA- } \\
\text { TTTCTCTCTTCTTCTTCTTTCTTC - 3' }\end{array}$ & 56 \\
\hline I1-TAM & 5' - CATTACATACATCACAACCCACCC - 3'/ TAM/ & 24 \\
\hline O6-FAM & /FAM/ 5' - TTGAATGAGGGAATATGATGTATGTGG - 3' & 27 \\
\hline M-ATTO & 5' - CCACACATACATCATTCTTACCTA - 3' /ATTO647/ & 24 \\
\hline
\end{tabular}




\section{Localized DNA Computation for Constructing Molecular-Scale Synthetic Neurons}

It's been demonstrated experimentally in the previous chapters that localized DNA hybridization reactions are feasible on DNA nanostructures and the reactions occur faster than non-localized DNA hybridization reactions. The implication of creating molecular synthetic circuits is now possible. In particular, bridging the solution-based and surface-based DNA devices to fully exploit biochemical applications is envisioned to take place in the near future. The next step is to demonstrate the following capabilities in order to fully construct and perform localized DNA computation: 1) Redundancy - DNA gates need to have a mechanism to compensate for errors (e.g. synthesis truncation or synthesis defects). This can be accomplished in the later section; 2) Reusability - DNA gates need to be constructed such that the reaction can proceed forward as well as backward. This can be accomplished by introducing novel concepts from prior studies such as toehold-exchange or catalytic circuits; 3) Resetability - DNA gates or DNA circuits need to have a mechanism to revert back to the previous state for the next computation to take place. This can be accomplished by introducing encoded dormant domains inside the circuits for later activation when the reset is needed to be done. 


\subsection{Motivation}

As DNA nanotechnology becomes more fully developed, localized DNA computation capable of carrying computational tasks will find their use as scientific instruments or as diagnostic tools. DNA nanostructures such as DNA origami provide impressive ability to rapidly design and self-assemble complex 3D structures at the molecular-scale. Dynamic DNA devices such as DNA walkers and other DNA robotic devices, provide locomotion capabilities over self-assembled DNA nanostructures. DNA computational devices such as DNA hairpins and DNA seesaw gates allow molecular-scale computations on the surface of self-assembled DNA nanostructures. This chapter attempts to demonstrate a novel combination of structural DNA nanotechnology (in particular DNA origami) and dynamic DNA nanotechnology (toehold mediated strand displacement). A novel class of DNA devices (DNA hairpins and DNA seesaw gates) tethered to a DNA origami surface is investigated. These devices can undergo local state transitions: transitioning between quiescent, excited, and refractory states similar to biological neurons. This molecular-scale excitable medium enables many exciting application opportunities in molecular sensing and control. 
The following section will outline prior research that are pertinent to the feasibility of constructing excitable medium for molecular synthetic neurons.

\subsubsection{Prior Work in DNA Structural Nanoscience: DNA Nanostructures}

Prior Work in DNA Tiles and Lattices: Self-assembly, programmability, and predictability are the major properties of DNA. Seeman et al. reported the first demonstration of creating lattices from a finite set of DNA sequences in early $1980 \mathrm{~s}^{14}$. Subsequently Reif et al. demonstrated a wide range of nanostructures such as TX tiles ${ }^{23}$, crossover tiles, 3D tiles, lattices ${ }^{139}$, and 3D lattices ${ }^{140}$. Currently many exquisite nanoscale structures and devices have been reported ${ }^{12,13,49,50,52,57,58,125,141}$ using DNA self-assembly.

Prior Work in DNA Origami: In contrast to the self-assembly of DNA lattices from DNA tiles, DNA origami is based on nucleated self-assembly and provides a systematic approach to design any arbitrarily nanoscale shapes and objects $^{15}$. In general, a long DNA scaffold is used as the nucleation template for short DNA sequences to form desired complex patterns. After 10 years, many exquisite nanoscale objects ${ }^{142}$ have been constructed using DNA origami method ranging from $2 \mathrm{D}$ rectangles to $3 \mathrm{D}$ mechanical gears. It's beneficial to utilize the 
large surface area of DNA origami nanostructures in order to arrange DNA components for constructing functional excitable DNA-based materials.

\subsubsection{Molecular Scale Imaging of DNA Nanostructures}

AFM imaging is a technique of scanning probe microscopy and has been used ubiquitously to observe structural topologies with sub-10 nm resolution. AFM imaging has been utilized to characterize DNA lattices, DNA origami nanostructures, and modified DNA nanostructures either in dry or liquid mode conditions. The attachment of DNA hairpins, streptavidin molecules, and other nanoparticles on DNA origami nanostructures has been demonstrated in multiple studies.

Super-Resolution Imaging of DNA Nanostructures: The minimum discernable

distance between objects is given by $d=\frac{\lambda}{2} \times N A$, where $N A$ is the numerical aperture of the lens and $\lambda$ is the wavelength of light. This is called the diffraction limit of light and it is usually $\sim 200 \mathrm{~nm}$. In the last two decades, several techniques have been developed to overcome this limit. The main objective in these techniques attempts to observe temporal separation of objects which cannot be spatially separated at optically-resolvable resolution by conventional microscopy. Post processing of temporal images enables the creation super- 
resolved images. In the past decade, a number of far-field (super-resolution) microscopy techniques, which can circumvent light's diffraction barrier ( 200 $\mathrm{nm})$, were proposed ${ }^{136,137,143-149}$. These include both 2-D and 3-D techniques with much higher lateral and axial resolution ${ }^{136,137,143-148}$. Recently, Jungmann et al. introduced the DNA-PAINT super-resolution imaging technique to the field of DNA nanoscience to observe the kinetics of DNA hybridization reaction ${ }^{137}$. In particular, fluorescent-labeled DNA strands in solution repeatedly hybridize and dehybridize to specific DNA strands on the surface of a DNA origami. DNAPAINT improves on previous techniques since it is not prone to photo-bleaching (a usual problem with most fluorescent imaging techniques). The multiplexed version of this technique is called exchange-PAINT and they used this to image numbers on a DNA origami rectangle ${ }^{150}$. In addition to AFM and fluorescencebased techniques, super-resolution imaging techniques such as DNA-PAINT will provide additional capability to probe dynamic DNA systems when constructing excitable medium.

DNA Computing and Catalytic Reactions: A catalytic reaction is defined as a regular DNA hybridization reaction with an addition of a catalyst molecule and 
has two distinct properties: (i) the speedup of the target reaction and (ii) the rerelease of the catalyst to allow for multiple turnover.

DNA Computing and DNA Robotics: DNA self-assembly has been used to perform computation and construct nanoscale robotics. There has been active investigation of DNA self-assembly for molecular computation as well as for creating novel nanodevices. Several novel DNA computing architectures ${ }^{151-155}$ such as molecular parallelism to perform cumulative XOR using triple-crossover molecules, pairwise XOR using DNA string tile self-assembly, autonomous DNA cellular automata, biomolecular devices executing process algebra were reported. In addition, several novel DNA-based robotic devices ${ }^{70,156-162}$ such as autonomous DNA nanomechanical devices with rolling and walking capabilities, unidirectional walkers with autonomous motions along a track, DNA nanoactuators, DNA nanotransport devices powered by polymerase $\varphi 29$, and nanorobtic devices using DNAzymes were achieved.

DNA Hybridization Reactions: Recently, researchers have been exploiting DNA hybridization for constructing nanostructures as well as programming molecular devices ${ }^{12,13,48-58}$. Many ingenious DNA devices based on DNA strand displacement have been experimentally demonstrated, including the 
construction of molecular systems ${ }^{27}$, logic circuits $^{4,59}$, sensors ${ }^{60}$, amplifiers ${ }^{61-63}$, molecular machines ${ }^{64-67}$, walkers ${ }^{63,64,68-95}$, robots ${ }^{69,79,80}$, chemical controllers ${ }^{96}$, and neural networks ${ }^{97}$ was realized. The kinetics of DNA hybridization reactions depends on diffusion of DNA molecules which randomly collide and interact in solution. To speed up DNA hybridization reactions, a high concentration of DNA molecules is often used; however, leaks (undesired hybridization reactions in the absence of input strands) frequently occur in DNA hybridization reactions at high concentrations. As a result, undesired DNA hybridization reactions halt designed DNA hybridization reactions from occurring.

Toehold-Mediated Strand Displacement: Many devices in dynamic DNA nanotechnology employ a technique termed toehold-mediated strand displacement, where a single stranded DNA binds to a single-stranded toehold region (known as the toehold) of a DNA complex, and then extends (via a branch migration process) it's initial hybridization at the toehold region to displace one of the strands bound to DNA complex. The overall effect is that one of the strands in the complex is replaced with another one. Toehold-mediated strand displacement can be used to allow a DNA structures to change its state in response to the addition of a new nucleic acid strand. Yurke et. al. first 
demonstrated toehold-mediated strand displacement in their nanomachine ${ }^{26}$. Many DNA hybridization reactions based on toehold-mediated strand displacement have been studied in the past decade and are widely used to operate nanoscale machines and construct DNA circuits $4,26,27,30,32,33,59,61,97,114-117$. Using toehold exchange strand displacement, Winfree's group demonstrated various DNA computation via seesaw gates ${ }^{4}$ with up to 130 DNA sequences.

DNA Hairpin Systems: An oligonucleotide with a secondary structure consisting of a duplex stem and an intermediate single-strand loop is called DNA hairpin. A DNA hairpin which is labeled with a fluorophore and quencher pair is called a molecular beacon and is used to probe changes in biological environment. DNA hairpin systems have been used catalytically and noncatalytically to drive DNA hybridization reactions including dynamic DNA nanomachines and DNA walkers ${ }^{63,64,68-95}$. Other researchers created DNA hairpin systems for constructing dynamic DNA nanomachines. Pierce et al. programmed a pair of DNA hairpins that formed a linear growth nanostructure. Pierce's group reported the first DNA hairpin catalytic reactions ${ }^{100}$, known as HRC, which made use of toehold-mediate strand displacement to trigger an isothermal DNA amplification for highly sensitive and selective fluorescent detection. The 
use of DNA hybridization as an energy source has been a main theme in many DNA systems. An alternative energy source could also come from metastable $\mathrm{DNA}^{98}$. For instance, Turberfield et. al. used fuel sourcing from DNA hairpin loops to operate DNA motors ${ }^{99}$. In addition, energy sources from DNA hairpins have been used to assist with catalytic hybridization ${ }^{31}$ and triggered selfassembly ${ }^{63,100}$.

Localized DNA Hairpin Systems: Solution based systems for DNA computation demonstrate the enormous potential of DNA nanosystems. But most of the systems rely on diffusion based hybridization to perform complex state changes/computation. At low concentrations and temperatures, diffusion can be quite slow and could impede the kinetics of these systems. At higher concentrations and temperature, unintended spurious interactions (often called leaks) could hinder the systems from its programmed trajectory. Localized hybridization networks are set of DNA strands attached to an addressable substrate. This localization increases the relative concentration of the reacting DNA strands thereby speeding up the kinetics. Diffusion based systems possess global states encoded via concentration of various species and hence exhibit limited parallel ability. In contrast, localized hybridization systems allow for 
each copy of the localized hybridization network to operate independently of each other. Localized hybridization networks also allow one to reuse the same DNA sequence to perform different actions at distinct locations on the addressable substrate, increasing the scalability of such systems by exploiting the limited sequence space. An advantage of localized hybridization computational circuit is sharper switching behavior as information is encoded over state of a single molecule. This also eliminates the need for thresholding, as computation is performed locally eliminating the need for a global consensus. These advantages are expounded in greater detail in Chandran et al. ${ }^{163}$. Quite recently, others ${ }^{33,164}$ have also demonstrated that locality can be used successfully. Recently, theoretical models to describe DNA hybridization reactions on nanoscale substrates have been proposed by others $5,104,113,118$. The theoretical models indicate strong evidence of speedup as well as leak reduction for those systems in which DNA gates are tethered to substrates so as to locate the DNA gates in proximity. In terms of experimental demonstration, Kopperger et al. studied the diffusion transport mechanism of linear DNA strands moving on the surface of a DNA origami rectangle via surface-bound sticky strands ${ }^{112}$. Teichmann et al. studied a similar effect of the diffusion transport mechanism of single-stranded DNA 
moving on the surface of DNA origami rectangles via surface-bound sticky strands as a function of spatial distance ${ }^{105}$. From understanding the spatial arrangement of DNA molecules on nanoscale surfaces, Ruiz et al. reported a simple surface-bound DNA amplification circuit using the concept of seesaw gates $^{111}$. A common strategy of the above studies is to bind DNA strands to DNA nanostructures to exploit the effects of localization. However, the strands are free to travel beyond the reaction surface during DNA hybridization events due to the strands being not physically attached to the surface. The probability of having DNA strands flow away from the surface is higher than when the DNA strands are physically tied down to the surface. Recently Yin et. al. employed triggered self-assembly hybridization chain reaction $(\mathrm{HCR})^{100-102,122,123}$ to program DNA that could form and grow nanoscale structures ranging from polymer-like oligomers to branched oligomers and even dendritic oligomers ${ }^{63,124}$. Chandran et al. reported a well-define architecture to construct localized hybridization circuits $^{104}$. In particular, several fundamental logical gates - AND, OR, propagation, and a degree two fan-out gates, were designed to work on addressable substrates. In addition, a biophysical model of substrate-tethered hybridization was proposed to describe the benefit of localization. Simulations of 
tethered hybridization circuits using visual DSD showed marked improvement in overall reaction rates. Encouraged by these results, a four bit square root circuit was successfully implemented ${ }^{163}$. Recently, Dalchau et al. reported a latest studies of localized hybridization circuits in $2015^{110}$. In addition to the earlier architecture, the latest system used continuous-time Markov chain (CTMC) to analyze the chemical master equation - a major component in solving DNA hybridization reactions. Due to a low copy number of molecules constrained by the spatial arrangement, the techniques for the analysis of Markov processes must be considered for model analysis to be relevant. It's well known that, as the copy number of a discrete stochastic system decreases, mass action ordinary differential equation (ODE) approximation breaks down and stochastic simulation methods should be used. A stochastic system involves the analysis of all possible interactions of the system to determine the probability of a specific behavior. This technique considers all possible states of a closed system and the rates of transition between each state to form a CTMC. The distribution of all possible interactions produced by the stochastic simulation algorithm (SSA) to perform numerical integration of the chemical master equation (CME). The full state space of the system is encapsulated within the continuous-time Markov 
chain and is used to analyze the chemical master equation. The probabilistic analysis of localized DNA hybridization circuits effectively measured the behaviors of localized circuits for a broad range of localized concentrations and has been showed to improve the correctness of our localized systems reported earlier ${ }^{110,163}$. Recently a number of localized hybridization schemes have been implemented experimentally ${ }^{6,165,166}$. In particular, two separated systems have been implemented in which hairpin strands are tethered to the surface of DNA nanotrack and DNA origami rectangle, separately ${ }^{6,166}$. DNA nanotrack provided a finite space to arrange up to 6 distinct DNA hairpins. The localized hybridization reaction rate has been showed to occur faster than non-localized hybridization reaction rate of the same system but without the presence of the nanotrack. Due to the finite space from the DNA nanotrack, DNA origami rectangle has been used instead. Another benefit of using DNA origami is the potential to attach at least 200 distinct DNA devices. Once attached on DNA origami rectangles, DNA hairpins have been showed to undergo localized DNA hybridization reactions. The localized reaction rate has been determined to be faster than in the absence of DNA origami rectangle ${ }^{166}$. 


\subsubsection{Prior Work in Excitable Medium and Synthetic Neurons}

An excitable medium ${ }^{167-169}$ is a system distributed in space with nonlinear dynamics, where at each location the medium can be in one of three states: (i) quiescent, where it is unexcited but has the capacity to be excited by immediate neighbors, (ii) excited, and (iii) refractory, where it has recently been excited but is not excitable for a period of time (the refractory period). Examples of excitable media in cellular biology include: neuron and cardiac muscle's atrial fibrillation ${ }^{170,171}$. Typically the local excitable medium responds very strongly to a relatively weak external stimulus, and the excitation obeys an all-or-nothing principle: the event of excitation does not depend much on the neighbor's excitation strength, as long as the level of the neighbor's total excitation exceeds some threshold. For example for neurons and cardiac muscle's atrial fibrillation, excitability from a quiescent state is elicited by a short, depolarizing electrical perturbation of the quiescent state trans-membrane potential, which as long as it is above a certain threshold, causes a spike of trans-membrane potential (known as an action potential). Other examples of excitable media include spatial propagation of diseases and forest fires. An excitable medium generally can propagate a single traveling wave of excitation as well as a series of traveling wave trains of excitation, each separated by refractory periods. There are various 
theoretical models of excitable media, most notably the Greenberg-Hastings cellular automaton ${ }^{172}$ (a cellular automaton) is an array of finite state automata whose state transitions depend on their own state and the state of their nearby neighbors ${ }^{171,173,174}$. The primary goal is to produce molecular-scale synthetic neurons, which have a dynamic behavior of excitable medium. Excitable media provides a basic way to attempt to perform computing based on the way the brain's neurons perform computations.

Excitable media can be configured as synthetic neurons. This allows one to attempt to perform computing based on the way the brain performs computations. In order to understand the fundamental operations and behaviors of neuron networks, different approaches have been undertaken such as synthesizing proteins to protect neurons and attempt to elucidate neuron behaviors, or developing synthetic brain imaging technique to observe brain activities under various conditions.

Prior Theoretical Models of Neurons: There are many theoretical models of biological neurons. An artificial neuron is a relatively simple class of mathematical models of a neurons that is employed in the definition of artificial neural networks; examples of artificial neurons include McCulloch- 
Pitts (MCP) neurons ${ }^{175}$ and other linear threshold functions ${ }^{176,177}$. Other more detailed models of neurons include Hodgkin-Huxley, Galves-Löcherbach, FitzHugh-Nagumo, Morris-Lecar, Hindmarsh-Rose, and various Markovian and non-Markovian models ${ }^{178}$.

Prior Carbon Nanotube Implementations of Neurons: Prior work has proposed the use of carbon nanotubes to preform excitatory and inhibitory dendritic computations ${ }^{179-182}$. However, carbon nanotubes are difficult to assemble in complex assemblies compared to DNA-nanostructure approaches.

Prior Analog Electronic Implementations of Neurons: There are a large number of papers on silicon-based synthetic neuron systems. Carver Mead ${ }^{183}$ pioneered the field of Neuromorphic computing and engineering, where integrated analog electronic systems are used to mimic neuro-biological architectures present in the nervous systems ${ }^{184}$. These have been used for many applications including perception and motor control.

Prior work on DNA-based Synthetic Neural Networks: A few attempt has been demonstrated to program DNA hybridization systems to mimic neural networks. For instance, Qian et. al. constructed DNA circuits that exhibit autonomous 
brain-like behaviors ${ }^{97}$ from a simple see-saw gate architecture to small artificial neural networks.

Prior Work in DNA Devices on Cells: Recently, intricate nanomachines have been demonstrated to deliver drugs to cancer cells ${ }^{185,186}$. Artificial DNA membrane channels have been shown to be able to transport ionic molecules across the cellular membranes ${ }^{187}$.

\subsection{Architecture to Construct Molecular-Scale Neurons}

To develop and experimentally test a type of excitable media that can be viewed as molecular-scale synthetic neurons, the following strategies are investigated. The excitable medium consists of DNA hairpins that are tethered and self-assembled to 2D DNA origami nanostructures. Their tethering position on the 2D origami will form dendritic trees similar to branched neurons. These DNA hairpins open up when entering their excited state, but are designed to eventually re-configure back to hairpins after a tunable time duration. When a DNA hairpin loop opens up, it exposes single-stranded sequences that are potentially capable of further activating (opening up) multiple other nearby DNA hairpins. This can be used to create a chain reaction of activations similar to the activation of the branches of a neuron. From prior work on localized DNA hairpin systems ${ }^{6,166}$, it's important to allow for a number of additional 
capabilities that include: (1) tethering of the DNA hairpins into complex patterns on DNA origami including dendritic trees and circular structures, (2) branching of the reaction chains by use of multiple domains of the opened DNA hairpins, and (3) autonomous resetting of the DNA hairpins to allow their re-use.

The following section will outline demonstrations of application prototypes of excitable medium including (a) molecular detection (sensing molecules) using the trees of hairpins, (b) performing branched tree computation on DNA origami, and (c) cyclic activation behavior using cycles of hairpins. In particular, various alternative uses of dendritic tree attachment patterning of the hairpins: (i) Logic OR of Multiple Possible Activations: The tree's leafs are activated by detection of molecules such as a DNA strand or other molecules (via an aptamer), and then the reaction sequence moves up the tree (with OR logical gates) to the root, which provides a photonic output indicating that at least one of the leafs of the tree detected an input molecule, and alternatively (ii) Multiple Replication of a Singe Activation: The root of the tree is activated by a molecular detection event, and the activation reaction proceeds down the tree to the leaves, all of which may for example provide photonics output. The first step is to fully develop different complex patterns on DNA 
origami nanostructures and provide the upper bound on the number of different complex patterns that can fit on DNA origami's surface given the size and scale of DNA scaffolds. The second step is to develop various error-correction schema to test branching of the localized chain reactions on the surface of DNA origami nanostructures. The third step is to develop autonomous resetting DNA hairpins to allow their re-use for later chain reactions. The fourth step is to create a number of prototype simulations and experimental demonstrations that combine complex patterns, branching and resetting capabilities to fully operate the excitable medium and demonstrate various functions mentioned previously.

\subsubsection{Error Correction Strategies}

Using Redundancy (via Branching) to Increase Chain Reaction Success: To develop various error-correction schema to test branching of the localized chain reactions on the surface of DNA origami nanostructures, each gate is encoded with multiple identical outputs and is tethered to a fixed substrate as shown in Figure 5.1. The operation of branching of the localized DNA hairpin systems is initialized by an initiator DNA sequence - the initiator sequence in the later design will be attached to the same substrate to fully explore the speedup factor due to the localized reaction. As a result, the activated gate $\left(G_{i}\right)$ will produce multiple identical outputs and these outputs can interact with the next hairpin 
gate $\left(\mathrm{G}_{\mathrm{i}+1}\right)$ as illustrated in Figure 5.1. To experimentally test branching capabilities, DNA origami rectangle will be employed. In particular, staple strands will be modified with sticky ends which will be used to assembly DNA gates. The branching increases (via redundancy) the likelihood of the reaction proceeding down the entire chain of hairpins via localized DNA hybridization reactions. Double (or multiple) chains of the same hairpin gates can be incorporated in order to increase the likelihood of the reaction by mitigating improper binding or missed intermediate DNA hairpin gates.

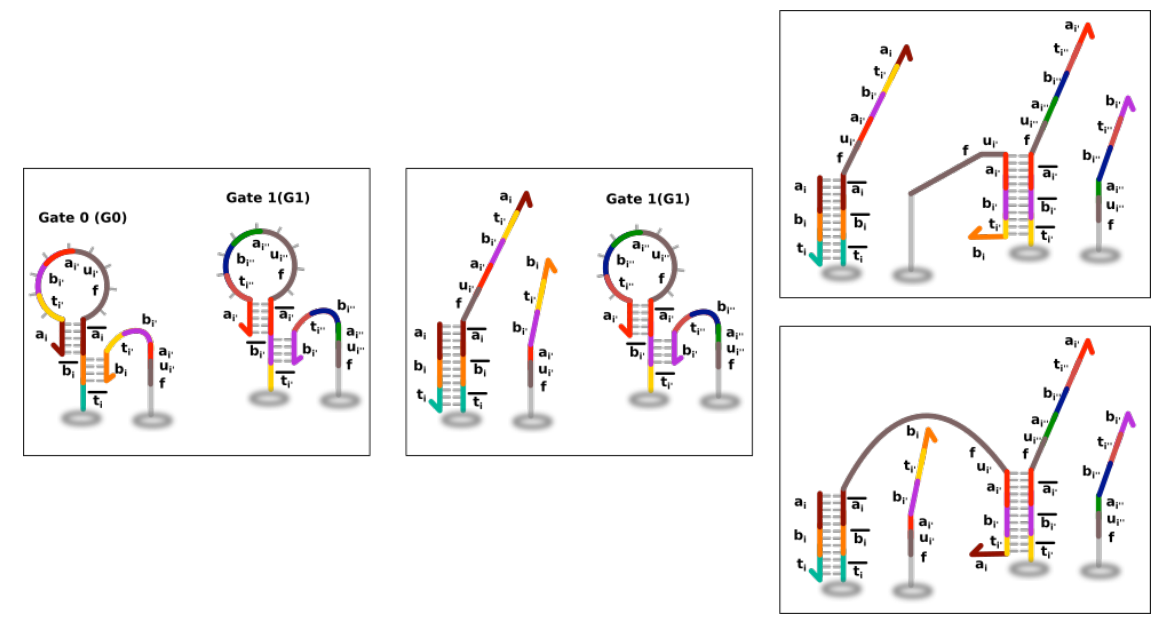

Figure 5.1: Using redundancy (via branching) to increase chain reaction success. Each gate is encoded with 2 identical outputs. After initiation, the gate Gi produces two identical outputs. Either of the outputs can trigger the next gate $\mathrm{Gi}+1$.

\subsubsection{Reversible Strategies}

To develop autonomous resetting DNA hairpins which allow for their reuse for later chain reactions, reversibility is achieved via using the toehold- 
mediated strand displacement. Each gate is encoded with an additional internal toehold domain $\left(\mathrm{u}_{\mathrm{i}}\right)$ and is tethered to a fixed substrate as shown in Figure 5.2.

The strategy to achieve the forward reaction is the following: the initiator triggers the forward reaction by binding to hairpin $\mathrm{H}_{\mathrm{i}}$ via the toehold domain $\mathrm{t}_{\mathrm{i}}$. The initiator then hybridizes to the stem domain ai via the branch migration mechanism. Since the other stem domain $b_{i}$ is designed to be short ( $\left.3 \mathrm{nt}\right)$, the melting temperature of the domain is around the operating temperature (i.e. $25^{\circ} \mathrm{C}$ ). Thus the entire stem of $\mathrm{H}_{i}$ eventually is being displaced and now can hybridize to the adjacent hairpin $\mathrm{H}_{\mathrm{i}+1}$.
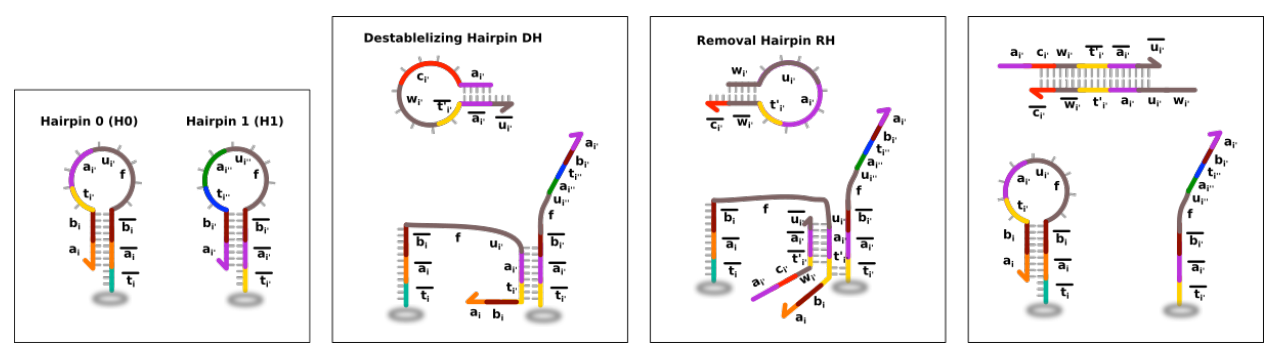

Figure 5.2: Reversible using toehold-mediate DNA hairpins. By controlling the DNA hybridization base pairing, the system is designed to revert back to the original state.

The strategy to achieve the reverse reaction is the following: the destabilizing hairpin $\left(\mathrm{DH}_{\mathrm{i}}\right)$ hybridizes to the toehold domain $\mathrm{u}_{\mathrm{i}}$ of the duplex formed by hairpin $\mathrm{H}_{\mathrm{i}}$ and $\mathrm{H}_{\mathrm{i}+1}$ and undergoes the branch migration to the remaining domains of $\mathrm{H}_{i}$ except the last three base pairs of $t_{i}$ domain. This 
reduces the stability of the prior duplex and delaminates $\mathrm{H}_{\mathrm{i}+1}$ from $\mathrm{H}_{\mathrm{i}}$. By introducing the removal hairpin $\left(\mathrm{RH}_{\mathrm{i}}\right), \mathrm{H}_{\mathrm{i}}$ can now fold back to the original state and is ready for the next imitation. Given the mechanism for the construction of a basic reversible system, the next step is to construct the systems with both errorcorrection scheme and reversible scheme. As illustrated in Figure 5.3, repeated attachment of hairpins can result in a branching system (e.g. error correction) or a tree-like system (e.g. binary tree) with controllable length.

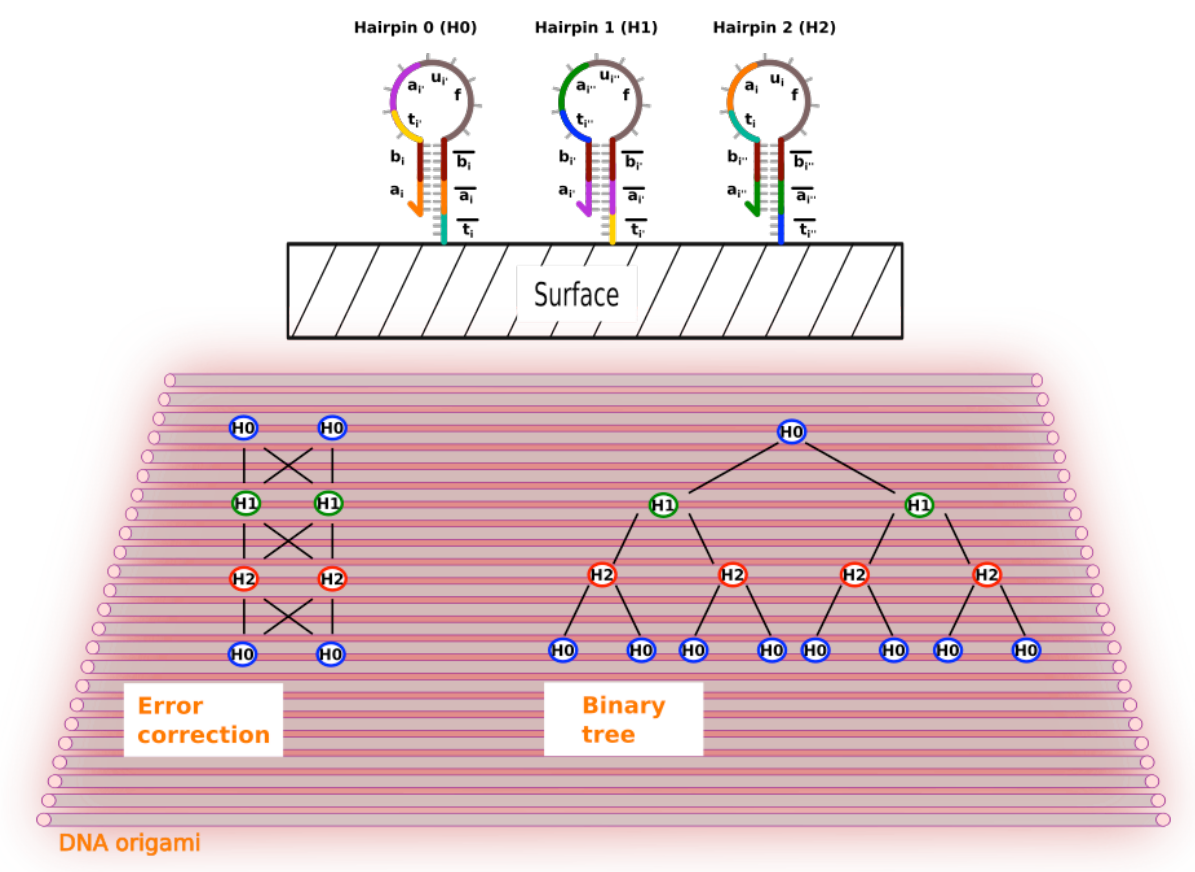

Figure 5.3: An architecture to construct error correction and branching tree. Using redundancy and branching to increase chain reaction success and to reset chain reaction. After initiation, the gate $\left(\mathrm{G}_{\mathrm{i}}\right)$ can hybridize to either the adjacent downstream gates $\left(\mathrm{G}_{\mathrm{i}+1}\right)$. After forward reactions, a set of destabilizing gates is introduced to delaminate the gates from the prior reactions. A complement set of removal hairpins is introduced to pull back DNA strands and allows gates to 
reconfigure back to the initial state. A detail of designing DNA sequences for the above strategies is presented in Appendix C.

\subsubsection{Dendritic Trees}

To construct dendritic trees, the systems need to have the errorcorrection/redundancy capability (to increase the propensity of the chain reaction) and to have the reversible capability (to reset the chain reaction). As illustrated in the previous section, the error-correction can be implemented by encoding multiple outputs in the same gate or by constructing multiple identical gates along the direction of the reaction (Figure 5.4A). To encode multiple outputs in the same gate, each gate is encoded with $n$ identical outputs and 1 internal toehold domain. The operation of branching of the localized DNA hairpin systems is initialized by an initiator DNA sequence. As a result, the activated gate $\left(\mathrm{G}_{\mathrm{i}}\right)$ will produce $n$ identical outputs and these outputs can interact with the next hairpin gate $\left(\mathrm{G}_{\mathrm{i}+1}\right)$ as illustrated in Figure 5.1. The reset operation of DNA hairpins for their re-use is similar to the strategy as illustrated in Figure 5.2. A set of destabilizing DNA sequences $\left(\mathrm{DH}_{\mathrm{i}}\right)$ is introduced and delaminates hairpin gates $\left(\mathrm{G}_{\mathrm{i}}\right)$ from the prior forward reactions. A complementary set of removal DNA sequences $\left(\mathrm{RH}_{\mathrm{i}}\right)$ is subsequently introduced to pull back DNA strands and away from hairpin gates and enables hairpin gates to reconfigure back to the initial state. 
The above proposed redundancy and resetting strategies as well as the large surface area of DNA origami rectangle makes it possible for building a dendritic binary tree. In particular, the input triggers the root node and the signal propagates down to the children nodes as illustrated in Figure 5.4B. In addition, two dendritic trees $\mathrm{T} 1$ and $\mathrm{T} 2$ with the common root allow for multiple inputs (tree T1) and multiple outputs (tree T2) ) to fan-in and fan-out the signal as shown in Figure 5.4C. The thermal equilibrium of these proposed dendritic trees can be validated using scanning probe microscopy and super-resolution fluorescence imaging. In particular, a subset of DNA origami staple strands is modified with linker strands that allow a subset of DNA hairpins to form a treelike structure on the surface of DNA origami rectangle. Since a tree-like structure is a collective of DNA hairpins, it is detectable via scanning probe microscopy. The amplified output can be observed using DNA-PAINT method. 


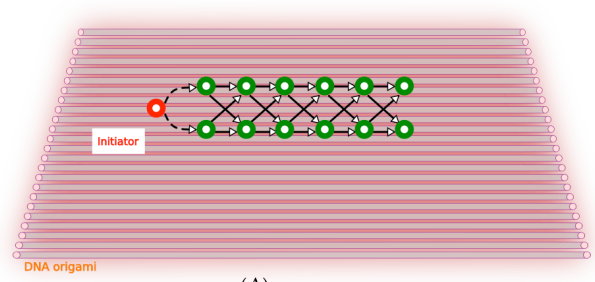

(A)

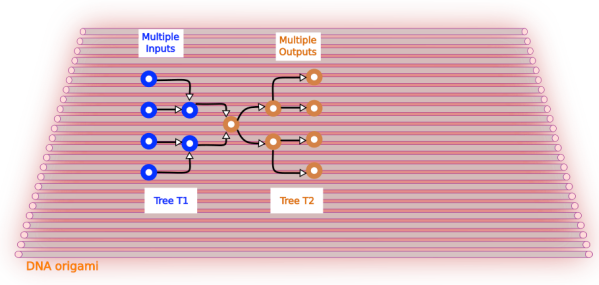

(C)

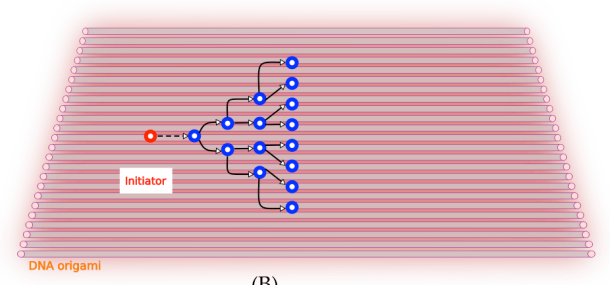

(B)

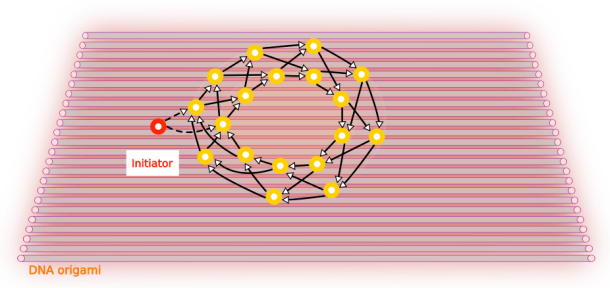

(D)

Figure 5.4: Using redundancy and resetting gates to construct dendritic trees. Actual implemented gates are indicated in dashed rectangle boxes. (A) A single branch of two linear chains, (B) A dendritic binary tree, and (C) Two dendritic trees T1 and T2 with the common root allowing for multiple inputs and multiple outputs, and (D) The cyclic patterning of activation signals is initiated once and is governed by the localized reactions. Note: each node is represented by a desired gate.

Using the above proposed redundancy and resetting gates, a scheme for cyclic network of hairpins attached to 2D origami is shown in Figure 5.4D. Each cycle gate is programmed to initiate the adjacent gate. After the initiation, the cyclic patterning of activation signals is governed by the localized reactions. This system is an extension from the system in Figure 5.4A with the last gate initiating the first gate. Experiments of a cycle and double cycle of DNA hairpins on DNA origami consist of a set of DNA origami staple strands that are modified with linkers which allow a set of DNA hairpins to bind and form a cycle along the circumference of DNA origami. The formation of cycle networks can be 
characterized using AFM. The perpetual motion of the cycle network can be observed using DNA-PAINT method. For this particular system, the notion of building an DNA-based oscillator or clock can be possible. The speed of the clock is controlled by the hybridization rate of the initiation step.

A key application of the dendritic trees to be experimental demonstrated is DNA detection amplification, where we will identify the presence of a small amount of target DNA sequence. Since the localized DNA hybridization reaction is highly specific and independent of diffusive DNA components, the event of detection of target DNA molecule is quickly amplified along the dendritic tree and results in a fluorophore blinking under super-resolution fluorescence imaging. Using dendritic trees, multiple outputs can be encoded to amplify the presence of target molecules. Previous studies indicate that DNA origami can sustain several hours in vivo, the proposed dendritic trees on DNA origami rectangle can also be used to monitor the presence small target molecules such as RNA $^{131}$, aptamers ${ }^{132}$, vaccines ${ }^{133}$ and drugs ${ }^{134}$. The use of cyclic networks as illustrated in Figure 5.4D of hairpin activation results in cyclic periods of activation of tunable duration. The resulting cyclic activation sequences may 
have important applications in control on molecular robotic devices, for example for control of a robotic device that repeatedly executes a sequence of movements.

\subsection{Summary}

In addition to creating molecular-scale synthetic neurons, there are many applications for localized hybridization networks. These include counting the number of disease marker molecules in a patient, detecting various cancer DNA sequences, and detecting and distinguishing bacteria by their distinguishing DNA. Recent advances in vitro evolution enabled the capability to generate a set of DNA aptamers that have a strong binding affinity to surface-bound proteins. The results from localized DNA hybridization networks may also be of practical use in performing surface computation on cellular membranes for disease detection and prevention. 


\section{Appendix A - Scripts and DNA Sequences}

The following scripts are used in Chapter 2 to design linear cascade DNA hybridization reactions. In particular, Script A.1 presents the NUPACK code to program DNA hairpins for constructing linear cascade DNA hybridization chain reactions. Script 2 presents the Matlab code to solve linear cascade DNA hybridization chain reactions. Script A.3 presents the Matlab code to solve for the rate constant using the least-squares method. The following tables shows DNA sequences for constructing the proposed DNA systems in Chapters 2, 3, and 4 . 


\section{Script A.1: NUPACK codes to program DNA hairpins for constructing linear cascade DNA hybridization chain reaction.}

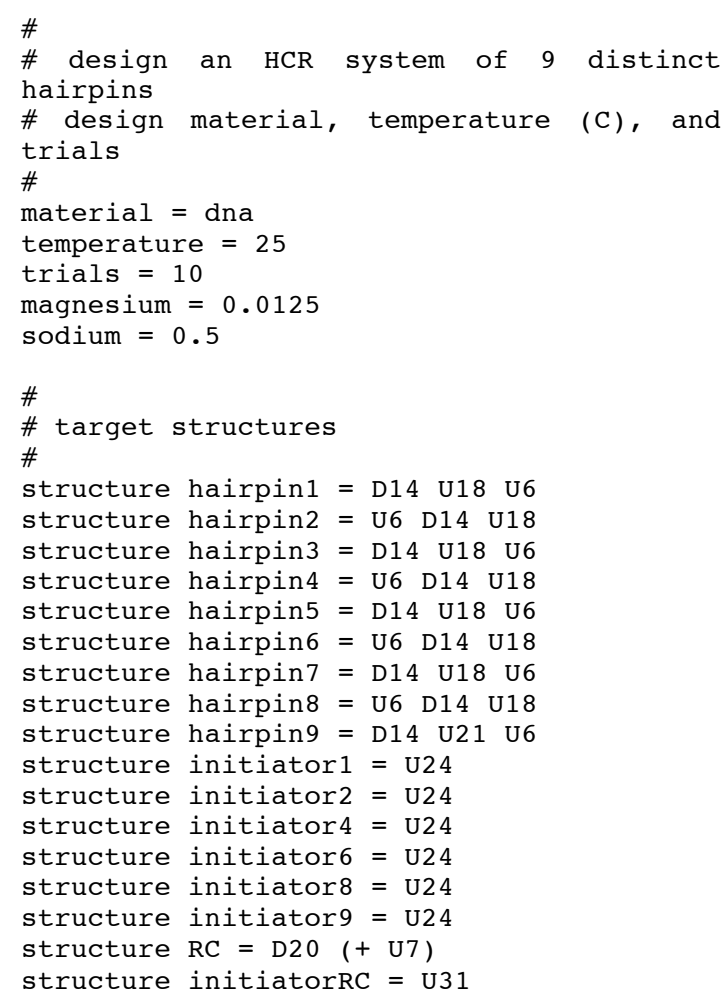




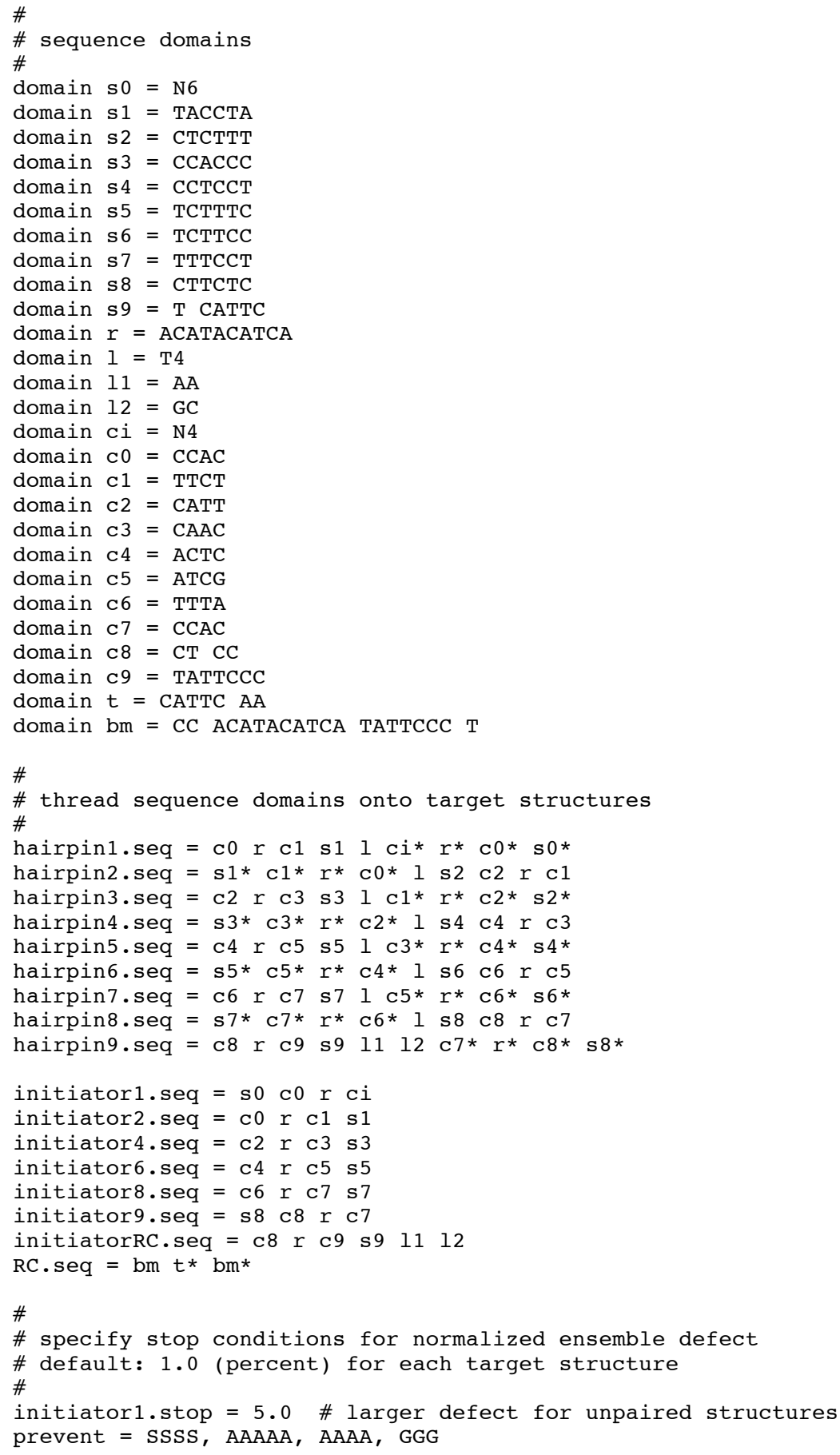


Script A.2: A system of $2^{\text {nd }}$ order chemical reactions to solve linear cascade DNA hybridization chain reactions.

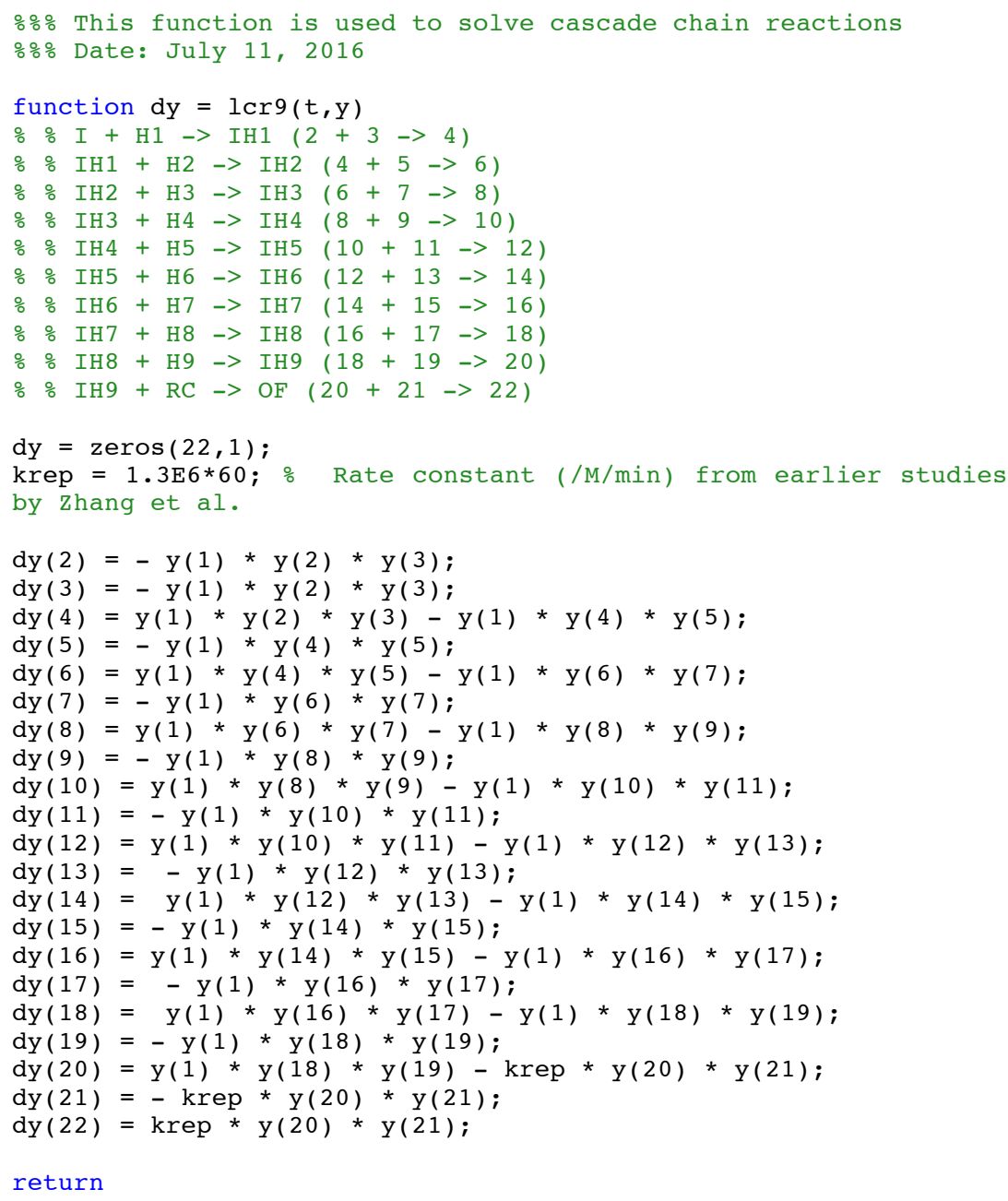




\section{Script A.3: An error function to determine the rate constant using the least- squares method.}

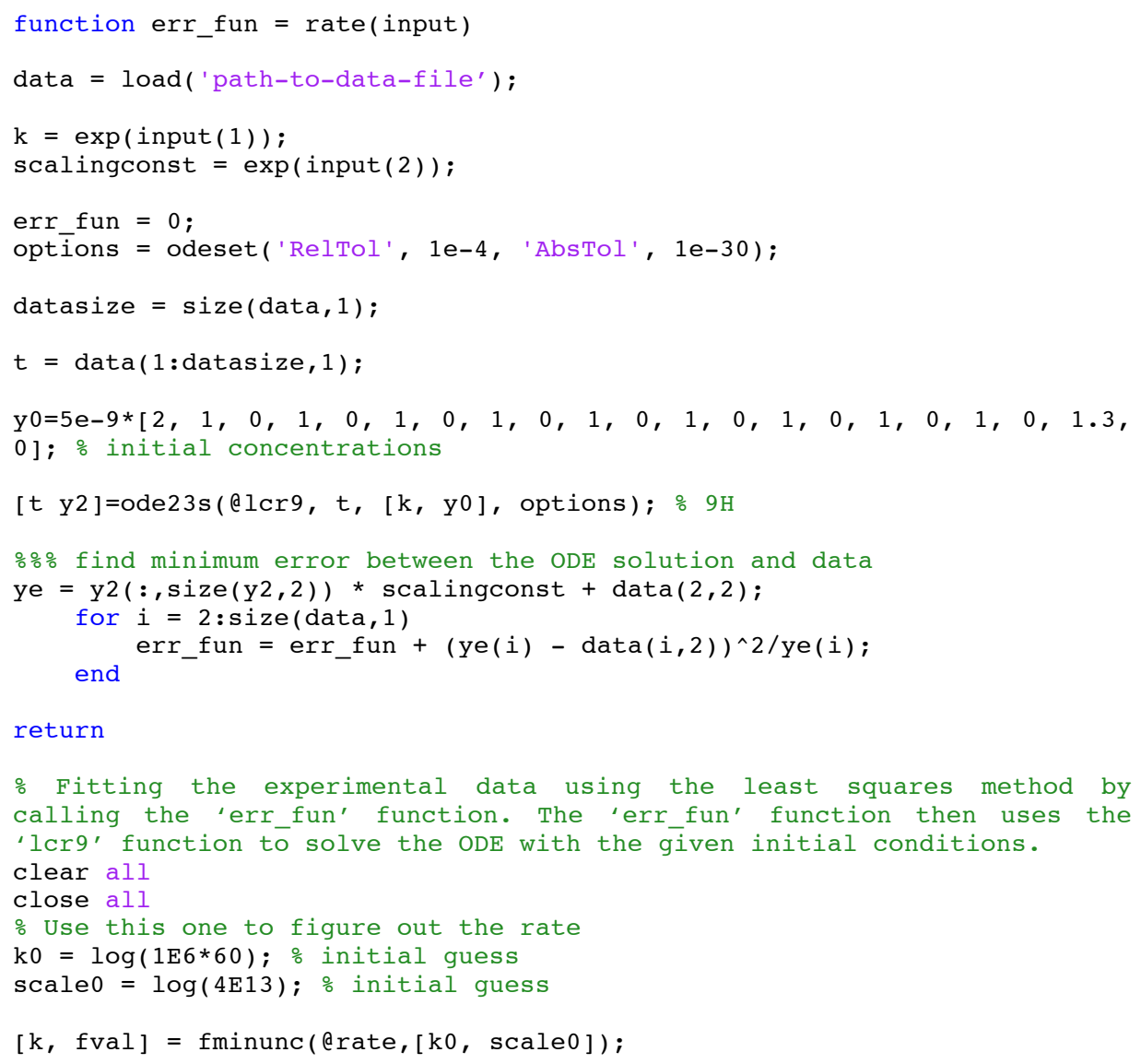


Table A.1: A list of staple strands to form DNA origami rectangle.

\begin{tabular}{|c|l|c|}
\hline $\begin{array}{c}\text { Strand } \\
\mathbf{(} \boldsymbol{)}\end{array}$ & \multicolumn{1}{|c|}{ Sequence } & $\begin{array}{c}\text { Length } \\
\text { (nt) }\end{array}$ \\
\hline $\mathbf{1}$ & $\begin{array}{l}\text { AATAATAATTTTATAGGAACCCATGTACAGGGATAG } \\
\text { CAAGCCCA }\end{array}$ & 44 \\
\hline $\mathbf{2}$ & CAGCGAAATTTTTTTTTCACGTTGAAAGAATTGCG & 36 \\
\hline $\mathbf{3}$ & CGCCTGATTTTTGACAGCATCGGAACGAACCCTCAG & 36 \\
\hline $\mathbf{4}$ & GAATAAGGTTTTAAATTGTGTCGAAATCTGTATCAT & 36 \\
\hline $\mathbf{5}$ & CATTCAACTTTTCTTGCCCTGACGAGAACATTCAGT & 36 \\
\hline $\mathbf{6}$ & AAACAGTTTTTTAATGCAGATACATAAGAATACCA & 36 \\
\hline $\mathbf{7}$ & TTTTTGCGTTTTCAGAAAACGAGAATGAAATGCTTT & 36 \\
\hline $\mathbf{8}$ & TCAATTCTTTTTGATGGCTTAGAGCTTAAGAGGTCA & 36 \\
\hline $\mathbf{9}$ & AGGTAAAGTTTTACTAATAGTAGTAGCAAGGTGGCA & 36 \\
\hline $\mathbf{1 0}$ & AGAAAAGCTTTTATTCAAAAGGGTGAGATAATGTGT & 36 \\
\hline $\mathbf{1 1}$ & GATTGACCTTTTCCCAAAAACAGGAAGATGATAATC & 36 \\
\hline $\mathbf{1 2}$ & CAGCTGGCTTTTGTAATGGGATAGGTCAAAACGGCG & 36 \\
\hline $\mathbf{1 3}$ & GCATAAAGTTTTGAAAGGGGGATGTGCTTATTACGC & 36 \\
\hline $\mathbf{1 4}$ & GAGTTGCATTTTTGTAAAGCCTGGGGTGAGCCGGAA & 36 \\
\hline $\mathbf{1 5}$ & ACCCAAATTTTTGCAAGCGGTCCACGCTCCCTGAGA & 36 \\
\hline $\mathbf{1 6}$ & TTTTCAAGTTTTTTGGGGTCGAACCATC & 28 \\
\hline $\mathbf{1 7}$ & CTCAGAGCCACCACCCTCATTTTCCGTAACAC & 32 \\
\hline $\mathbf{1 8}$ & TGAGTTTCAAAGGAACAACTAAAGATCTCCAA & 32 \\
\hline $\mathbf{1 9}$ & AAAAAAGGCTTTTGCGGGATCGTCGGGTAGCA & 32 \\
\hline $\mathbf{2 0}$ & ACGGCTACAAGTACAACGGAGATTCGCGACCT & 32 \\
\hline $\mathbf{2 1}$ & GCTCCATGACGTAACAAAGCTGCTACACCAGA & 32 \\
\hline $\mathbf{2 2}$ & ACGAGTAGATCAGTTGAGATTTAGCGCCAAAA & 32 \\
\hline $\mathbf{2 3}$ & GGAATTACCATTGAATCCCCCTCACCATAAAT & 32 \\
\hline $\mathbf{2 4}$ & CAAAAATCATTGCTCCTTTTGATAATTGCTGA & 32 \\
\hline $\mathbf{2 5}$ & ATATAATGGGGGCGCGAGCTGAAATTAACATC & 32 \\
\hline $\mathbf{2 6}$ & CAATAAATAAATGCAATGCCTGAGAAGGCCGG & 32 \\
\hline $\mathbf{2 7}$ & AGACAGTCTCATATGTACCCCGGTTTGTATAA & 32 \\
\hline $\mathbf{2 8}$ & GCAAATATGATTCTCCGTGGGAACCGTTGGTG & 32 \\
\hline
\end{tabular}




\begin{tabular}{|l|l|l|}
\hline $\mathbf{2 9}$ & TAGATGGGGTGCGGGCCTCTTCGCGCAAGGCG & 32 \\
\hline $\mathbf{3 0}$ & ATTAAGTTTTCCACACAACATACGCCTAATGA & 32 \\
\hline $\mathbf{3 1}$ & GTGAGCTAGCCCTTCACCGCCTGGGGTTTGCC & 32 \\
\hline $\mathbf{3 2}$ & CCAGCAGGCGATGGCCCACTACGTGAGGTGCC & 32 \\
\hline $\mathbf{3 3}$ & GAGAATAGGTCACCAGTACAAACTCCGCCACC & 32 \\
\hline $\mathbf{3 4}$ & AAAGGCCGCTCCAAAAGGAGCCTTAGCGGAGT & 32 \\
\hline $\mathbf{3 5}$ & GCGAAACAAGAGGCTTTGAGGACTAGGGAGTT & 32 \\
\hline $\mathbf{3 6}$ & CCAAATCATTACTTAGCCGGAACGTACCAAGC & 32 \\
\hline $\mathbf{3 7}$ & AAAGATTCTAAATTGGGCTTGAGATTCATTAC & 32 \\
\hline $\mathbf{3 8}$ & TAAATATTGAGGCATAGTAAGAGCACAGGTAG & 32 \\
\hline $\mathbf{3 9}$ & TACCTTTAAGGTCTTTACCCTGACAATCGTCA & 32 \\
\hline $\mathbf{4 0}$ & TTTCATTTCTGTAGCTCAACATGTTTAGAGAG & 32 \\
\hline $\mathbf{4 1}$ & TATATTTTCATACAGGCAAGGCAAAGCTATAT & 32 \\
\hline $\mathbf{4 2}$ & CATGTCAAAAATCACCATCAATATAACCCTCA & 32 \\
\hline $\mathbf{4 3}$ & ACCCGTCGTTAAATTGTAAACGTTAAAACTAG & 32 \\
\hline $\mathbf{4 4}$ & GGCGATCGCGCATCGTAACCGTGCGAGTAACA & 32 \\
\hline $\mathbf{4 5}$ & GCTCACAAGGGTAACGCCAGGGTTTTGGGAAG & 32 \\
\hline $\mathbf{4 6}$ & AGCTGATTACTCACATTAATTGCGTGTTATCC & 32 \\
\hline $\mathbf{4 7}$ & TATCAGGGCGAAAATCCTGTTTGACGGGCAAC & 32 \\
\hline $\mathbf{4 8}$ & GTAAAGCACTAAATCGGAACCCTAAAACCGTC & 32 \\
\hline $\mathbf{4 9}$ & CCCTCAGAACCGCCACCCTCAGAAACAACGCC & 32 \\
\hline $\mathbf{5 0}$ & TGTAGCATAACTTTCAACAGTTTCTAATTGTA & 32 \\
\hline $\mathbf{5 1}$ & TCGGTTTAGGTCGCTGAGGCTTGCAAAGACTT & 32 \\
\hline $\mathbf{5 2}$ & TTTCATGATGACCCCCAGCGATTAAGGCGCAG & 32 \\
\hline $\mathbf{5 3}$ & ACGGTCAATGACAAGAACCGGATATGGTTTAA & 32 \\
\hline $\mathbf{5 4}$ & TTTCAACTACGGAACAACATTATTAACACTAT & 32 \\
\hline $\mathbf{5 5}$ & CATAACCCGCGTCCAATACTGCGGTATTATAG & 32 \\
\hline $\mathbf{5 6}$ & TCAGAAGCCTCCAACAGGTCAGGATTTAAATA & 32 \\
\hline $\mathbf{5 7}$ & TGCAACTAGGTCAATAACCTGTTTAGAATTAG & 32 \\
\hline $\mathbf{5 8}$ & CAAAATTAGGATAAAAATTTTTAGGATATTCA & 32 \\
\hline $\mathbf{5 9}$ & ACCGTTCTGATGAACGGTAATCGTAATATTTT & 32 \\
\hline $\mathbf{6 0}$ & GTTAAAATAACATTAAATGTGAGCATCTGCCA & 32 \\
\hline
\end{tabular}




\begin{tabular}{|c|l|l|}
\hline $\mathbf{6 1}$ & GTTTGAGGTCAGGCTGCGCAACTGTTCCCAGT & 32 \\
\hline $\mathbf{6 2}$ & CACGACGTGTTTCCTGTGTGAAATTTGCGCTC & 32 \\
\hline $\mathbf{6 3}$ & ACTGCCCGCTTTTCACCAGTGAGATGGTGGTT & 32 \\
\hline $\mathbf{6 4}$ & CCGAAATCAACGTCAAAGGGCGAAAAGGGAGC & 32 \\
\hline $\mathbf{6 5}$ & TGCTAAACTCCACAGACAGCCCTCTACCGCCA & 32 \\
\hline $\mathbf{6 6}$ & ATATATTCTCAGCTTGCTTTCGAGTGGGATTT & 32 \\
\hline $\mathbf{6 7}$ & CTCATCTTGGAAGTTTCCATTAAACATAACCG & 32 \\
\hline $\mathbf{6 8}$ & AGTAATCTTCATAAGGGAACCGAACTAAAACA & 32 \\
\hline $\mathbf{6 9}$ & ACGAACTATTAATCATTGTGAATTTCATCAAG & 32 \\
\hline $\mathbf{7 0}$ & ACTGGATATCGTTTACCAGACGACTTAATAAA & 32 \\
\hline $\mathbf{7 1}$ & GAAGCAAAAAAGCGGATTGCATCAATGTTTAG & 32 \\
\hline $\mathbf{7 2}$ & TCGCAAATAAGTACGGTGTCTGGACCAGACCG & 32 \\
\hline $\mathbf{7 3}$ & CAACGCAAAGCAATAAAGCCTCAGGATACATT & 32 \\
\hline $\mathbf{7 4}$ & AGAGAATCAGCTGATAAATTAATGCTTTATTT & 32 \\
\hline $\mathbf{7 5}$ & CTTTCATCTCGCATTAAATTTTTGAGCAAACA & 32 \\
\hline $\mathbf{7 6}$ & TTCGCCATGGACGACGACAGTATCGTAGCCAG & 32 \\
\hline $\mathbf{7 7}$ & TCATAGCTTGTAAAACGACGGCCAAAGCGCCA & 32 \\
\hline $\mathbf{7 8}$ & TGGTTTTTCTTTCCAGTCGGGAAAAATCATGG & 32 \\
\hline $\mathbf{7 9}$ & TGGACTCCGGCAAAATCCCTTATACGCCAGGG & 32 \\
\hline $\mathbf{8 0}$ & CCCCGATTTAGAGCTTGACGGGGAAAAGAACG & 32 \\
\hline $\mathbf{8 1}$ & TATCACCGTACTCAGGAGGTTTAGATAGTTAG & 32 \\
\hline $\mathbf{8 2}$ & CGTAACGAAAATGAATTTTCTGTAGTGAATTT & 32 \\
\hline $\mathbf{8 3}$ & CTTAAACAACAACCATCGCCCACGCGGGTAAA & 32 \\
\hline $\mathbf{8 4}$ & ATACGTAAGAGGCAAAAGAATACACTGACCAA & 32 \\
\hline $\mathbf{8 5}$ & CTTTGAAAATAGGCTGGCTGACCTACCTTATG & 32 \\
\hline $\mathbf{8 6}$ & CGATTTTAGGAAGAAAAATCTACGGATAAAAA & 32 \\
\hline $\mathbf{8 7}$ & CCAAAATAAGGGGGTAATAGTAAAAAAAGATT & 32 \\
\hline $\mathbf{8 8}$ & AAGAGGAACGAGCTTCAAAGCGAAAGTTTCAT & 32 \\
\hline $\mathbf{8 9}$ & TCCATATATTTAGTTTGACCATTAAGCATAAA & 32 \\
\hline $\mathbf{9 0}$ & GCTAAATCCTTTTGCGGGAGAAGCCCGGAGAG & 32 \\
\hline $\mathbf{9 1}$ & GGTAGCTATTGCCTGAGAGTCTGGTTAAATCA & 32 \\
\hline $\mathbf{9 2}$ & GCTCATTTCGCGTCTGGCCTTCCTGGCCTCAG & 32 \\
\hline
\end{tabular}




\begin{tabular}{|c|l|l|}
\hline $\mathbf{9 3}$ & GAAGATCGTGCCGGAAACCAGGCAGTGCCAAG & 32 \\
\hline $\mathbf{9 4}$ & CTTGCATGCCGAGCTCGAATTCGTCCTGTCGT & 32 \\
\hline $\mathbf{9 5}$ & GCCAGCTGCGGTTTGCGTATTGGGAATCAAAA & 32 \\
\hline $\mathbf{9 6}$ & GAATAGCCACAAGAGTCCACTATTAAGCCGGC & 32 \\
\hline $\mathbf{9 7}$ & ACGTTAGTTCTAAAGTTTTGTCGTGATACAGG & 32 \\
\hline $\mathbf{9 8}$ & CAATGACAGCTTGATACCGATAGTCTCCCTCA & 32 \\
\hline $\mathbf{9 9}$ & AAACGAAATGCCACTACGAAGGCAGCCAGCAA & 32 \\
\hline $\mathbf{1 0 0}$ & CCAGGCGCGAGGACAGATGAACGGGTAGAAAA & 32 \\
\hline $\mathbf{1 0 1}$ & GGACGTTGAGAACTGGCTCATTATGCGCTAAT & 32 \\
\hline $\mathbf{1 0 2}$ & TTTGCCAGGCGAGAGGCTTTTGCAATCCTGAA & 32 \\
\hline $\mathbf{1 0 3}$ & TTTTAATTGCCCGAAAGACTTCAACAAGAACG & 32 \\
\hline $\mathbf{1 0 4}$ & CGAGTAGAACAGTTGATTCCCAATATTTAGGC & 32 \\
\hline $\mathbf{1 0 5}$ & CTGTAATAGGTTGTACCAAAAACACAAATATA & 32 \\
\hline $\mathbf{1 0 6}$ & TCAGGTCATTTTTGAGAGATCTACCCTTGCTT & 32 \\
\hline $\mathbf{1 0 7}$ & AAATAATTTTTAACCAATAGGAACAACAGTAC & 32 \\
\hline $\mathbf{1 0 8}$ & GCTTCTGGCACTCCAGCCAGCTTTACATTATC & 32 \\
\hline $\mathbf{1 0 9}$ & CCCGGGTACCTGCAGGTCGACTCTCAAATATC & 32 \\
\hline $\mathbf{1 1 0}$ & GGGAGAGGCATTAATGAATCGGCCACCTGAAA & 32 \\
\hline $\mathbf{1 1 1}$ & AGTTTGGACGAGATAGGGTTGAGTGTAATAAC & 32 \\
\hline $\mathbf{1 1 2}$ & GAACGTGGCGAGAAAGGAAGGGAATGCGCCGC & 32 \\
\hline $\mathbf{1 1 3}$ & AGGGTTGATATAAGTATAGCCCGGAATAGGTG & 32 \\
\hline $\mathbf{1 1 4}$ & AGTGTACTATACATGGCTTTTGATCTTTCCAG & 32 \\
\hline $\mathbf{1 1 5}$ & GAGCCGCCCCACCACCGGAACCGCTGCGCCGA & 32 \\
\hline $\mathbf{1 1 6}$ & AATCACCACCATTTGGGAATTAGACCAACCTA & 32 \\
\hline $\mathbf{1 1 7}$ & TACATACACAGTATGTTAGCAAACTGTACAGA & 32 \\
\hline $\mathbf{1 1 8}$ & ATCAGAGAGTCAGAGGGTAATTGAACCAGTCA & 32 \\
\hline $\mathbf{1 1 9}$ & TCTTACCAACCCAGCTACAATTTTAAAGAAGT & 32 \\
\hline $\mathbf{1 2 0}$ & GGTATTAATCTTTCCTTATCATTCATATCGCG & 32 \\
\hline $\mathbf{1 2 1}$ & AGAGGCATACAACGCCAACATGTATCTGCGAA & 32 \\
\hline $\mathbf{1 2 2}$ & TTTTAGTTCGCGAGAAAACTTTTTTTATGACC & 32 \\
\hline $\mathbf{1 2 3}$ & CTGTAAATATATGTGAGTGAATAAAAAGGCTA & 32 \\
\hline $\mathbf{1 2 4}$ & CTTTTACACAGATGAATATACAGTGCCATCAA & 32 \\
\hline
\end{tabular}




\begin{tabular}{|c|l|l|}
\hline $\mathbf{1 2 5}$ & ATTTTGCGTTTAAAAGTTTGAGTACCGGCACC & 32 \\
\hline $\mathbf{1 2 6}$ & AAACCCTCTCACCTTGCTGAACCTAGAGGATC & 32 \\
\hline $\mathbf{1 2 7}$ & GCGTAAGAAGATAGAACCCTTCTGAACGCGCG & 32 \\
\hline $\mathbf{1 2 8}$ & ATCACTTGAATACTTCTTTGATTAGTTGTTCC & 32 \\
\hline $\mathbf{1 2 9}$ & TAAGCGTCGGTAATAAGTTTTAACCCGTCGAG & 32 \\
\hline $\mathbf{1 3 0}$ & AACCAGAGACCCTCAGAACCGCCACGTTCCAG & 32 \\
\hline $\mathbf{1 3 1}$ & GACTTGAGGTAGCACCATTACCATATCACCGG & 32 \\
\hline $\mathbf{1 3 2}$ & TTATTACGTAAAGGTGGCAACATACCGTCACC & 32 \\
\hline $\mathbf{1 3 3}$ & TGAACAAAGATAACCCACAAGAATAAGACTCC & 32 \\
\hline $\mathbf{1 3 4}$ & TATTTTGCACGCTAACGAGCGTCTGAACACCC & 32 \\
\hline $\mathbf{1 3 5}$ & ATCGGCTGACCAAGTACCGCACTCTTAGTTGC & 32 \\
\hline $\mathbf{1 3 6}$ & CATATTTATTTCGAGCCAGTAATAAATCAATA & 32 \\
\hline $\mathbf{1 3 7}$ & ACAAAGAAAATTTCATCTTCTGACAGAATCGC & 32 \\
\hline $\mathbf{1 3 8}$ & AAATCAATCGTCGCTATTAATTAAATCGCAAG & 32 \\
\hline $\mathbf{1 3 9}$ & TTTAACGTTCGGGAGAAACAATAACAGTACAT & 32 \\
\hline $\mathbf{1 4 0}$ & TTATTAATGAACAAAGAAACCACCTTTTCAGG & 32 \\
\hline $\mathbf{1 4 1}$ & CTAAAGCAAATCAATATCTGGTCACCCGAACG & 32 \\
\hline $\mathbf{1 4 2}$ & GCCAACAGATACGTGGCACAGACATGAAAAAT & 32 \\
\hline $\mathbf{1 4 3}$ & GTTGTAGCCCTGAGTAGAAGAACTACATTCTG & 32 \\
\hline $\mathbf{1 4 4}$ & TACAGGGCGCGTACTATGGTTGCTAATTAACC & 32 \\
\hline $\mathbf{1 4 5}$ & TGCTCAGTACCAGGCGGATAAGTGGGGGTCAG & 32 \\
\hline $\mathbf{1 4 6}$ & TGCCTTGACAGTCTCTGAATTTACCCCTCAGA & 32 \\
\hline $\mathbf{1 4 7}$ & GCCACCACTCTTTTCATAATCAAATAGCAAGG & 32 \\
\hline $\mathbf{1 4 8}$ & CCGGAAACTAAAGGTGAATTATCATAAAAGAA & 32 \\
\hline $\mathbf{1 4 9}$ & ACGCAAAGAAGAACTGGCATGATTTGAGTTAA & 32 \\
\hline $\mathbf{1 5 0}$ & GCCCAATAGACGGGAGAATTAACTTTCCAGAG & 32 \\
\hline $\mathbf{1 5 1}$ & CCTAATTTAAGCCTTAAATCAAGAATCGAGAA & 32 \\
\hline $\mathbf{1 5 2}$ & CAAGCAAGCGAGCATGTAGAAACCAGAGAATA & 32 \\
\hline $\mathbf{1 5 3}$ & TAAAGTACCAGTAGGGCTTAATTGCTAAATTT & 32 \\
\hline $\mathbf{1 5 4}$ & AATGGTTTTGCTGATGCAAATCCATTTTCCCT & 32 \\
\hline $\mathbf{1 5 5}$ & TAGAATCCCCTTTTTTAATGGAAACGGATTCG & 32 \\
\hline $\mathbf{1 5 6}$ & CCTGATTGAAAGAAATTGCGTAGAAGAAGGAG & 32 \\
\hline
\end{tabular}




\begin{tabular}{|l|l|l|}
\hline $\mathbf{1 5 7}$ & CGGAATTACGTATTAAATCCTTTGGTTGGCAA & 32 \\
\hline $\mathbf{1 5 8}$ & ATCAACAGGAGAGCCAGCAGCAAAATATTTT & 32 \\
\hline $\mathbf{1 5 9}$ & GAATGGCTACCAGTAATAAAAGGGCAAACTAT & 32 \\
\hline $\mathbf{1 6 0}$ & CGGCCTTGGTCTGTCCATCACGCATTGACGAG & 32 \\
\hline $\mathbf{1 6 1}$ & GGAAAGCGGTAACAGTGCCCGTATCGGGGTTT & 32 \\
\hline $\mathbf{1 6 2}$ & GTTTGCCACCTCAGAGCCGCCACCGCCAGAAT & 32 \\
\hline $\mathbf{1 6 3}$ & TTATTCATGTCACCAATGAAACCATTATTAGC & 32 \\
\hline $\mathbf{1 6 4}$ & ATACCCAAACACCACGGAATAAGTGACGGAAA & 32 \\
\hline $\mathbf{1 6 5}$ & GCGCATTAATAAGAGCAAGAAACAATAACGGA & 32 \\
\hline $\mathbf{1 6 6}$ & AGGTTTTGGCCAGTTACAAAATAAACAGGGAA & 32 \\
\hline $\mathbf{1 6 7}$ & CTAATTTACCGTTTTTATTTTCATCTTGCGGG & 32 \\
\hline $\mathbf{1 6 8}$ & ACGCTCAACGACAAAAGGTAAAGTATCCCATC & 32 \\
\hline $\mathbf{1 6 9}$ & TATGTAAAGAAATACCGACCGTGTTAAAGCCA & 32 \\
\hline $\mathbf{1 7 0}$ & TTGAATTATTGAAAACATAGCGATTATAACTA & 32 \\
\hline $\mathbf{1 7 1}$ & ACAGAAATCTTTGAATACCAAGTTAATTTCAT & 32 \\
\hline $\mathbf{1 7 2}$ & CGACAACTTCATCATATTCCTGATCACGTAAA & 32 \\
\hline $\mathbf{1 7 3}$ & GCCACGCTTTGAAAGGAATTGAGGAAACAATT & 32 \\
\hline $\mathbf{1 7 4}$ & GTCACACGATTAGTCTTTAATGCGGCAACAGT & 32 \\
\hline $\mathbf{1 7 5}$ & GTAAAAGACTGGTAATATCCAGAAATTCACCA & 32 \\
\hline $\mathbf{1 7 6}$ & CACGTATAACGTGCTTTCCTCGTTGCCACCGA & 32 \\
\hline $\mathbf{1 7 7}$ & CCTCAAGAGAAGGATTAGGATTAGAAACAGTT & 32 \\
\hline $\mathbf{1 7 8}$ & AATGCCCCATAAATCCTCATTAAAAGAACCAC & 32 \\
\hline $\mathbf{1 7 9}$ & CACCAGAGTTCGGTCATAGCCCCCTCGATAGC & 32 \\
\hline $\mathbf{1 8 0}$ & AGCACCGTAGGGAAGGTAAATATTTTATTTTG & 32 \\
\hline $\mathbf{1 8 1}$ & TCACAATCCCGAGGAAACGCAATAATGAAATA & 32 \\
\hline $\mathbf{1 8 2}$ & GCAATAGCAGAGAATAACATAAAAACAGCCAT & 32 \\
\hline $\mathbf{1 8 3}$ & ATTATTTATTAGCGAACCTCCCGACGTAGGAA & 32 \\
\hline $\mathbf{1 8 4}$ & TCATTACCGAACAAGAAAAATAATAATTCTGT & 32 \\
\hline $\mathbf{1 8 5}$ & CCAGACGACAAATTCTTACCAGTAGATAAATA & 32 \\
\hline $\mathbf{1 8 6}$ & AGGCGTTAGGCTTAGGTTGGGTTAAGCTTAGA & 32 \\
\hline $\mathbf{1 8 7}$ & TTAAGACGATTAATTACATTTAACACAAAATC & 32 \\
\hline $\mathbf{1 8 8}$ & GCGCAGAGATATCAAAATTATTTGTATCAGAT & 32 \\
\hline
\end{tabular}




\begin{tabular}{|c|c|c|}
\hline 189 & GATGGCAAAAGTATTAGACTTTACAAGGTTAT & 32 \\
\hline 190 & CTAAAATAAGTATTAACACCGCCTCGAACTGA & 32 \\
\hline 191 & TAGCCCTATTATTTACATTGGCAGCAATATTA & 32 \\
\hline 192 & CCGCCAGCTTTTATAATCAGTGAGAGAATCAG & 32 \\
\hline 193 & ACAAACAACTGCCTATTTCGGAACCTGAGACT & 32 \\
\hline 194 & TCGGCATTCCGCCGCCAGCATTGATGATATTC & 32 \\
\hline 195 & ATTGAGGGAATCAGTAGCGACAGACGTTTTCA & 32 \\
\hline 196 & GAAGGAAAAATAGAAAATTCATATTTCAACCG & 32 \\
\hline 197 & CTTTACAGTATCTTACCGAAGCCCAGTTACCA & 32 \\
\hline 198 & GAGGCGTTTCCCAATCCAAATAAGATAGCAGC & 32 \\
\hline 199 & TAAGTCCTGCGCCCAATAGCAAGCAAGAACGC & 32 \\
\hline 200 & GCGTTATACGACAATAAACAACATACAATAGA & 32 \\
\hline 201 & TAACСТCCAATAAGAATAAACACCTATCATAT & 32 \\
\hline 202 & AAAACAAACTGAGAAGAGTCAATATACCTTTT & 32 \\
\hline 203 & AACCTACCGCGAATTATTCATTTCACATCAAG & 32 \\
\hline 204 & GGATTTAGTTCATCAATATAATCCAGGGTTAG & 32 \\
\hline 205 & AGGCGGTCTCTTTAGGAGCACTAAACATTTGA & 32 \\
\hline 206 & GAAATGGAAAACATCGCCATTAAACAGAGGTG & 32 \\
\hline 207 & AGAAGTGTCATTGCAACAGGAAAAAATCGTCT & 32 \\
\hline 208 & AGCGGGAGCTAAACAGGAGGCCGAGAATCCTG & 32 \\
\hline 209 & TTTTTGAAAGTATTAAGAGGCTATTATT & 28 \\
\hline 210 & CTGAAACATTTTGTCAGACGATTGGCCTCAGGAGGT & 36 \\
\hline 211 & TGAGGCAGTTTTGCGTCAGACTGTAGCGATCAAGTT & 36 \\
\hline 212 & TGCCTTTATTTTAGACAAAAGGGCGACAGGTTTACC & 36 \\
\hline 213 & AGCGCCAATTTTGCAGATAGCCGAACAATTTTTAAG & 36 \\
\hline 214 & $\begin{array}{l}\text { AAAAGTAATTTTAACGTCAAAAATGAAAAAACGAT } \\
\text { T }\end{array}$ & 36 \\
\hline 215 & TTTTGTTTTTTTGCTTATCCGGTATTCTAAATCAGA & 36 \\
\hline 216 & TATAGAAGTTTTACGCGCCTGTTTATCAGTTCAGCT & 36 \\
\hline 217 & AATGCAGATTTTGAAAAAGCCTGTTTAGGGAATCAT & 36 \\
\hline 218 & AATTACTATTTTCATAGGTCTGAGAGACGTGAATTT & 36 \\
\hline 219 & ATCAAAATTTTTGAAGATGATGAAACAAAATTACCT & 36 \\
\hline
\end{tabular}




\begin{tabular}{|c|l|c|}
\hline $\mathbf{2 2 0}$ & GAGCAAAATTTTACTTCTGAATAATGGATGATTGTT & 36 \\
\hline $\mathbf{2 2 1}$ & TGGATTATTTTTGCCGTCAATAGATAATCAACTAAT & 36 \\
\hline $\mathbf{2 2 2}$ & AGATTAGATTTTCCAGCAGAAGATAAAAAATACCG & \\
\hline $\mathbf{2 2 3}$ & ACGAACCATTTTCTACATTTTGACGCTCACGCTCAT & 36 \\
\hline $\mathbf{2 2 4}$ & $\begin{array}{l}\text { GGAAATACTTTTCAGGAACGGTACGCCATTAAAGG } \\
\text { GATTTTAGA }\end{array}$ & 44 \\
\hline
\end{tabular}




\section{Appendix B - Determination of Streptavidin-Biotin Binding Efficiency}

AFM imaging of binding efficiency between biotin-labeled DNA strand

and streptavidin molecules on the surface of DNA origami rectangles are shown

in Figure B.1. Various concentrations of streptavidin (STV) were performed while keeping the same concentration of biotin-labeled DNA origami rectangle solution in order to determine an optimal stoichiometric ratio between biotin and streptavidin molecules. For $5 \mathrm{nM}$ solution of biotin-labeled DNA origami rectangles, the optimal concentration range of streptavidin is between $55 \mathrm{nM}$ to $121 \mathrm{nM}$.
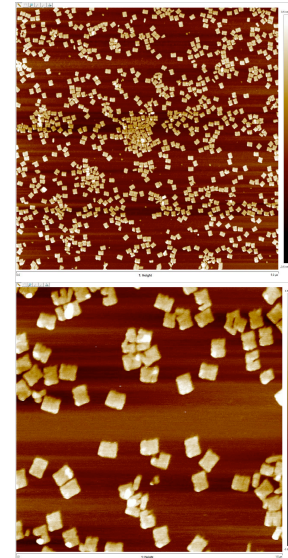

(A)
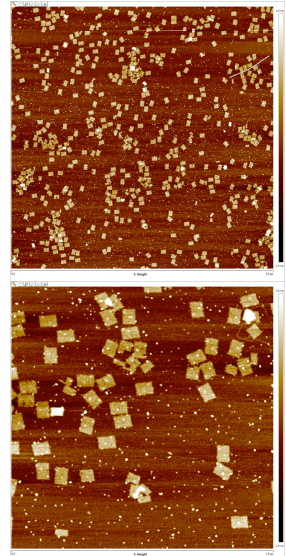

(B)
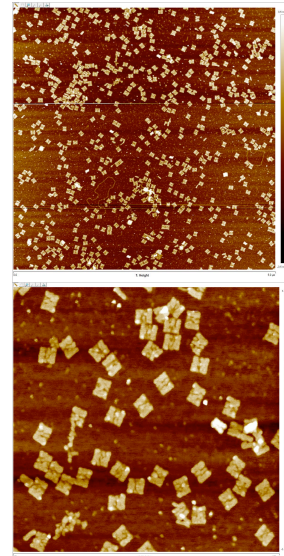

(C)
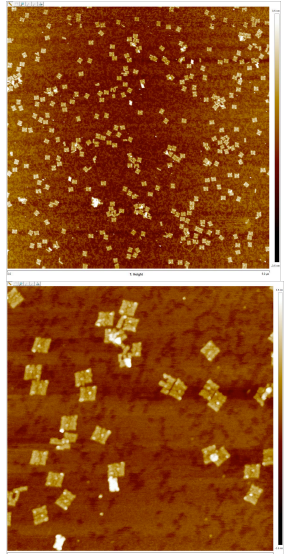

(D)
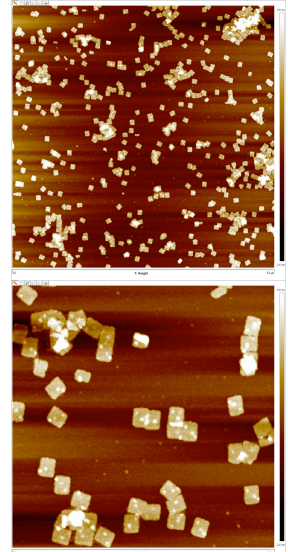

(E)

Control $0 \mathrm{nM} \quad$ STV $443 \mathrm{nM}$

STV $55 \mathrm{nM}$

STV $15 \mathrm{nM}$ 
Figure B.1: DNA origami rectangles labeled with biotinylated oligomers: (A) 5 nM DNA rectangle sample without streptavidin, (B) $5 \mathrm{nM}$ DNA rectangle sample with $443 \mathrm{nM}$ streptavidin, (C) $5 \mathrm{nM}$ DNA rectangle sample with $121 \mathrm{nM}$ streptavidin, (D) $5 \mathrm{nM}$ DNA rectangle sample with $55 \mathrm{nM}$ streptavidin, (E) $5 \mathrm{nM}$ DNA rectangle sample with $15 \mathrm{nM}$ streptavidin.

To determine the binding yield between biotin-labeled DNA and streptavidin on the surface of DNA origami rectangle, a total of 63 DNA origami rectangles were analyzed. The maximum available biotins on the surface of DNA origami rectangle is 3 . After mixing, the solution was allowed to sit at room temperature for 30 minutes prior to AFM imaging. A total of 29 DNA origami rectangles contains the maximum of three streptavidin molecules. This translates to roughly $46 \%$ binding efficiency. The remainder DNA origami rectangles contain 0,1 , and 2 streptavidin molecules (roughly $54 \%$ ).

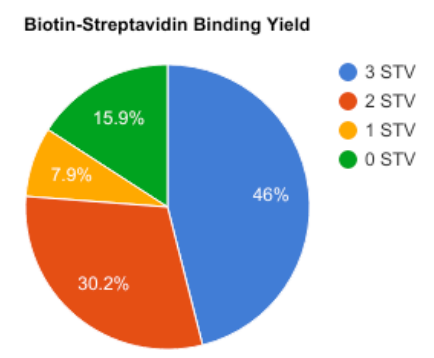

Figure B.2: Binding yield between biotin and streptavidin on the surface of DNA origami rectangle.

For a single binding event between biotin and streptavidin on DNA origami rectangles, the binding yield was determined to be roughly $81 \%$ - a total 
of 37 DNA origami rectangles was analyzed and 30 DNA origami rectangles contained a designated streptavidin molecule.

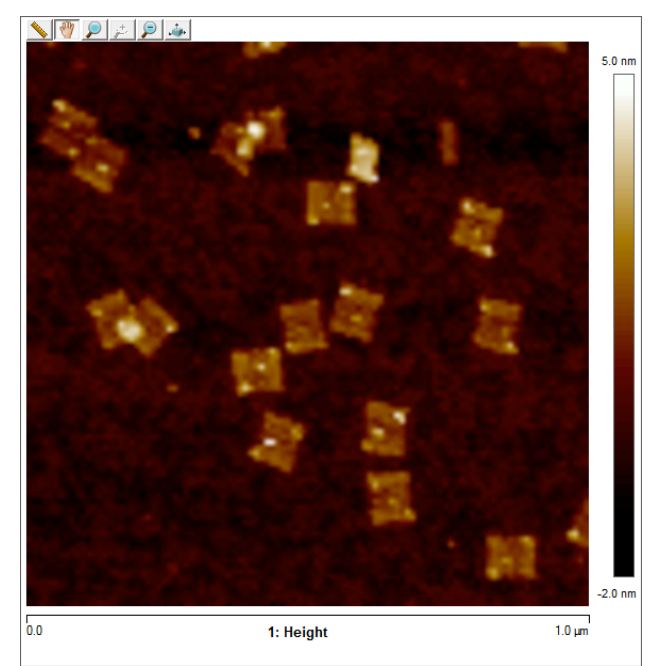

(A)

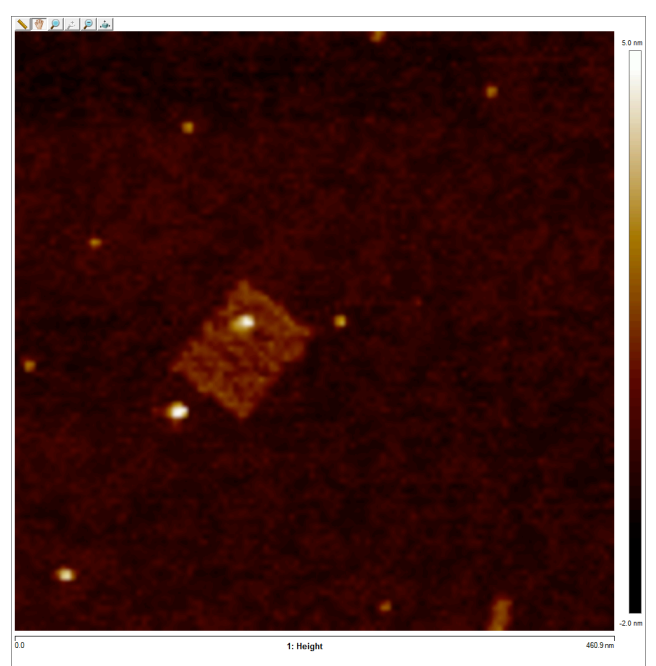

(B)

Figure B.3: Binding efficiency between biotin and streptavidin molecules. Maximum available spots for binding (A) 3 molecules and (B) 1 molecule. 


\section{Appendix C - Sequence Design for Constructing Redundancy and Reversible DNA Gates}

To test the effect of the hairpin loop, all domains are designed to have $5 \mathrm{nt}$ except the l-domain which is varying from 5 to $10 \mathrm{nt}$. In the presence of the initiator, the pair of DNA hairpins do not hybridize if the strand displacement process results in a weaker bond in comparison to the bond of the incumbent stem. For instance, the toehold domain is $5 \mathrm{nt}$. The stem consists of two domains ( $a$ and $b$ ) which are $5 \mathrm{nt}$ in length. The initiator consists of two domains ( $t$ and $a$ ) which are $5 \mathrm{nt}$ in length. Suppose the loop constraint may hinder the hybridization process, the NUPACK simulation data indicate that changing the $l$ domain does not improve or hinder the hybridization. By increasing the strength of the strand displacement process to have 5 additional base pairs, the pair of DNA hairpins hybridize and form a desired complex and in the presence of the initiator. For instance, before the strand displacement process, the stem consists of 10 base pairs. After the strand displacement process, the stem and the invading strand form 15 base pairs.

To reverse the forward reaction, a domain $\mathrm{g}_{\mathrm{i}}$ is incorporated and acts as a toehold domain for a destabilizing hairpin $\left(\mathrm{DH}_{\mathrm{i}}\right)$ to disrupt the duplex between hairpin $\mathrm{H}_{i}$ and $\mathrm{H}_{\mathrm{i}+1}$. The addition of $\mathrm{g}_{\mathrm{i}}$ does not cause the leak in the forward 
direction based on NUPACK analysis. Only when the hairpins $\mathrm{H}_{\mathrm{i}}$ and $\mathrm{H}_{\mathrm{i}+1}$ hybridized, then $\mathrm{DH}_{\mathrm{i}}$ hairpin can hybridize to the $\mathrm{g}_{\mathrm{i}}$ domain as shown in Figure C.1. In the absence of the initiator, neither $\mathrm{DH}_{\mathrm{i}}$ hairpin or $\mathrm{RH}_{\mathrm{i}}$ hairpin hybridizes to $\mathrm{H}_{\mathrm{i}}$ hairpin. In the presence of the initiator, $\mathrm{H}_{\mathrm{i}}$ hairpin is activated by the initiator and subsequently hybridizes to $\mathrm{H}_{\mathrm{i}+1}$ hairpin. $\mathrm{DH}_{\mathrm{i}}$ hairpin is introduced and is programmed to disrupt the duplex between hairpins $\mathrm{H}_{\mathrm{i}}$ and $\mathrm{H}_{\mathrm{i}+1}$ to reverse the reaction. To allow hairpin $\mathrm{H}_{\mathrm{i}}$ to revert back to its original state, $\mathrm{RH}_{\mathrm{i}}$ hairpin is introduced and is programmed to hybridize to $\mathrm{DH}_{\mathrm{i}}$ hairpin. The script to generate this system is shown in Script C.1.
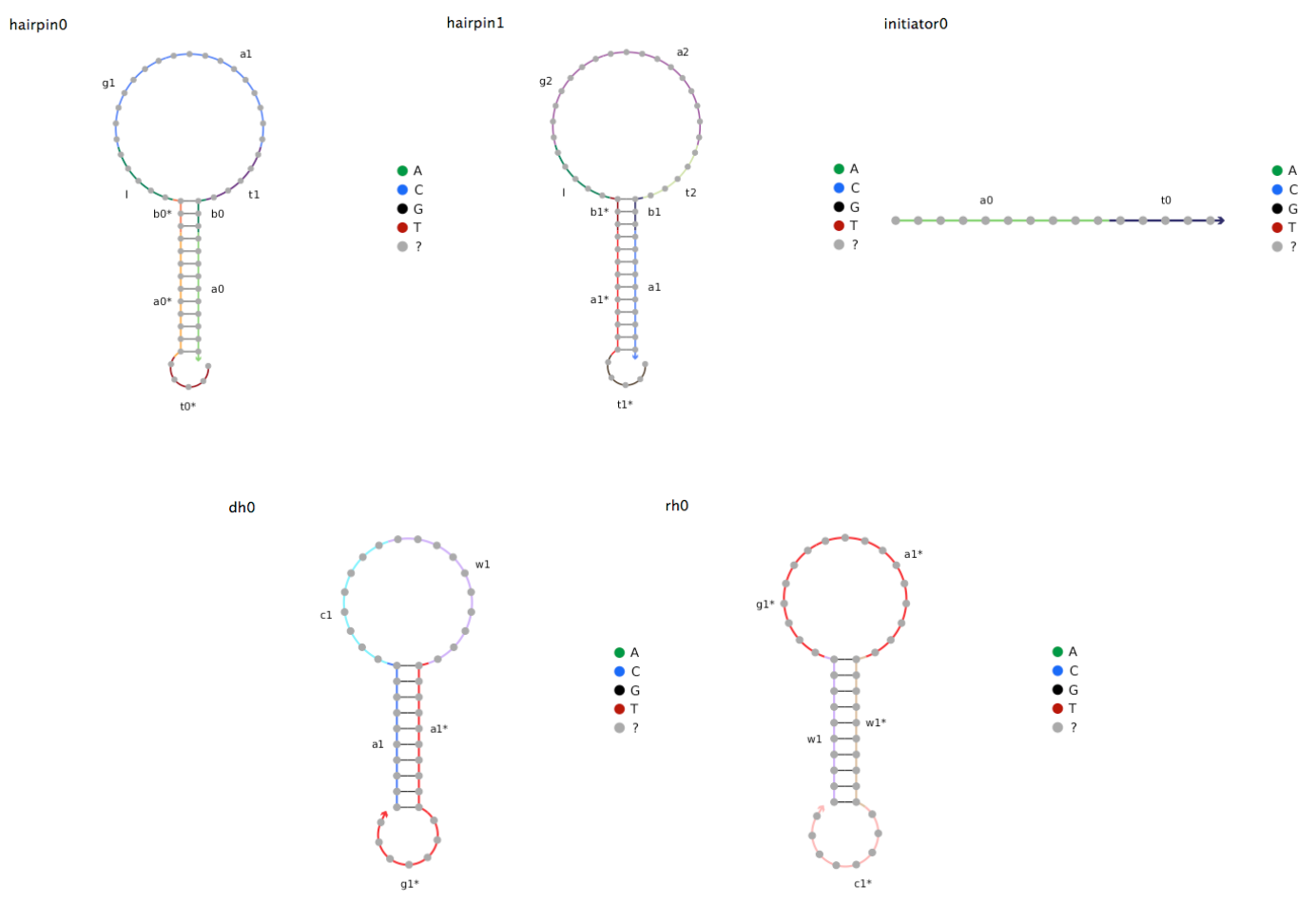


\section{Figure C.1: Programming reversible DNA hybridization reactions using DNA}

hairpins. The design consists of a pair of DNA hairpin gates that can go forward and reverse. In particular, the presence of the initiator triggers the forward reaction and the presence of the destabilizing and removing hairpins trigger the reverse reaction. Note: the length of each domain can be found in Script C.1.

\section{Script C.1: NUPACK script to construct reversible DNA hybridization reactions.}

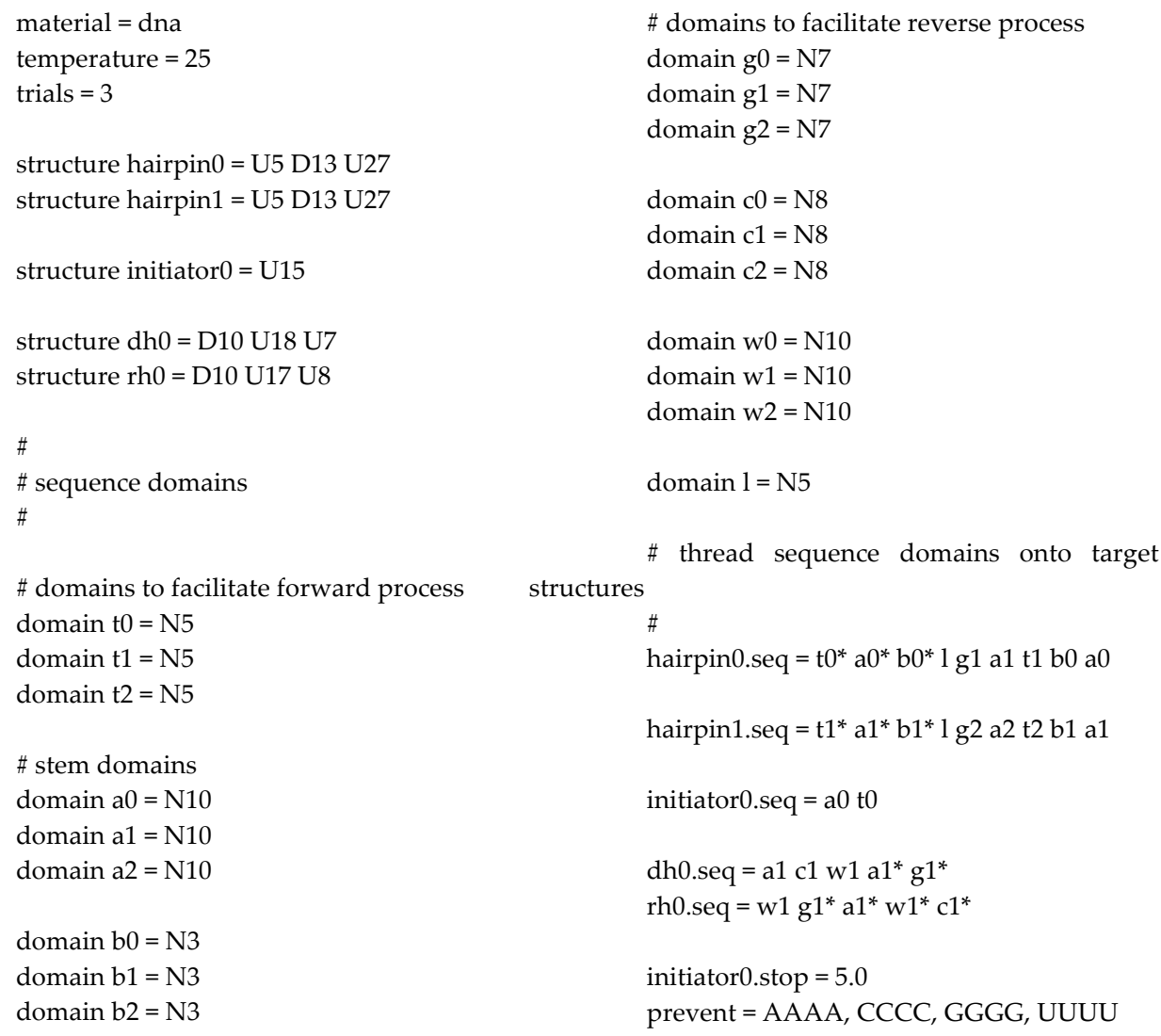




\section{Bibliography}

1 Stojanovic, M. N. Molecular computing with deoxyribozymes. Progress in nucleic acid research and molecular biology 82, 199-217, (2008).

2 Chen, Y. J., Groves, B., Muscat, R. A. \& Seelig, G. DNA nanotechnology from the test tube to the cell. Nature nanotechnology 10, 748-760, (2015).

3 Groves, B. et al. Computing in mammalian cells with nucleic acid strand exchange. Nature nanotechnology 11, 287-+, (2016).

4 Qian, L. \& Winfree, E. Scaling Up Digital Circuit Computation with DNA Strand Displacement Cascades. Science 332, 1196-1201, (2011).

5 Dalchau, N., Chandran, H., Gopalkrishnan, N., Phillips, A. \& Reif, J. Probabilistic Analysis of Localized DNA Hybridization Circuits. ACS Synth. Biol. 4, 898-913, (2015).

6 Bui, H. et al. Design and Analysis of Localized DNA Hybridization Chain Reactions. Small, 1602983-n/a, (2017).

$7 \quad$ Kelley, S. O. et al. Advancing the speed, sensitivity and accuracy of biomolecular detection using multi-length-scale engineering. Nature nanotechnology 9, 969-980, (2014).

8 Walt, D. R. Chemistry. Miniature analytical methods for medical diagnostics. Science 308, 217-219, (2005).

9 Giljohann, D. A. \& Mirkin, C. A. Drivers of biodiagnostic development. Nature 462, 461-464, (2009).

10 Crick, F. Central Dogma of Molecular Biology. Nature 227, 561-\&, (1970).

11 Seeman, N. C. DNA in a material world. Nature 421, 427-431, (2003).

12 Shih, W. M. \& Lin, C. X. Knitting complex weaves with DNA origami. Curr Opin Struc Biol 20, 276-282, (2010). 
13 Nangreave, J., Han, D. R., Liu, Y. \& Yan, H. DNA origami: a history and current perspective. Curr Opin Chem Biol 14, 608-615, (2010).

14 Seeman, N. C. Nucleic acid junctions and lattices. J Theor Biol 99, 237-247, (1982).

15 Rothemund, P. W. K. Folding DNA to create nanoscale shapes and patterns. Nature 440, 297-302, (2006).

16 Ke, Y. G., Ong, L. L., Shih, W. M. \& Yin, P. Three-Dimensional Structures Self-Assembled from DNA Bricks. Science 338, 1177-1183, (2012).

17 Wei, B., Dai, M. J. \& Yin, P. Complex shapes self-assembled from singlestranded DNA tiles. Nature 485, 623-+, (2012).

18 Williams, B. A. R., Lund, K., Liu, Y., Yan, H. \& Chaput, J. C. Selfassembled peptide nanoarrays: An approach to studying protein-protein interactions. Angew Chem Int Edit 46, 3051-3054, (2007).

19 Yang, H. et al. Metal-nucleic acid cages. Nat Chem 1, 390-396, (2009).

20 Bui, H. et al. Programmable Periodicity of Quantum Dot Arrays with DNA Origami Nanotubes. Nano letters 10, 3367-3372, (2010).

21 Voigt, N. V. et al. Single-molecule chemical reactions on DNA origami. Nature nanotechnology 5, 200-203, (2010).

22 Fu, T. J. \& Seeman, N. C. DNA Double-Crossover Molecules. BiochemistryUs 32, 3211-3220, (1993).

23 LaBean, T. H. et al. Construction, analysis, ligation, and self-assembly of DNA triple crossover complexes. J Am Chem Soc 122, 1848-1860, (2000).

24 He, Y., Chen, Y., Liu, H., Ribbe, A. E. \& Mao, C. Self-assembly of hexagonal DNA two-dimensional (2D) arrays. J Am Chem Soc 127, 1220212203, (2005).

25 Douglas, S. M. et al. Rapid prototyping of 3D DNA-origami shapes with caDNAno. Nucleic Acids Res 37, 5001-5006, (2009). 
26 Yurke, B., Turberfield, A. J., Mills, A. P., Simmel, F. C. \& Neumann, J. L. A DNA-fuelled molecular machine made of DNA. Nature 406, 605-608, (2000).

27 Zhang, D. Y. \& Seelig, G. Dynamic DNA nanotechnology using stranddisplacement reactions. Nat Chem 3, 103-113, (2011).

28 Srinivas, N. et al. On the biophysics and kinetics of toehold-mediated DNA strand displacement. Nucleic Acids Res 41, 10641-10658, (2013).

29 SantaLucia, J. A unified view of polymer, dumbbell, and oligonucleotide DNA nearest-neighbor thermodynamics. P Natl Acad Sci USA 95, 14601465, (1998).

30 Zhang, D. Y. \& Winfree, E. Control of DNA Strand Displacement Kinetics Using Toehold Exchange. J Am Chem Soc 131, 17303-17314, (2009).

31 Green, S. J., Lubrich, D. \& Turberfield, A. J. DNA hairpins: Fuel for autonomous DNA devices. Biophys J 91, 2966-2975, (2006).

32 Zhang, D. Y. Cooperative Hybridization of Oligonucleotides. J Am Chem Soc 133, 1077-1086, (2011).

33 Genot, A. J., Zhang, D. Y., Bath, J. \& Turberfield, A. J. Remote Toehold: A Mechanism for Flexible Control of DNA Hybridization Kinetics. J Am Chem Soc 133, 2177-2182, (2011).

34 Zhang, D. Y. \& Winfree, E. Dynamic allosteric control of noncovalent DNA catalysis reactions. J Am Chem Soc 130, 13921-13926, (2008).

35 Yang, X. L., Tang, Y. N., Traynor, S. M. \& Li, F. Regulation of DNA Strand Displacement Using an Allosteric DNA Toehold. J Am Chem Soc 138, 14076-14082, (2016).

36 Jiang, Y. S., Bhadra, S., Li, B. L. \& Ellington, A. D. Mismatches Improve the Performance of Strand-Displacement Nucleic Acid Circuits. Angew Chem Int Edit 53, 1845-1848, (2014). 
37 Yin, P., Choi, H. M., Calvert, C. R. \& Pierce, N. A. Programming biomolecular self-assembly pathways. Nature 451, 318-322, (2008).

38 Thachuk, C., Winfree, E. \& Soloveichik, D. in International Workshop on DNA-Based Computers. 133-153 (Springer).

39 Zuker, M. Mfold web server for nucleic acid folding and hybridization prediction. Nucleic Acids Res 31, 3406-3415, (2003).

40 Zadeh, J. N. et al. NUPACK: Analysis and Design of Nucleic Acid Systems. Journal of computational chemistry 32, 170-173, (2011).

41 Doye, J. P. K. et al. Coarse-graining DNA for simulations of DNA nanotechnology. Phys Chem Chem Phys 15, 20395-20414, (2013).

42 Phillips, A. \& Cardelli, L. A programming language for composable DNA circuits. J R Soc Interface 6, (2009).

43 Lakin, M. R., Youssef, S., Polo, F., Emmott, S. \& Phillips, A. Visual DSD: a design and analysis tool for DNA strand displacement systems. Bioinformatics 27, 3211-3213, (2011).

44 Castro, C. E. et al. A primer to scaffolded DNA origami. Nat Methods 8, 221-229, (2011).

45 Williams, S. et al. Tiamat: A Three-Dimensional Editing Tool for Complex DNA Structures. Lect Notes Comput Sc 5347, 90-+, (2009).

46 Wei, B., Wang, Z. Y. \& Mi, Y. L. Uniquimer: Software of de novo DNA sequence generation for DNA self-assembly - An introduction and the related applications in DNA self-assembly. J Comput Theor Nanos 4, 133141, (2007).

47 Andersen, E. S. et al. DNA origami design of dolphin-shaped structures with flexible tails. Acs Nano 2, 1213-1218, (2008).

48 Seeman, N. C. An overview of structural DNA Nanotechnology. Mol Biotechnol 37, 246-257, (2007). 
49 Bath, J. \& Turberfield, A. J. DNA Nanomachines. Nature nanotechnology 2, 275-284, (2007).

50 Simmel, F. C. DNA-based assembly lines and nanofactories. Curr Opin Biotech 23, 516-521, (2012).

51 Torring, T., Voigt, N. V., Nangreave, J., Yan, H. \& Gothelf, K. V. DNA origami: a quantum leap for self-assembly of complex structures. Chem Soc Rev 40, 5636-5646, (2011).

52 Zhang, F., Nangreave, J., Liu, Y. \& Yan, H. Structural DNA nanotechnology: state of the art and future perspective. J Am Chem Soc 136, 11198-11211, (2014).

53 Tintore, M., Eritja, R. \& Fabrega, C. DNA nanoarchitectures: steps towards biological applications. Chembiochem 15, 1374-1390, (2014).

54 Zahid, M., Kim, B., Hussain, R., Amin, R. \& Park, S. H. DNA nanotechnology: a future perspective. Nanoscale research letters 8, 119, (2013).

55 Howorka, S. DNA Nanoarchitectonics: Assembled DNA at Interfaces. Langmuir, (2013).

56 Gothelf, K. V. Materials science. LEGO-like DNA structures. Science 338, 1159-1160, (2012).

57 Modi, S., Bhatia, D., Simmel, F. C. \& Krishnan, Y. Structural DNA Nanotechnology: From Bases to Bricks, From Structure to Function. J Phys Chem Lett 1, 1994-2005, (2010).

58 Liedl, T., Sobey, T. L. \& Simmel, F. C. DNA-based nanodevices. Nano Today 2, 36-41, (2007).

59 Seelig, G., Soloveichik, D., Zhang, D. Y. \& Winfree, E. Enzyme-free nucleic acid logic circuits. Science 314, 1585-1588, (2006). 
60 Li, B. L., Ellington, A. D. \& Chen, X. Rational, modular adaptation of enzyme-free DNA circuits to multiple detection methods. Nucleic Acids Res 39, (2011).

61 Zhang, D. Y., Turberfield, A. J., Yurke, B. \& Winfree, E. Engineering entropy-driven reactions and networks catalyzed by DNA. Science 318, 1121-1125, (2007).

62 Chen, X., Briggs, N., McLain, J. R. \& Ellington, A. D. Stacking nonenzymatic circuits for high signal gain. $P$ Natl Acad Sci USA 110, 53865391, (2013).

63 Yin, P., Choi, H. M. T., Calvert, C. R. \& Pierce, N. A. Programming biomolecular self-assembly pathways. Nature 451, 318-322, (2008).

64 Wickham, S. F. J. et al. A DNA-based molecular motor that can navigate a network of tracks. Nat Nanotechnol 7, 169-173, (2012).

65 Liu, M. H. et al. A DNA tweezer-actuated enzyme nanoreactor. Nat Commun 4, (2013).

66 Modi, S. et al. A DNA nanomachine that maps spatial and temporal $\mathrm{pH}$ changes inside living cells. Nature nanotechnology 4, 325-330, (2009).

67 Wang, Z. G., Elbaz, J., Remacle, F., Levine, R. D. \& Willner, I. All-DNA finite-state automata with finite memory. P Natl Acad Sci USA 107, 2199622001, (2010).

68 Sherman, W. B. \& Seeman, N. C. A precisely controlled DNA biped walking device. Nano Lett 4, 1203-1207, (2004).

69 Shin, J. S. \& Pierce, N. A. A synthetic DNA walker for molecular transport. J Am Chem Soc 126, 10834-10835, (2004).

70 Yin, P., Yan, H., Daniell, X. G., Turberfield, A. J. \& Reif, J. H. A unidirectional DNA walker that moves autonomously along a track. Angew Chem Int Edit 43, 4906-4911, (2004). 
71 Bath, J., Green, S. J. \& Turberfield, A. J. A free-running DNA motor powered by a nicking enzyme. Angew Chem Int Edit 44, 4358-4361, (2005).

72 Tian, Y., He, Y., Chen, Y., Yin, P. \& Mao, C. A DNAzyme that walks processively and autonomously along a one-dimensional track. Angewandte Chemie 44, 4355-4358, (2005).

73 Pei, R. et al. Behavior of polycatalytic assemblies in a substrate-displaying matrix. J Am Chem Soc 128, 12693-12699, (2006).

74 Green, S. J., Bath, J. \& Turberfield, A. J. Coordinated Chemomechanical Cycles: A Mechanism for Autonomous Molecular Motion. Phys Rev Lett 101, (2008).

75 Bath, J., Green, S. J., Allen, K. E. \& Turberfield, A. J. Mechanism for a Directional, Processive, and Reversible DNA Motor. Small 5, 1513-1516, (2009).

76 Omabegho, T., Sha, R. \& Seeman, N. C. A Bipedal DNA Brownian Motor with Coordinated Legs. Science 324, 67-71, (2009).

$77 \mathrm{Gu}$, H. Z., Chao, J., Xiao, S. J. \& Seeman, N. C. A proximity-based programmable DNA nanoscale assembly line. Nature 465, 202-U286, (2010).

78 He, Y. \& Liu, D. R. Autonomous multistep organic synthesis in a single isothermal solution mediated by a DNA walker. Nature nanotechnology 5, 778-782, (2010).

79 Lund, K. et al. Molecular robots guided by prescriptive landscapes. Nature 465, 206-210, (2010).

80 Muscat, R. A., Bath, J. \& Turberfield, A. J. A Programmable Molecular Robot. Nano letters 11, 982-987, (2011).

81 Wang, C. Y., Ren, J. S. \& Qu, X. G. A stimuli responsive DNA walking device. Chem Commun 47, 1428-1430, (2011). 
82 Wang, Z. G., Elbaz, J. \& Willner, I. DNA Machines: Bipedal Walker and Stepper. Nano Lett 11, 304-309, (2011).

83 Wickham, S. F. J. et al. Direct observation of stepwise movement of a synthetic molecular transporter. Nature nanotechnology 6, 166-169, (2011).

84 Cheng, J. et al. Bipedal Nanowalker by Pure Physical Mechanisms. Phys Rev Lett 109, (2012).

85 Masoud, R. et al. Studying the Structural Dynamics of Bipedal DNA Motors with Single-Molecule Fluorescence Spectroscopy. Acs Nano 6, 6272-6283, (2012).

86 Muscat, R. A., Bath, J. \& Turberfield, A. J. Small Molecule Signals that Direct the Route of a Molecular Cargo. Small 8, 3593-3597, (2012).

87 Wang, C. Y., Tao, Y., Song, G. T., Ren, J. S. \& Qu, X. G. Speeding Up a Bidirectional DNA Walking Device. Langmuir 28, 14829-14837, (2012).

88 You, M. X. et al. An Autonomous and Controllable Light-Driven DNA Walking Device. Angew Chem Int Edit 51, 2457-2460, (2012).

89 You, M. X., Huang, F. J., Chen, Z., Wang, R. W. \& Tan, W. H. Building a Nanostructure with Reversible Motions Using Photonic Energy. Acs Nano 6, 7935-7941, (2012).

90 Tomov, T. E. et al. Rational Design of DNA Motors: Fuel Optimization through Single-Molecule Fluorescence. J Am Chem Soc 135, 11935-11941, (2013).

91 Cha, T. G. et al. A synthetic DNA motor that transports nanoparticles along carbon nanotubes. Nature nanotechnology 9, 39-43, (2014).

92 Liu, M. H. et al. Autonomous Synergic Control of Nanomotors. Acs Nano 8, 1792-1803, (2014).

93 Loh, I. Y., Cheng, J., Tee, S. R., Efremov, A. \& Wang, Z. S. From Bistate Molecular Switches to Self-Directed Track-Walking Nanomotors. Acs Nano 8, 10293-10304, (2014). 
94 Liber, M., Tomov, T. E., Tsukanov, R., Berger, Y. \& Nir, E. A Bipedal DNA Motor that Travels Back and Forth between Two DNA Origami Tiles. Small 11, 568-575, (2015).

95 Jung, C., Allen, P. B. \& Ellington, A. D. A stochastic DNA walker that traverses a microparticle surface. Nature nanotechnology 11, 157-163, (2016).

96 Chen, Y. J. et al. Programmable chemical controllers made from DNA. Nature nanotechnology 8, 755-762, (2013).

97 Qian, L., Winfree, E. \& Bruck, J. Neural network computation with DNA strand displacement cascades. Nature 475, 368-372, (2011).

98 Chandran, H., Gopalkrishnan, N., Yurke, B. \& Reif, J. Meta-DNA: synthetic biology via DNA nanostructures and hybridization reactions. $J R$ Soc Interface 9, 1637-1653, (2012).

99 Turberfield, A. J. et al. DNA fuel for free-running nanomachines. Phys Rev Lett 90, (2003).

100 Dirks, R. M. \& Pierce, N. A. Triggered amplification by hybridization chain reaction. Proc. Natl. Acad. Sci. U. S. A. 101, 15275-15278, (2004).

101 Lubrich, D., Green, S. J. \& Turberfield, A. J. Kinetically Controlled SelfAssembly of DNA Oligomers. J Am Chem Soc 131, 2422-+, (2009).

102 Venkataraman, S., Dirks, R. M., Rothemund, P. W. K., Winfree, E. \& Pierce, N. A. An autonomous polymerization motor powered by DNA hybridization. Nature nanotechnology 2, 490-494, (2007).

103 Li, B., Jiang, Y., Chen, X. \& Ellington, A. D. Probing spatial organization of DNA strands using enzyme-free hairpin assembly circuits. J Am Chem Soc 134, 13918-13921, (2012).

104 Chandran, H., Gopalkrishnan, N., Phillips, A. \& Reif, J. in DNA Computing and Molecular Programming Vol. 6937 Lecture Notes in Computer Science (eds L. Cardelli \& W. Shih) 64-83 (Springer Berlin Heidelberg, 2011). 
105 Teichmann, M., Kopperger, E. \& Simmel, F. C. Robustness of Localized DNA Strand Displacement Cascades. Acs Nano, (2014).

106 Garg, S. Programming Molecular Devices using Nucleic Acid Hairpins Ph.D. thesis, Duke University, (2016).

107 IDTDNA. DNA Thermodynamics and Hybridization, $<$ http://biophysics.idtdna.com> (2017).

108 Dirks, R. M. \& Pierce, N. A. A partition function algorithm for nucleic acid secondary structure including pseudoknots. J Comput Chem 24, 1664-1677, (2003).

109 Yurke, B. \& Mills, A. P. Using DNA to Power Nanostructures. Genetic Programming and Evolvable Machines 4, 111-122, (2003).

110 Dalchau, N., Chandran, H., Gopalkrishnan, N., Phillips, A. \& Reif, J. Probabilistic analysis of localized DNA hybridization circuits. ACS Synth Biol, (2015).

111 Elezgaray, J. et al. Connecting Localized DNA Strand Displacement Reactions. Nanoscale, (2015).

112 Kopperger, E., Pirzer, T. \& Simmel, F. C. Diffusive transport of molecular cargo tethered to a DNA origami platform. Nano Lett 15, 2693-2699, (2015).

113 Muscat, R. A., Strauss, K., Ceze, L. \& Seelig, G. in ISCA '13: Proceedings of the 40th Annual International Symposium on Computer Architecture Vol. 41 (ed Avi Mendelson) 177-188 (ACM, 2013).

114 Seelig, G., Yurke, B. \& Winfree, E. in Lect Notes Comput Sc Vol. 3384 Theoretical Computer Science and General Issues (eds Claudio Ferretti, Giancarlo Mauri, \& Claudio Zandron) 329-343 (Springer Berlin Heidelberg, 2005).

115 Soloveichik, D., Seelig, G. \& Winfree, E. DNA as a Universal Substrate for Chemical Kinetics. Proc. Natl. Acad. Sci. U. S. A. 107, 5393-5398, (2010). 
116 Zhang, D. Y., Chen, S. X. \& Yin, P. Optimizing the specificity of nucleic acid hybridization. Nat Chem 4, 208-214, (2012).

117 Zhang, D. Y. \& Winfree, E. Robustness and modularity properties of a non-covalent DNA catalytic reaction. Nucleic Acids Res 38, 4182-4197, (2010).

118 Dannenberg, F., Kwiatkowska, M., Thachuk, C. \& Turberfield, A. in Lect Notes Comput Sc Vol. 8141 Lecture Notes in Computer Science (eds David Soloveichik \& Bernard Yurke) Ch. 3, 31-45 (Springer International Publishing, 2013).

119 Teichmann, M., Kopperger, E. \& Simmel, F. C. Robustness of localized DNA strand displacement cascades. Acs Nano 8, 8487-8496, (2014).

120 Ruiz, I. M. et al. Connecting localized DNA strand displacement reactions. Nanoscale 7, 12970-12978, (2015).

121 Dunn, K. E., Trefzer, M. A., Johnson, S. \& Tyrrell, A. M. Investigating the dynamics of surface-immobilized DNA nanomachines. Sci Rep-Uk 6, (2016).

122 Choi, H. M. T. et al. Programmable in situ amplification for multiplexed imaging of mRNA expression. Nat Biotechnol 28, 1208-1212, (2010).

123 Meng, W. J. et al. An autonomous molecular assembler for programmable chemical synthesis. Nat Chem 8, 542-548, (2016).

124 Sadowski, J. P., Calvert, C. R., Zhang, D. Y., Pierce, N. A. \& Yin, P. Developmental Self-Assembly of a DNA Tetrahedron. Acs Nano 8, 32513259, (2014).

125 Pinheiro, A. V., Han, D. R., Shih, W. M. \& Yan, H. Challenges and opportunities for structural DNA nanotechnology. Nature nanotechnology 6, 763-772, (2011).

126 Chao, J. et al. Structural DNA Nanotechnology for Intelligent Drug Delivery. Small, (2014). 
127 Khodakov, D., Wang, C. \& Zhang, D. Y. Diagnostics based on nucleic acid sequence variant profiling: PCR, hybridization, and NGS approaches. Advanced drug delivery reviews 105, 3-19, (2016).

128 Keum, J. W. \& Bermudez, H. Enhanced resistance of DNA nanostructures to enzymatic digestion. Chem Commun (Camb), 7036-7038, (2009).

129 Mei, Q. et al. Stability of DNA origami nanoarrays in cell lysate. Nano Lett 11, 1477-1482, (2011).

130 Conway, J. W., McLaughlin, C. K., Castor, K. J. \& Sleiman, H. DNA nanostructure serum stability: greater than the sum of its parts. Chem Commun (Camb) 49, 1172-1174, (2013).

131 Lee, H. et al. Molecularly self-assembled nucleic acid nanoparticles for targeted in vivo siRNA delivery. Nature nanotechnology 7, 389-393, (2012).

132 Charoenphol, P. \& Bermudez, H. Aptamer-Targeted DNA Nanostructures for Therapeutic Delivery. Mol Pharmaceut 11, 1721-1725, (2014).

133 Liu, X. W. et al. A DNA Nanostructure Platform for Directed Assembly of Synthetic Vaccines. Nano Lett 12, 4254-4259, (2012).

134 Jiang, Q. et al. DNA Origami as a Carrier for Circumvention of Drug Resistance. J Am Chem Soc 134, 13396-13403, (2012).

135 Dalchau, N., Chandran, H., Gopalkrishnan, N., Phillips, A. \& Reif, J. Probabilistic Analysis of Localized DNA Hybridization Circuits. ACS synthetic biology 4, 898-913, (2015).

136 Sharonov, A. \& Hochstrasser, R. M. Wide-field subdiffraction imaging by accumulated binding of diffusing probes. Proc. Natl. Acad. Sci. U. S. A. 103, 18911-18916, (2006).

137 Jungmann, R. et al. Single-Molecule Kinetics and Super-Resolution Microscopy by Fluorescence Imaging of Transient Binding on DNA Origami. Nano Lett 10, 4756-4761, (2010). 
138 Jungmann, R. et al. Quantitative super-resolution imaging with qPAINT. Nat Methods 13, 439-442, (2016).

139 Park, S. H. et al. Three-helix bundle DNA tiles self-assemble into 2D lattice or 1D templates for silver nanowires. Nano letters 5, 693-696, (2005).

140 Reif, J. H. DNA lattices: A method for molecular-scale patterning and computation. Comput Sci Eng 4, 32-41, (2002).

141 Torring, T., Voigt, N. V., Nangreave, J., Yan, H. \& Gothelf, K. V. DNA origami: a quantum leap for self-assembly of complex structures. Chem Soc Rev 40, 5636-5646, (2011).

142 Sacca, B. \& Niemeyer, C. M. DNA Origami: The Art of Folding DNA. Angew Chem Int Edit 51, 58-66, (2012).

143 Jungmann, R. et al. Multiplexed 3D cellular super-resolution imaging with DNA-PAINT and Exchange-PAINT. Nat Methods 11, 313-318, (2014).

144 Hell, S. W. \& Wichmann, J. Breaking the Diffraction Resolution Limit by Stimulated-Emission - Stimulated-Emission-Depletion Fluorescence Microscopy. Opt Lett 19, 780-782, (1994).

145 Rust, M. J., Bates, M. \& Zhuang, X. W. Sub-diffraction-limit imaging by stochastic optical reconstruction microscopy (STORM). Nat Methods 3, 793795, (2006).

146 Hess, S. T., Girirajan, T. P. K. \& Mason, M. D. Ultra-high resolution imaging by fluorescence photoactivation localization microscopy. Biophys J 91, 4258-4272, (2006).

147 Betzig, E. et al. Imaging intracellular fluorescent proteins at nanometer resolution. Science 313, 1642-1645, (2006).

148 Folling, J. et al. Fluorescence nanoscopy by ground-state depletion and single-molecule return. Nat Methods 5, 943-945, (2008). 
149 Kiuchi, T., Higuchi, M., Takamura, A., Maruoka, M. \& Watanabe, N. Multitarget super-resolution microscopy with high-density labeling by exchangeable probes. Nat Methods 12, 743-U769, (2015).

150 Lin, C. X. et al. Submicrometre geometrically encoded fluorescent barcodes self-assembled from DNA. Nat Chem 4, 832-839, (2012).

151 Reif, J. H. Parallel biomolecular computation: Models and simulations. Algorithmica 25, 142-175, (1999).

152 Mao, C. D., LaBean, T. H., Reif, J. H. \& Seeman, N. Logical computation using algorithmic self-assembly of DNA triple-crossover molecules (vol 407, pg 493, 2000). Nature 408, 750-750, (2000).

153 Yan, H., Feng, L. P., LaBean, T. H. \& Reif, J. H. Parallel molecular computations of pairwise exclusive or (XOR) using DNA "String tile" selfassembly. J Am Chem Soc 125, 14246-14247, (2003).

154 Yin, P., Sahu, S., Turberfield, A. J. \& Reif, J. H. Design of autonomous DNA cellular automata. Lect Notes Comput Sc 3892, 399-416, (2006).

155 Majumder, U. \& Reif, J. H. Design of a Biomolecular Device That Executes Process Algebra. Lect Notes Comput Sc 5877, 97-105, (2009).

156 Reif, J. H. The design of autonomous DNA nanomechanical devices: Walking and rolling DNA. Lect Notes Comput Sc 2568, 22-37, (2003).

157 Reif, J. H., LaBean, T. H., Sahu, S., Yan, H. \& Yin, P. Design, simulation, and experimental demonstration of self-assembled DNA nanostructures and motors. Lect Notes Comput Sc 3566, 173-187, (2005).

158 Yin, P., Turberfield, A. J. \& Reif, J. H. Designs of autonomous unidirectional walking DNA devices. Lect Notes Comput Sc 3384, 410-425, (2005).

159 Yin, P., Turberfield, A. J., Sahu, S. \& Reif, J. H. Design of an autonomous DNA nanomechanical device capable of universal computation and universal translational motion. Lect Notes Comput Sc 3384, 426-444, (2005). 
160 Sahu, S., LaBean, T. H. \& Reif, J. H. A DNA Nanotransport Device Powered by Polymerase phi 29. Nano letters 8, 3870-3878, (2008).

161 Feng, L. P., Park, S. H., Reif, J. H. \& Yan, H. A two-state DNA lattice switched by DNA nanoactuator. Angew Chem Int Edit 42, 4342-4346, (2003).

162 Reif, J. H. \& Sahu, S. Autonomous programmable nanorobotic devices using DNAzymes. Lect Notes Comput Sc 4848, 66-78, (2008).

163 Chandran, H., Gopalkrishnan, N., Phillips, A. \& Reif, J. Localized Hybridization Circuits. Lect Notes Comput Sc 6937, 64-83, (2011).

164 Bui, H. et al. in Computing Handbook Vol. Volume I: Computer Science and Software Engineering (ed Teofilo Gonzalez) (Taylor \& Francis Group, 2013).

165 Bui, H. et al. Design and analysis of linear cascade DNA hybridization chain reactions using DNA hairpins. New Journal of Physics 19, 015006, (2017).

166 Bui, H. et al. Localized DNA Hybridization Chain Reactions on DNA Origami. (submitted).

167 Greenberg, J. M. \& Hastings, S. Spatial patterns for discrete models of diffusion in excitable media. SIAM Journal on Applied Mathematics 34, 515523, (1978).

168 Myers, N. Rendering life molecular: models, modelers, and excitable matter. (Duke University Press, 2015).

169 Letichevskii, A. \& Reshod'ko, L. N. Wiener's theory of the activity of excitable media. Cybernetics and Systems Analysis 8, 856-864, (1972).

170 Moe, G. K., Rheinboldt, W. C. \& Abildskov, J. A computer model of atrial fibrillation. American heart journal 67, 200-220, (1964).

171 Wiener, N. \& Rosenblueth, A. The mathematical formulation of the problem of conduction of impulses in a network of connected excitable 
elements, specifically in cardiac muscle. Archivos del instituto de Cardiología de México 16, 205-265, (1946).

172 Fisch, R., Gravner, J. \& Griffeath, D. Metastability in the GreenbergHastings model. The Annals of Applied Probability, 935-967, (1993).

173 Durrett, R. \& Steif, J. E. Some rigorous results for the Greenberg-Hastings model. Journal of Theoretical Probability 4, 669-690, (1991).

174 Gerhardt, M., Schuster, H. \& Tyson, J. J. A cellular automaton model of excitable media: Ii. curvature, dispersion, rotating waves and meandering waves. Physica D: Nonlinear Phenomena 46, 392-415, (1990).

175 McCulloch, W. S. \& Pitts, W. A logical calculus of the ideas immanent in nervous activity. The bulletin of mathematical biophysics 5, 115-133, (1943).

176 Maan, A. K., Jayadevi, D. A. \& James, A. P. A Survey of Memristive Threshold Logic Circuits. (2016).

177 Anthony, M. Discrete mathematics of neural networks: selected topics. Vol. 8 (Siam, 2001).

178 Dayan, P. \& Abbott, L. Theoretical neuroscience: computational and mathematical modeling of neural systems. Journal of Cognitive Neuroscience 15, 154-155, (2003).

179 Joshi, J., Hsu, C., Parker, A. C. \& Deshmukh, P. in IEEE/NIH LIfe Science Systems and Applications Workshop.

180 Joshi, J., Parker, A. C. \& Hsu, C.-C. in 2009 Annual International Conference of the IEEE Engineering in Medicine and Biology Society. 1651-1654 (IEEE).

181 Parker, A. C., Joshi, J., Hsu, C.-C. \& Singh, N. A. D. in 2008 51st Midwest Symposium on Circuits and Systems. 818-821 (IEEE).

182 Joshi, J. et al. in 2011 IEEE/NIH Life Science Systems and Applications Workshop (LiSSA).

183 Mead, C. Neuromorphic electronic systems. Proceedings of the IEEE 78, 1629-1636, (1990). 
184 Monroe, D. Neuromorphic computing gets ready for the (really) big time. Communications of the ACM 57, 13-15, (2014).

185 Douglas, S. M., Bachelet, I. \& Church, G. M. A Logic-Gated Nanorobot for Targeted Transport of Molecular Payloads. Science 335, 831-834, (2012).

186 Fu, J. L. \& Yan, H. Controlled drug release by a nanorobot. Nat Biotechnol 30, 407-408, (2012).

187 Langecker, M. et al. Synthetic Lipid Membrane Channels Formed by Designed DNA Nanostructures. Science 338, 932-936, (2012). 


\section{Biography}

Hieu Trung Bui (March 19 ${ }^{\text {th }}$ 1985) was born in Saigon, Vietnam. He received B.S. and M.S. in Electrical and Computer Engineering from Boise State University in 2008 and 2011, respectively. He received his Ph.D. in Computer Science from Duke University in May 2017. He is a recipient of Micron scholarship, Duke Graduate School Fellowship, and Duke GPNano Fellowship. He presented his research at Foundations of Nanoscience Conference (2008, 2009, 2010, 2012, 2013, 2014, 2015, 2016) and International Conference on DNA Computing and Molecular Programming (DNA 19, DNA 20, DNA 21, DNA 22). He is a member

of NSCS, Eta Kappa Nu, ISNSCE. He has published papers and book chapters to Nano Letters, ACS Synthetic Biology, Small, and New Journal of Physics.

1. Bui, H.; Miao, V.; Garg, S.; Mokhtar, R.; Song, T.; Reif, J., Design and Analysis of Localized DNA Hybridization Chain Reactions. Small 2017, 1602983.

2. Bui, H.; Garg, S.; Miao, V.; Song, T.; Mohktar, R.; Reif, J., Design and Analysis of Linear Cascade DNA Hybridization Chain Reactions Using DNA Hairpins. New Journal of Physics 2017, 19 (1), 015006.

3. Song, T. Q.; Garg, S.; Mokhtar, R.; Bui, H.; Reif, J., Analog Computation by DNA Strand Displacement Circuits. ACS Synth. Biol. 2016, 5 (8), 898-912.

4. Mokhtar, R.; Garg, S.; Chandran, H.; Bui, H.; Song, T.; Reif, J., Modeling DNA Nanodevices Using Graph Rewriting Systems. In Advanced in Unconventional Computing, Adamatzky, A., Ed. Springer: 2015; Vol. Prototypes, Models and Algorithms, pp 347-396. 
5. Bui, H.; Chandran, H.; Garg, S.; Gopalkrishnan, N.; Mokhtar, R.; Song, T.; Reif, J. H., DNA Computing. In Computing Handbook, Gonzalez, T., Ed. Taylor \& Francis Group: 2013; Vol. Volume I: Computer Science and Software Engineering.

6. Graugnard, E.; Kellis, D. L.; Bui, H.; Barnes, S.; Kuang, W.; Lee, J.; Hughes, W. L.; Knowlton, W. B.; Yurke, B., DNA-controlled excitonic switches. Nano Lett 2012, $12(4), 2117-22$.

7. Bui, H.; Onodera, C.; Kidwell, C.; Tan, Y.; Graugnard, E.; Kuang, W.; Lee, J.; Knowlton, W. B.; Yurke, B.; Hughes, W. L., Programmable periodicity of quantum dot arrays with DNA origami nanotubes. Nano Lett 2010, 10 (9), 3367-72. 